This item was submitted to Loughborough's Research Repository by the author.

Items in Figshare are protected by copyright, with all rights reserved, unless otherwise indicated.

\title{
Graphene materials in green energy applications: Recent development and future perspective
}

\section{PLEASE CITE THE PUBLISHED VERSION}

https://doi.org/10.1016/j.rser.2019.109656

\section{PUBLISHER}

Elsevier

\section{VERSION}

AM (Accepted Manuscript)

\section{PUBLISHER STATEMENT}

This paper was accepted for publication in the journal Renewable and Sustainable Energy Reviews and the definitive published version is available at https://doi.org/10.1016/j.rser.2019.109656

\section{LICENCE}

CC BY-NC-ND 4.0

\section{REPOSITORY RECORD}

Tsang, Chi Him Alpha, Haibao Huang, Jin Xuan, Huizhi Wang, and DYC Leung. 2019. "Graphene Materials in Green Energy Applications: Recent Development and Future Perspective”. Loughborough University. https://hdl.handle.net/2134/14046716.v1. 


\title{
Graphene Materials in Green Energy Applications: Recent Development and Future Perspective
}

\author{
Chi Him Alpha Tsang ${ }^{a, b}$, Haibao Huang ${ }^{a}$, Jin Xuan ${ }^{c^{*}}$, Huizhi Wang ${ }^{d^{*}}$, DYC Leung ${ }^{b^{*}}$ \\ ${ }^{a}$ School of Environmental Science and Engineering, Sun Yat-Sen University, Guangzhou, \\ PRC \\ ${ }^{\mathrm{b}}$ Department of Mechanical Engineering, The University of Hong Kong, Hong Kong \\ ${ }^{c}$ Department of Chemical Engineering, Loughborough University, Loughborough, UK \\ ${ }^{\mathrm{d}}$ Department of Mechanical Engineering, Imperial College London, London, UK \\ * Corresponding authors, ycleung@hku.hk (DYCL), j.xuan@1boro.ac.uk (JX), \\ huizhi.wang@imperial.ac.uk (HW)
}

\begin{abstract}
Graphene, composed of single-layered $\mathrm{sp}^{2}$ graphite, with its superb physical and chemical properties, has attracted scientists in different areas, including electronics, medicine, and chemicals. Its applications in green energy were extensively studied in the past two decades. Results from different studies show that graphene-based products with different structures (2D and 3D graphene) can effectively enhance green energy conversion and storage compared to traditional energy materials like metal and metal oxides. This review focuses on the historical development of graphene, the variation of graphene products, and mainstream researches in graphene-based green energy applications carried out in recent years, such as fuel cell, solar cell, lithium-ion batteries (LIBs), supercapacitor, dye-sensitized solar cell (DSSC), and photoelectrochemical water-splitting cell. The development of graphene-based materials integrated with different 3D printing technology and the application of their products in green energy are also discussed together with a forecast on the development of graphene-based materials in the future.
\end{abstract}

\section{Highlight:}

1. Development of raw graphene (bottom-up and top-down) is highlighted. 
2. Various modifications of graphene-based materials for energy applications are reviewed.

3. Use of graphene-based materials in green energy electrodes is discussed.

4. Recent development of graphene in green energy via 3D printing is introduced.

5. Perspective on graphene energy materials development is given.

Keyword: Graphene, fuel cell, supercapacitor, battery, solar cell, 3D printing

Word count: 9253 words

List of Abbreviations:

LIBs: Lithium-ion batteries

DSSC: Dye-sensitized solar cells

CVD: Chemical vapor deposition

GO: Graphene oxide

LPE: Liquid phase exfoliation

NMP: N-methylpyrrolidone

DMF: N,N'-dimethylformamide

DMSO: Dimethyl sulfoxide

2D: Two dimensional

3D: Three dimensional

HR-XPS: High resolution-X-Ray photoelectron spectroscopy

rGO, RGO, G, GNPs: Reduced graphene oxide (nanoplates)

GH: Graphene hydrogel

GSA: Geometric Surface Area

EGS: Expandable graphene sheets 
VC: L-ascorbic acid/vitamin C

GA: Graphene aerogel

NF: Ni foam

PNWs: PVP-metal nanowires hybrid

G-Gel/NF, G-gel@NF: Graphene hydrogel/Ni foam

M/GN: Metal modified graphene

$\mathrm{MO}_{\mathrm{x}} / \mathrm{GN}$ : Metal oxide modified graphene

EG: Ethyl glycol

PD: 1,2-propanediol

IPA: Isopropyl alcohol

Nf: Nafion solution

NCs: Nanocrystal

NGA, N-GA: N-doped graphene aerogel

GN-GH: nitrogen doped graphene hydrogel

NG/NF: nitrogen-doped graphene on nickel foam

a-MEGO: activated microwave exfoliated graphite oxide

aG-O film: activated graphene oxide film

NSGAs: nitrogen and sulfur dual-doped graphene aerogels

$\alpha-\mathrm{MnO}_{2} / \mathrm{RGO}: \mathrm{MnO}_{2}$ nanowires/Reduced graphene oxide

3DE/Au: gold loaded three-dimensional printed electrode

$\mathrm{Ru} / \mathrm{r}-\mathrm{hGO}$ mesh: ruthenium loaded reduced 3D printed holey graphene oxide mesh

$3 \mathrm{D} \mathrm{G} / \mathrm{MnO}_{2}: 3 \mathrm{D}$ printed graphene aerogel/ $\mathrm{MnO}_{2}$

B-G: Boron doped graphene

$\mathrm{N}$-doped graphene deposited on nickel foam: Nitrogen-doped graphene deposited on nickel foam 
BT-rGO: B-doped graphene nanoplatelets

$\mathrm{BCN}$ graphene: Boron and nitrogen co-doped graphene

SP-AG: 3D macroporous, activated graphene aerogel

NP: Nanoparticle

NF(s) with Metal (M) prefix: Metal Nanoflower(s)

CFP: Carbon fibre paper

GF: Graphene foam or 3D graphene frame work

GQD: Graphene quantum dot

GA@NF: graphene aerogels on NF

HrGO/NF: hydrothermal reduced graphene oxide/nickel foam

3D-NiGO(M): 3D Ni foam-supported graphene oxide (support for $\mathrm{MnO}_{2}$ )

GCA@NF: graphene/CNT hybrid aerogels on NF

rGH: reduced graphene hydrogel

RGOA: reduced graphene oxide aerogel

GO-RFA-P: pyrolyzed GO-loaded RF (resorcinol and formaldehyde) aerogels

SGH, SGHs: Self-assembled graphene hydrogel

GH-Hz8: Graphene hydrogels prepared via hydrothermal reduction with hydrazine (8 hours)

FGSs: Functionalized graphene sheets

FAG 400: 3D self-assembly of holey graphene (reduced at $400^{\circ} \mathrm{C}$ )

Graphene- $\mathrm{Fe}_{3} \mathrm{O}_{4} @ \mathrm{C}$ : Graphene-iron (IV) oxide on carbon

Graphene- $\mathrm{Co}_{3} \mathrm{O}_{4} @ \mathrm{C}$ : Graphene-cobalt (IV) oxide on carbon

$\mathrm{ZnO} / \mathrm{rGO}, \mathrm{ZnO} / \mathrm{RGO}$ : Zinc oxide nanosheet/reduced graphene oxide composite

ATN/RGO: anatase $\mathrm{TiO}_{2}$ nanosheet/reduced graphene oxide composite

3D CMG/MnO $2: 3 \mathrm{D}$ porous chemically modified graphene/ $\mathrm{MnO}_{2}$ foam

$\mathrm{GA} / \mathrm{TiO}_{2}$ : graphene aerogel/ $\mathrm{TiO}_{2}$ 
$\mathrm{Ni}(\mathrm{OH})_{2} \cdot(\mathrm{GN}): \mathrm{Ni}(\mathrm{OH})_{2}$ nanoparticles in graphene

GNS/LDH, graphene/NiAl-LDH: chemically converted graphene nanosheet/ $\mathrm{Ni}^{2+} / \mathrm{Al}^{3+}$ layered double-hydroxide (LDH) composite

3D-ARGON/NiAl-LDH: 3D activated reduced graphene oxide nanocup/nickel aluminum layered double hydroxides composite

GNS/CoAl-LDH: graphene nanosheet/CoAl-layered double hydroxide

Co-Al LDH-NS/GO: Co-Al layered double hydroxide nanosheets/GO

$\mathrm{Gr} / \mathrm{SnO}_{2}$ : graphene/tin dioxide nanoparticles composites

PANI@3DGFs: PANI loaded three-dimensional graphene-based frameworks

SRGA: S-incorporated RGO aerogel

STEM: Scanning Tunnel Electron Microscopy

GNFs: Graphene nanoflake films

ORR: Oxygen reduction reactions

MOR: Methanol oxidation reaction

EtOH: Ethanol

EOR: EtOH electrooxidation

GOR: Glucose electrooxidation

FOR: Formic acid electrooxidation

DEFC: Direct ethanol fuel cell

DGFC: Direct glucose fuel cell

HOR: Hydrogen oxidation reaction

Li-Air, Li-O ${ }_{2}$ : Lithium-air

HGF: Holey graphene framework

OCV: Open circuit voltage

ZGR: ZnO graphene 
GT: Graphene- $\mathrm{TiO}_{2}$

SLA: Stereolithography

FDM: Fused deposition modeling

DIW: Direct ink writing

IGF: integrated graphene network

MOG: $\mathrm{MnO}_{2}$ loaded 3D printed IGF

NOG: NiO loaded 3D printed IGF

PLA: Polylactic acid

PVA: Polyvinyl alcohol

PVB: Polyvinyl butyral

Nomenclature

$\mathrm{KMnO}_{4}$ : Potassium permanganate

$\mathrm{NaNO}_{3}$ : Sodium nitrate

$\mathrm{P}_{2} \mathrm{O}_{5}$ : Phosphorus pentoxide

$\mathrm{K}_{2} \mathrm{~S}_{2} \mathrm{O}_{8}$ : Potassium persulphate

$\mathrm{H}_{2} \mathrm{O}_{2}$ : Hydrogen peroxide

$\mathrm{CO}_{2}$ : Carbon dioxide

$\mathrm{C}=\mathrm{O}$ : Carbonyl group

$\mathrm{O}-\mathrm{C}=\mathrm{O}$ : Carboxyl group

C-O bonds: Ether link

Zn: Zinc

$\mathrm{NH}_{3}$ : Ammonia

$\mathrm{N}_{2} \mathrm{H}_{4}, \mathrm{~Hz}$ : Hydrazine

$\mathrm{BH}_{4}{ }^{-}$: Boron hydride species/borohydride 
$\mathrm{PtCl}_{6}{ }^{2-}:$ Hexachloroplatinate (IV)

$\mathrm{LiAlH}_{4}$ : Lithium aluminum hydride

CO: Carbon monoxide

PANI: polyaniline

Pd: Palladium

Pt: Platinum

$\mathrm{J}_{\mathrm{f}}$ : anodic sweep current density

LITFSI: Lithium bis(trifluoromethanesulfonyl) imide

$\mathrm{LiPF}_{6}$ : Lithium hexafluorophosphate

$\mathrm{Nb}_{2} \mathrm{O}_{5}$ : Niobia

S: Sulphur

P: Phosphorus

B, N-doped or BN-codoped: Boron-nitrogen codoped

$\mathrm{HClO}_{4}$ : Chloric (VII) acid

$\mathrm{H}_{2} \mathrm{SO}_{4}$ : Sulphuric Acid

$\mathrm{NaOH}$ : Sodium hydroxide

KOH: Potassium hydroxide

$\mathrm{Co}_{3} \mathrm{O}_{4}$ : Cobalt (IV) oxide

$\mathrm{J}_{\mathrm{sc}}$ : Short circuit Current

$\mathrm{TiO}_{2}$ : Titania or P25

Au: Gold

\section{Introduction}

Graphene is an allotrope of graphite composed of single-layered $\mathrm{sp}^{2}$ graphite with hexagonal planar carbon ring arrangement structures. It is a 2D nanosheet structured material, 
which is a derivative of $3 \mathrm{D}$ giant covalent structured graphite $[1,2]$. These materials have attractive properties of strong electrical conductivity $\left(10^{6} \mathrm{~S} \mathrm{~cm}^{-1}\right)$ [3], strong thermal conductivity (5000 $\left.\mathrm{W} \mathrm{m}^{-1} \mathrm{k}^{-1}\right)$ [4], high mechanical strength $\left(\sim 40 \mathrm{~N} \mathrm{~m}^{-1}\right)$, with Young's module of $1 \mathrm{TPa}$ [5], and large specific surface areas $\left(\sim 2600 \mathrm{~m}^{2} \mathrm{~g}^{-1}\right)$ [6]. Furthermore, its special chemical reactivity towards foreign materials compared to the bulk carbon like charcoal makes it not replaceable by other nanocarbons, such as carbon nanotubes and fullerenes. The development of graphene as new promising materials became solidified in the past decade, especially in the area of catalysis.

Due to the attractive properties mentioned above, a variety of graphene-based materials like metal, metal oxide, or non-metal element-doped graphene products were developed. They exist in $2 \mathrm{D}$ and $3 \mathrm{D}$ structures. However, most of the research on graphene-based products covered 2D structured graphene; and the 3D structured ones in bulk form were relatively fewer [7-9]. The import of $3 \mathrm{D}$ printing technology in recent years widens the development potential of 3D structured graphene for different applications [10-13].

There is a variety of research on graphene-based materials applied in green energy such as fuel cell, solar cell, lithium-ion batteries (LIBs), supercapacitor, dye-sensitized solar cells (DSSC), and photoelectrochemical cell for hydrogen evolution via water splitting. This type of research was conducted extensively in the past decade. Even though much effort has been devoted to it, most of the works were still in the fundamental laboratory scale. Only few real applications were reported [7-9]. Importing new technology for graphene fabrication (i.e. 3D printing) may provide a breakthrough in the limitation of traditional fabrication techniques for graphene-based device preparation [10-13].

This review is focused on the historical development of graphene and graphene products in the first part. The second part describes the mainstream researches on graphene-based materials applied in green energy, such as fuel cell, solar cell, LIBs, supercapacitor, DSSC, 
and photoelectrochemical cell. The recent development of graphene-based materials by integration with different 3D printing technologies and their applications in green energy were discussed in the third part followed by a forecast on the future development of graphene-based materials.

\section{History of raw graphene synthesis}

With the development in past decades, graphene synthesis has become a mature technology. There are two major pathways of graphene synthesis: 'Bottom-up' and 'Topdown' methods [14-17]. 'Bottom-up' is synthesis by depositing the carbon precursor vapor onto secondary substrate as seed for the growth of graphene, while the 'top-down' method is carried out through exfoliation by mechanical or chemical pathways to separate the carbon layer from the giant structure of graphite oxide, or through the knocking down of the graphite structure into a single graphene sheet as the final product [14-17]. In this review, syntheses of raw graphene via chemical vapor deposition (CVD) and chemical oxidation were chosen as representative synthesis methods.

CVD has become a majority method for graphene synthesis since 2009 [18]. This method is carried out by the use of highly volatile carbon sources under a high-vacuum and hightemperature $\left(\sim 1000{ }^{\circ} \mathrm{C}\right)$ environment with the use of an inert carrier gas. Common carbon precursors for the CVD graphene synthesis include gaseous (methane, acetylene, ethylene [19]), liquid (ethanol [20, 21], methanol [18, 22-24]), and solid (bio-carbon [25, 26], polymer [27], waste plastic [28]). Since graphene is obtained from secondary substrates [18-28], CVD is classified as 'bottom-up' synthesis [14-17]. Generally, the electrical conductivity of graphene synthesized by CVD is higher than that synthesized by the chemical method [22], and no reduction process is needed. The product is therefore very suitable for electronic 
devices. However, the approach also suffers from the shortcomings of low production yield and high cost $[14,29]$, which make this method unsuitable for large-scale graphene synthesis.

In contrast, the chemical method is relatively simple with a higher yield, which makes it popular for raw graphene production $[14,16]$. The Hummer's method is the most commonly used pathway, and the raw graphene obtained by this method is graphene oxide (GO). It was developed in the middle of the 20th century, when the graphitic oxide was the major product [30]. This method involved direct oxidation of graphite powder by a strong oxidation agent such as potassium permanganate $\left(\mathrm{KMnO}_{4}\right)$ to obtain graphite oxide, which can be said to be the origin of GO synthesis. However, the method also suffers from the weakness of poor product quality due to the formation of incomplete oxidized GO/graphite core/shell particles as final products throughout the graphite oxidation [31]. As a result, modifications of Hummer's reaction were proposed by different groups for quality improvement. In this method, the graphite was preoxidized into preoxidized graphite oxide by a mild oxidizing agent such as sodium nitrate $\left(\mathrm{NaNO}_{3}\right)$ or a strong oxidizing agent like a mixture of phosphorus pentaoxide $\left(\mathrm{P}_{2} \mathrm{O}_{5}\right)$ and potassium persulphate $\left(\mathrm{K}_{2} \mathrm{~S}_{2} \mathrm{O}_{8}\right)$ in sulphuric acid as the first step [32-35], which was the so-called Hummers and Offmann's method [36, 37]. After neutralizing and drying, the cleaned preoxidized product was then further oxidized into GO by a second oxidation with the use of $\mathrm{KMnO}_{4}$, followed by quenching with hydrogen peroxide $\left(\mathrm{H}_{2} \mathrm{O}_{2}\right)$ at the end of the oxidation [31-40]. The quenched products were then cleaned by hydrochloric acid and water in sequence, followed by dialysis cleaning in water. However, the use of $\mathrm{NaNO}_{3}$ may have the problem of toxic $\mathrm{NO}_{\mathrm{x}}$ evolution in the Hummer's reaction. Some research groups like Marcano et al. [41] further improved the process by using a larger amount of $\mathrm{KMnO}_{4}$ in $\mathrm{H}_{2} \mathrm{SO}_{4} / \mathrm{H}_{3} \mathrm{PO}_{4}$ solution without using $\mathrm{NaNO}_{3}$. This results in an increase in the total amount of hydrophilic oxidized GO when compared with those synthesized from traditional Hummer's method [41]. The purified raw GO was obtained by 
(1) simple filtration by water [41]; (2) freeze-drying the cleaned aqueous GO or direct storage without further treatment [42-46]; (3) oven-drying under vacuum or in an ambient environment $[47,48]$; or (4) drying in the air [32, 49]. The purified GO and exfoliated GO were very stable up to months and years [31]. However, such method exhibits a weakness of the metastability of the raw GO, especially under a low-temperature range of room temperature up to $50{ }^{\circ} \mathrm{C}[50-52]$. It is caused by the defects presented in the GO due to the $\mathrm{CO}_{2}$ elimination $[51,53]$. As a result, some research groups modified the synthesis conditions to obtain a better quality $\mathrm{GO}$, for example, by reducing the reaction temperature to $10{ }^{\circ} \mathrm{C}$ in the $\mathrm{KMnO}_{4}$ oxidation step, reducing the dosage of $\mathrm{KMnO}_{4}$, and increasing the reaction time from $3 \mathrm{hr}$ to $16 \mathrm{hr}$ [50]. Since GO was formed from the insertion of oxidized functional groups throughout the graphite oxide preparation, and the final GO nanosheets were obtained by separating the oxidized graphite oxide sheets by further oxidative exfoliation, the method was classified as 'top-down' in graphene synthesis [14-17].

The liquid-based shear-driven method is another common 'top-down' approach for graphene synthesis, which is also called the liquid phase exfoliation (LPE) method [15-17, 54, 55]. Graphite was converted into graphene by shearing of graphite in different kinds of solvent, including (1) organic solvents like N-methylpyrrolidone (NMP), N,N'dimethylformamide (DMF), dimethyl sulfoxide (DMSO) and so on [16]; (2) water [55]; and (3) ionic solvents like fulvic acid [54] as the first step, followed by mechanical post-treatment like ultrasonic, high-speed shear mixer, microwave, and so on $[16,54,55]$. Graphene was produced by the 'shearing' of graphite layers with the help of solvents to weaken the van der Waal's force between graphite layers. Finally, a mixture of mono-layer and few-layer pristine graphene was obtained when NMP was used as the solvent [16]. A similar result can also be achieved by the use of ionic liquid as demonstrated by Yang's team (1-5 layers graphene flakes with low defects) [54]. Liquid phase exfoliation from graphite was hard to achieve 
when using water as a solvent, which required the use of a stabilizer to avoid re-aggregation of the exfoliated graphene flakes [54]. Further studies were then carried out to solve this problem. One of the most recent demonstrations was by Tian et al. [55] who successfully synthesized graphene through water graphite exfoliation by replacing graphite powder with graphite oxide powder. The quality of graphene obtained can be improved and controlled by adjusting the ultrasonic time, speed of the homogenous disperser, and centrifugation rate [16, 55]. Since the amount of chemicals required for this process is relatively low and exfoliation can be achieved by the mechanical method as post-treatment, such a method was then regarded as a simple and low-cost method for large-scale low-defect pure graphene when compared with the chemical redox-pathway $[16,54]$. However, this method is a double-sided sword as it is also instrument- and solvent-sensitive, which may cause a lowering of the quality of the graphene produced, such as the defect formation in graphene flake $[16,55]$.

\section{Graphene-based products}

In the past decades, various kinds of graphene-based products for green energy research were developed by different techniques. These methods included chemical reactions, physical methods, and CVD. Different methods of preparing graphene-based products have been used to fit the specific needs and applications in green energy research. A detailed description of the nature of the reactions for graphene modification will be discussed in detail.

\subsection{Graphene derivatives from a wet chemical reaction}

Among different kinds of graphene-based product preparation from raw GO mentioned above, chemical synthesis was the mainstream of the methodology. It makes use of the special chemical properties of GO by the presence of oxidized carbon functional groups, which has been mentioned previously. So far, most of the chemically modified graphenes are 
2D structured, while 3D structured ones are relatively few in numbers due to its brand new nature. The difference between these two groups of graphene is that $2 \mathrm{D}$ graphene nanosheetbased products are composed of 2D nanosheets, while the 3D structured graphene products are composed of bulk blocks via stacking of the $2 \mathrm{D}$ graphene nanosheets throughout the $3 \mathrm{D}$ graphene network formation. These two categories of graphene can be further classified into 2 groups based on the nature of the chemical modification. They are pure reduced graphene and foreign materials modified graphene. Details are discussed below.

\subsubsection{Pure reduced graphene derivatives}

Pure graphene is obtained by the chemical reduction of GO obtained from the 'top-down' reactions like Hummer's method mentioned above, where the oxidized carbon functional groups (e.g. $\mathrm{C}=\mathrm{O}, \mathrm{O}-\mathrm{C}=\mathrm{O}$ and $\mathrm{C}-\mathrm{O}$ bonds) in pure $\mathrm{GO}$ were reduced by the reducing agent. This phenomenon was proved by the reduction in the intensity of HR-XPS peaks of the oxidized carbon groups (e.g. $\mathrm{C}=\mathrm{O}, \mathrm{O}-\mathrm{C}=\mathrm{O}$ and $\mathrm{C}-\mathrm{O}$ bonds) from XPS characterization of these products against that from the corresponding pure GO samples [56-60]. The process is the same for $2 \mathrm{D}$ or $3 \mathrm{D}$ graphenes. The chemically reduced product is called reduced graphene oxide (rGO), while its 3D bulk block form is called graphene hydrogel (GH). The origin of such difference was the process because $2 \mathrm{D}$ rGO was obtained from the chemical reduction of graphene under stirring [56,61], while 3D GH was obtained from stationary reduction, mainly the hydrothermal or chemical reduction [57-63]. In the early years, strong reducing agents like hydrazine $\left(\mathrm{N}_{2} \mathrm{H}_{4}\right)$, boron hydride $\left(\mathrm{BH}_{4}{ }^{-}\right)$species, lithium aluminum hydride $\left(\mathrm{LiAlH}_{4}\right)$, amine group compounds, iodine-containing compounds, hypophosphorous acid, or formaldehyde were common reducing agents to achieve the goal ([41, 56, 61] (2D) and [5764] (3D)). Its major advantage is that the reaction time needed is short, but it has the adverse effect of strong toxicity arising from the reducing agent itself. In addition, due to the strong 
reaction between $\mathrm{GO}$ nanosheets and some of the reducing agents mentioned above, the formation of $\mathrm{CO}_{2}$ gas bubble during the reduction of $\mathrm{GO}$ into reduced graphene would take place throughout the GH formation, which results in the breaking of GH under hydrothermal reduction [59]. As a result, the use of a green or a non-toxic reducing agent is the alternative, which includes L-ascorbic acid or the so-called vitamin C (VC), ethanol, and ethylene diamine $[32,38,42,59,65,66]$. The product obtained by this group of reducing agents has the advantages of having no toxic residue after reduction $[32,38,42,59,65,66]$, and the shortcoming of GH breaking throughout the reaction can be avoided [42, 59]. Final bulk graphene aerogel (GA) was obtained by freeze-drying GH synthesized from methods previously mentioned $[42,57-60,62,63,65,66]$. Some of the cases did not even require the use of a reducing agent for the GA synthesis $[67,68]$. The shape of the GA can also be customized by the shape of the reactor or the use of a regular pillar pattern throughout the hydrothermal reaction as demonstrated by Worsley's group [64] and Wang's group [66] from illustrations in Figures 1 and 2, respectively. Such a technique can also be used in the GA/Ni foam (NF) synthesis [32, 33, 67-71], as reflected in the digital images and microscopic image of GOA/NF and GA/NF shown in Figures 3 and 4 [33]. The demonstrations mentioned above provided the possibility of designing a substrate-free electrode with shape and pattern customized for the battery research at the device level. 


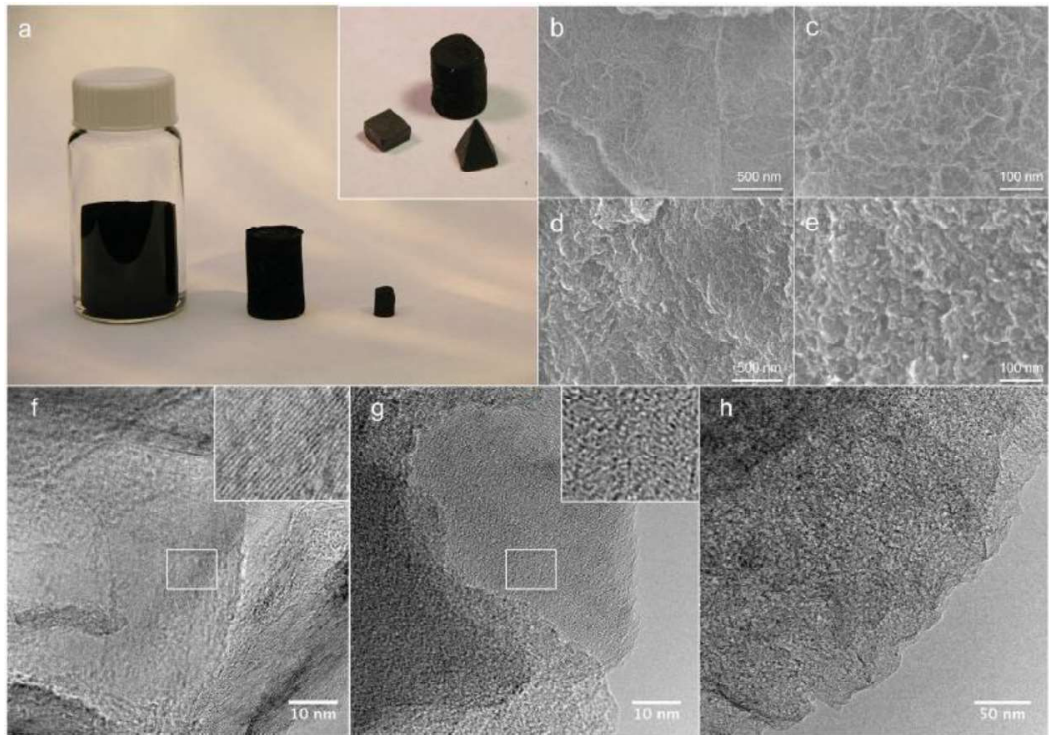

Fig 1. a) Photograph of a reduced GU wet gel betore drying (lett), after supercritical drying (middle), and after ambient drying (right). For reference, the wet gel is in a $20 \mathrm{ml}$ vial. Inset shows a GDC cylinder (cast), prism (machined), and pyramid (machined). SEM images of fracture surfaces of $b, c)$ the GMA and d,e) GDC at b,d) low and c,e) high magnification. f) TEM images of commercial graphite at high magnification. Inset is a zoom-in of white box area and is $10 \mathrm{~nm}$ in width. g) TEM image of GDC at high magnification. Inset is a zoom-in of white box area and is $10 \mathrm{~nm}$ in width. h) Low magnification TEM image of GDC.[64] (Wiley Interscience)
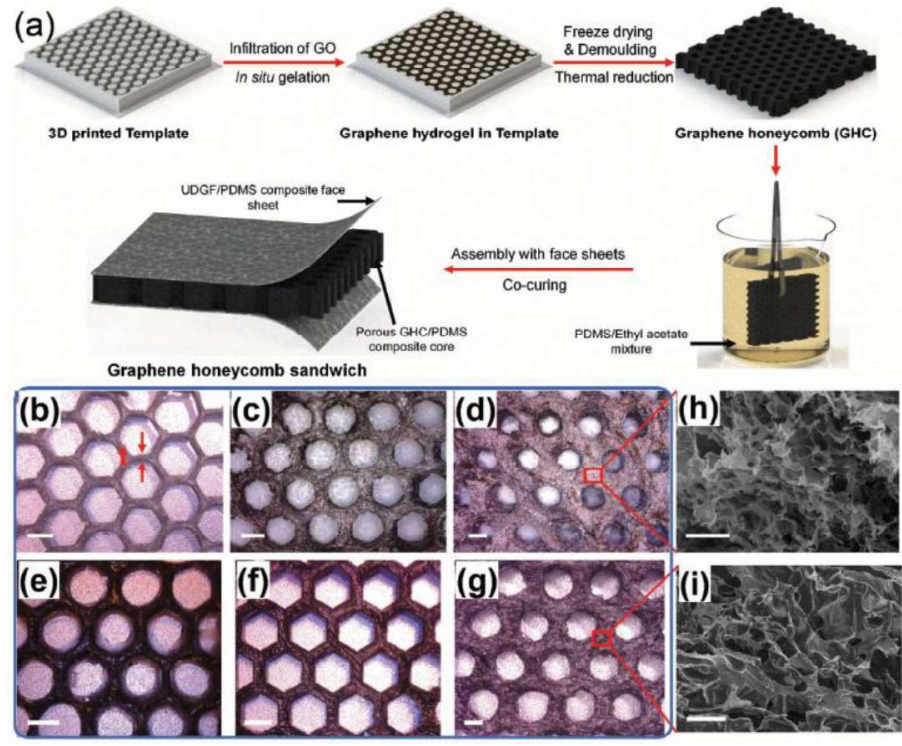
Fig 2. a) Fabrication of GHCs and GHC sandwiches; Optical images of b-d) GHCs with different GA wall thicknesses, $t$, ranging from 0.25 to $1 \mathrm{~mm}$, and $\mathrm{e}-\mathrm{g}$ ) the corresponding GHC/PDMS composites; SEM images of h) GHC and i) GHC/PDMS composites.[66] (Wiley Interscience)

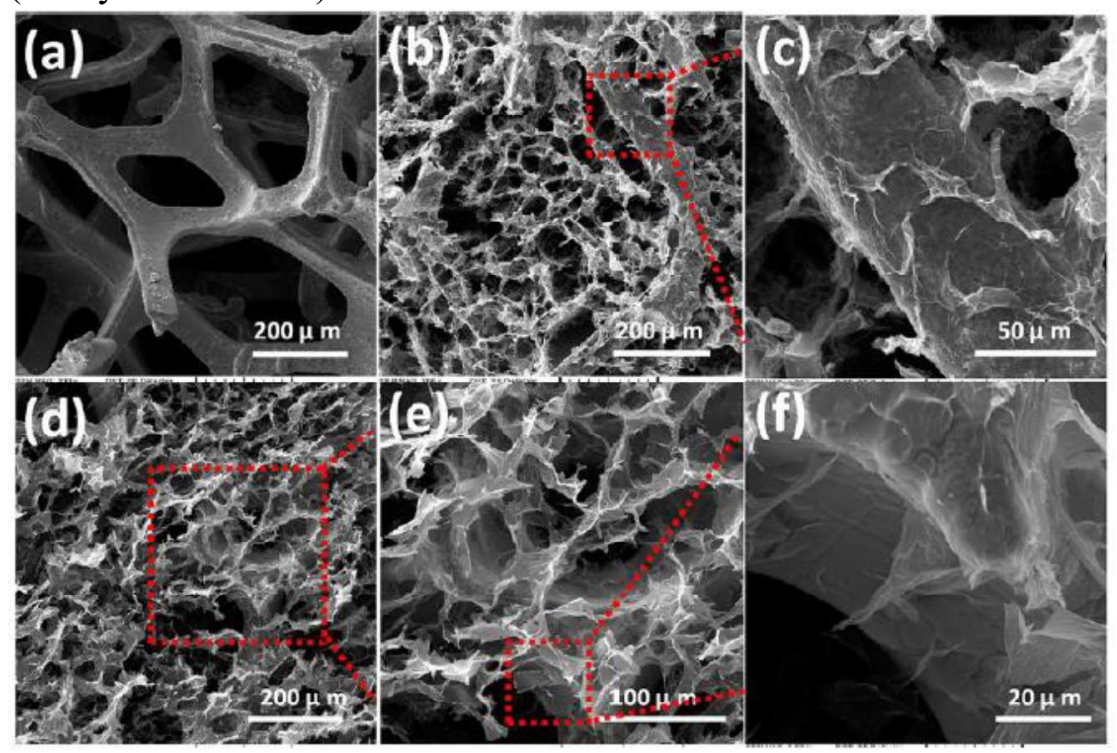

Fig 3. SEM images of (a) NF, (b, c) as-prepared GOA@NF at diff erent magnifications, and (d-f) as-prepared GA@NF at diff erent magnifications.[33] (ACS)

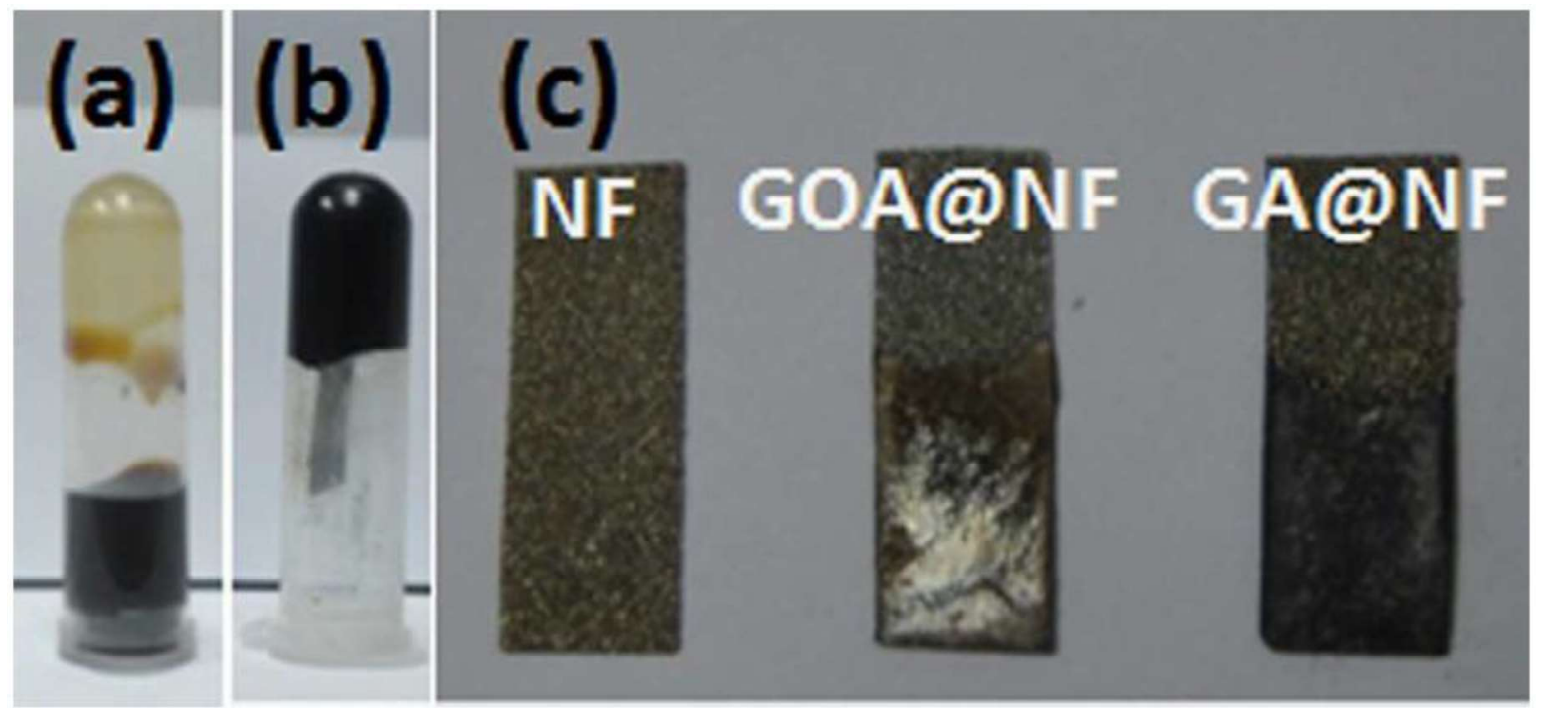

Fig 4. Photographs of (a) GO aqueous dispersions, (b) GO hydrogel with the insert of a NF sheet, and (c) NF, GOA@NF, and GA@NF.[33] (ACS) 


\subsubsection{Foreign materials modified graphene}

Foreign materials (i.e. metal/metal oxide base, or non-metallic elements doped)-loaded graphene was obtained by direct chemical reduction of foreign materials. Generally, metal modified graphene (M/rGO) $[38,43-46,48,60,61,72-126]$ and metal oxide modified graphene $\left(\mathrm{MO}_{\mathrm{x}} / \mathrm{rGO}\right)[7,127-137]$ were synthesized from the reaction between metal ions and GO. Similarly, $\mathrm{MO}_{\mathrm{x}} / \mathrm{rGO}$ can also synthesized by reacting $\mathrm{MO}_{\mathrm{x}}$ with $\mathrm{GO}[22,23,138$ 143]. Non-metal elements-doped graphene was obtained from a reaction between GO and non-metal $(B, N$, or S) dopants $[7,56,73,144-157]$. Such a principle applies to both $2 \mathrm{D}$ and 3D graphene substrate structures. The only difference is that foreign materials-loaded 2D graphenes are obtained by the continuous mixing of GO with foreign materials via a stirring environment with different treatment techniques. Among them, stirring under heating was the most commonly used technique for the $\mathrm{M} / \mathrm{rGO}[43-46,48,61,72,76,77,84,86,87,90,93$, $97,100,102,115-117,123,126], \mathrm{MO}_{\mathrm{x}} / \mathrm{rGO}[7,127-135,140-142]$, and non-metal elementdoped rGO [56, 145-154], while some of the continuous stirring synthesis under heating was (1) a reflux reaction $[45,109,110,119]$, (2) use of the gaseous reducing agent of CO [36, 158], or (3) ion displacement reaction $[114,115]$ for $\mathrm{M} / \mathrm{rGO}$ preparation. Some of the $2 \mathrm{D}$ structured graphene products via thermal reaction synthesis do not require the use of stirring; these reactions include (1) hydrothermal reaction [89, 98, 124, 155], (2) microwave-assisted synthesis [85, 88, 137], and (3) heat flux with inert gas [159]. In contrast, 3D graphene-based products like GA-based products were obtained under a stationary environment for a period in terms of hours once a reducing agent was added to the $\mathrm{GO} /$ loading materials mixture [38, $60,73,78,111,113,121,122,144,156,157,160,161]$. The dry products from chemical synthesis were obtained by normal drying under heat $[7,56,61,75-77]$, or freeze-drying [73, $78,121,122,138,142,144,156,157,160,161]$. Similar to the reduced graphene, chemically synthesized foreign materials-loaded graphene are obtained from a reducing agent composed 
of $\mathrm{N}_{2} \mathrm{H}_{4}$, amine-based compounds, or $\mathrm{BH}_{4}^{-}$via simultaneous reduction under hydrothermal reaction environment as listed in Table $1[44,48,56,60,61,76,79,80,84,86,91,92,96,97$, $99,100,102,103,107,109,111,113,124,125,157,162]$. However, a problem similar to the pure reduced graphene preparation exists, such as the use of a toxic reducing agent in nature under harsh reaction conditions, which violate the concept of user-friendliness in the graphene products synthesis. Green elements, such as VC, ethanol, ethyl glycol (EG), 1,2propanediol (PD), a mixture of ethyl glycol/isopropyl alcohol (EG/IPA), and formic acid, were then imported to foreign materials-loaded graphene. These were reducing agents commonly used for $\mathrm{M} / \mathrm{rGO}$ or $\mathrm{M} / \mathrm{GA}$ products as listed in Table $1[38,43,45,46,72,73,75$, $78,81-83,85,87-90,93,94,101,104-106,108,110,163,164]$, or dopant itself for the foreign elements-doped graphene products [7, 144-147]. The quality of these graphene-based products was comparable to those obtained from traditional reducing agents $[43,45,73]$. This is revealed from an example shown in Figure 5, where the bulk platinum nanocrystal-loaded $\mathrm{N}$-doped graphene aerogel (PtNCs@NGA) after the simultaneous reduction of GO, $\mathrm{PtCl}_{6}^{2-}$ and dopant (dopamine) by EG under hydrothermal condition was a black cylindrical block [73]. At the same time, the successful loading of high crystalline metal nanoparticles (PtNCs) was proved by the presence of black spots on the surface of the graphene nanosheet from the HR-TEM image of PtNC/NGA [73]. In contrast, some of the metal-loaded GA products are reducing agent-free reaction $[121,122]$, such as Pd-loaded graphene aerogel (GA/Pd NP) synthesized by Yun et al. [122], which have an appearance similar to those synthesized by the traditional pathway (i.e. reducing agent-assisted reaction) described above [122]. This is illustrated in the SEM and TEM images shown in Figure 6, where Pd NPs are highly distributed on the surface of graphene nanosheets in the GA/Pd NP array [122].

Table 1. List of the $\mathrm{M} /$ graphene-based products produced by chemical reduction with corresponding reducing agent and surface properties.

\begin{tabular}{lllll}
\hline (Catalyst)/Graphene host & Reducing & agent & Electrochemical active & Ref \\
\hline
\end{tabular}




\begin{tabular}{|c|c|c|c|}
\hline & (Green/Toxic) & surface area $\left(\mathrm{m}^{2} \mathrm{~g}^{-1}\right)$ & \\
\hline $\mathrm{Pd} / \mathrm{rGO} ; \quad \mathrm{PdRu} / \mathrm{rGO}$ & $\mathrm{BH}_{4}^{-}$(Toxic) & $58.1 ; 52.0 ; 64.9 ; 61.6$ & [44] \\
\hline $\mathrm{PdSn} / \mathrm{rGO} ; \mathrm{PdIr} / \mathrm{rGO}$ & & & \\
\hline $\begin{array}{l}\mathrm{Pd} \quad(10 \quad \mathrm{~nm}) / \mathrm{rGO} ; \quad \mathrm{Pd} \quad(3 \\
\mathrm{nm}) / \mathrm{rGO}\end{array}$ & $\mathrm{BH}_{4}^{-}$(Toxic) & $182.56 ; 162.56$ & {$[48]$} \\
\hline $\mathrm{Pd}_{1} \mathrm{Cu}_{1} / \mathrm{rGO}$ & $\mathrm{BH}_{4}^{-}$(Toxic) & 86 & {$[76]$} \\
\hline $\mathrm{Pt} / \mathrm{rGO}$ & $\mathrm{BH}_{4}^{-}$(Toxic) & 141.6 & [86] \\
\hline $\mathrm{Pt} / \mathrm{rGO}$ & $\mathrm{BH}_{4}^{-}$(Toxic) & 44.6 & [91] \\
\hline $\mathrm{Pd}_{1} \mathrm{Ni}_{1}-\mathrm{NNs} / \mathrm{rGO} ; \mathrm{Pd} / \mathrm{rGO}$ & $\mathrm{BH}_{4}^{-}$(Toxic) & $98.2 ; 67.2$ & [96] \\
\hline $\mathrm{Pd}-\mathrm{Au}(1: 1) / \mathrm{rGO} ; \mathrm{Pd} / \mathrm{rGO}$ & $\mathrm{BH}_{4}^{-}$(Toxic) & N/A & [99] \\
\hline Pd-Ag (1:1)/rGO-SB & $\mathrm{BH}_{4}^{-}$(Toxic) & $\mathrm{N} / \mathrm{A}$ & {$[100]$} \\
\hline rGO-Pd; rGO-Pt & $\mathrm{BH}_{4}^{-}$(Toxic) & $\mathrm{N} / \mathrm{A}$ & {$[102]$} \\
\hline $\mathrm{Pt} / \mathrm{rGO}$ & $\mathrm{BH}_{4}^{-}$(Toxic) & 98 & {$[109]$} \\
\hline $\mathrm{Pd} / 3 \mathrm{DGA}$ & $\mathrm{BH}_{4}^{-}$(Toxic) & $\mathrm{N} / \mathrm{A}$ & {$[113]$} \\
\hline Ni/2D-rGO; Ni/3D-rGO & $\mathrm{N}_{2} \mathrm{H}_{4}$ (Toxic) & 463 (BET); 883 (BET) & {$[61]$} \\
\hline $\mathrm{Pt} @ \mathrm{Pd} / \mathrm{rGO} ; \mathrm{Pt} / \mathrm{rGO} ; \mathrm{Pd} / \mathrm{rGO}$ & $\mathrm{N}_{2} \mathrm{H}_{4}$ (Toxic) & $15.11 ; 10.42 ; 10.59$ & [79] \\
\hline Pd-Pt ANFs/rGO & $\mathrm{N}_{2} \mathrm{H}_{4}$ (Toxic) & 200.32 & [80] \\
\hline $\mathrm{Ni} / \mathrm{rGO}$ & $\mathrm{N}_{2} \mathrm{H}_{4}$ (Toxic) & $\mathrm{N} / \mathrm{A}$ & [84] \\
\hline $\mathrm{Pd} / \mathrm{Cu} / \mathrm{rGO}$ & $\mathrm{N}_{2} \mathrm{H}_{4}$ (Toxic) & 20.22 & [92] \\
\hline $\mathrm{rGO} / \mathrm{Pd}$ & $\mathrm{N}_{2} \mathrm{H}_{4}$ (Toxic) & $\mathrm{N} / \mathrm{A}$ & [103] \\
\hline PtPd NFs-rGO & $\mathrm{N}_{2} \mathrm{H}_{4}$ (Toxic) & 26.33 & {$[107]$} \\
\hline $\mathrm{Ni} / \mathrm{GA}$ & $\mathrm{N}_{2} \mathrm{H}_{4}$ (Toxic) & $\mathrm{N} / \mathrm{A}$ & {$[111]$} \\
\hline 3DGFs & $\mathrm{N}_{2} \mathrm{H}_{4}$ (Toxic) & $\mathrm{N} / \mathrm{A}$ & {$[162]$} \\
\hline $\mathrm{Pd}_{70} \mathrm{Ag}_{30} @ \mathrm{rGO}$ & $\mathrm{N}_{2} \mathrm{H}_{4}$ (Toxic) & 13.58 & {$[125]$} \\
\hline $\mathrm{Pd} / \mathrm{rGO}$ & Oleylamine (Toxic) & N/A & [97] \\
\hline $\mathrm{Au} / \mathrm{GH}$ & $\begin{array}{l}\text { Triethylenetetraamine } \\
\text { (Toxic) }\end{array}$ & $\mathrm{N} / \mathrm{A}$ & [112] \\
\hline $\mathrm{Pt}_{75.4} \mathrm{Cu}_{24.6}$ Alloy/rGO & $\begin{array}{l}\text { Hydroxylamine } \\
\text { hydrochloride (Toxic) }\end{array}$ & $\mathrm{N} / \mathrm{A}$ & {$[124]$} \\
\hline $\mathrm{Pt}_{68.2} \mathrm{Cu}_{31.8}$ Alloy/rGO & $\begin{array}{l}\text { Hydroxylamine } \\
\text { hydrochloride (Toxic) }\end{array}$ & $\mathrm{N} / \mathrm{A}$ & {$[124]$} \\
\hline $\mathrm{Pt} / \mathrm{GH}$ hybrid & L-ascorbic acid (Green) & N/A & {$[38]$} \\
\hline $\mathrm{Pd} @ \mathrm{Pt} / \mathrm{rGO} ; \mathrm{PdPt} / \mathrm{rGO}$ & L-ascorbic acid (Green) & $29.94 ; 37.55$ & [43] \\
\hline $\begin{array}{l}\mathrm{Pd} / \mathrm{rGO}, \quad \mathrm{Pt} / \mathrm{rGO}, \quad \mathrm{Au} / \mathrm{rGO}, \\
\mathrm{Ag} / \mathrm{rGO}\end{array}$ & L-ascorbic acid (Green) & $\mathrm{N} / \mathrm{A}$ & [45] \\
\hline $\mathrm{Au} @ \mathrm{Pd}-\mathrm{G}, \mathrm{Au}-\mathrm{G}, \mathrm{Pd}-\mathrm{G}$ & L-ascorbic acid (Green) & $\mathrm{N} / \mathrm{A}$ & [81] \\
\hline rGOs-PtPd NHs & L-ascorbic acid (Green) & 18.5 & [82] \\
\hline $\begin{array}{l}\text { Pt1Pd3NPs/rGO; PtNPs/rGO; } \\
\text { PdNPs/rGO }\end{array}$ & L-ascorbic acid (Green) & $23.34 ; 21.93 ; 13.87$ & [83] \\
\hline $\mathrm{PdNCs} / \mathrm{rGO}$; PdNPs/rGO & L-ascorbic acid (Green) & $50.28 ; 25.64$ & [94] \\
\hline $\mathrm{PtPd} / \mathrm{rGO} ; \mathrm{Pt} / \mathrm{rGO} ; \mathrm{Pd} / \mathrm{rGO}$ & L-ascorbic acid (Green) & $83.1 ; 16.3 ; 12.5$ & [104] \\
\hline $\mathrm{Pd} / \mathrm{GA} / \mathrm{NF}$ & L-ascorbic acid (Green) & $\mathrm{N} / \mathrm{A}$ & [164] \\
\hline $\mathrm{Pd}_{1} \mathrm{Pt}_{1.03} / \mathrm{GA} / \mathrm{NF}$ & L-ascorbic acid (Green) & $\mathrm{N} / \mathrm{A}$ & {$[165]$} \\
\hline $\mathrm{Nd}_{2} \mathrm{O}_{5} / \mathrm{GF} ; \mathrm{Nd}_{2} \mathrm{O}_{5} / \mathrm{HGF}$ & $\begin{array}{l}\text { Sodium ascorbate } \\
\text { (Green) }\end{array}$ & 63 (BET); 83 (BET) & [8] \\
\hline PtNFs-rGO & Ethanol (Green) & 72.1 & {$[46]$} \\
\hline $\mathrm{Pd} / \mathrm{N}-3 \mathrm{D}-\mathrm{rGO}$ & Ethanol (Green) & $390(\mathrm{BET})$ & {$[75]$} \\
\hline $\begin{array}{l}\text { PtPdNPs/rGO; PdNPs/rGO; } \\
\text { PtNFs/rGO }\end{array}$ & Ethanol (Green) & $\mathrm{N} / \mathrm{A}$ & [93] \\
\hline PtNCs@NGA & EG (Green) & 1750 (BET), 871 & {$[73]$} \\
\hline
\end{tabular}




\begin{tabular}{|c|c|c|c|}
\hline $\mathrm{Pt} / \mathrm{rGO}$ & EG (Green) & 36.27 & [87] \\
\hline $\mathrm{Pt} / \mathrm{ND} / \mathrm{rGO}-1500$ & EG (Green) & $137.9 ; 125.1$ & [88] \\
\hline $\mathrm{Pt} / \mathrm{ND} / \mathrm{rGO}-1200$ & & & \\
\hline $\mathrm{rGONP} / \mathrm{Pt} ; \mathrm{rGO} / \mathrm{Pt}$ & EG (Green) & $63.0 ; 53.6$ & [89] \\
\hline $\begin{array}{l}\mathrm{Pd}-\mathrm{Ag} / \mathrm{rGO} \\
\mathrm{Ag} / \mathrm{rGO}\end{array}$ & EG (Green) & $\mathrm{N} / \mathrm{A}$ & [90] \\
\hline $\mathrm{Pd}-\mathrm{Cu} / \mathrm{rGO} ; \mathrm{Pd} / \mathrm{rGO} ; \mathrm{Cu} / \mathrm{rGO}$ & EG (Green) & $\mathrm{N} / \mathrm{A}$ & [101] \\
\hline $\mathrm{Pd}-\mathrm{CuNC} / \mathrm{rGO}$ & EG (Green) & 49.2 & [105] \\
\hline rGO-Pd; rGO-Pt & EG (Green) & $\begin{array}{l}544.3 \quad(\mathrm{BET}) ; 478.3 \\
(\mathrm{BET})\end{array}$ & [108] \\
\hline $\mathrm{Pt} / \mathrm{rGONP}$ & EG (Green) & $\sim 40$ & [110] \\
\hline $\begin{array}{l}40 \% \mathrm{Pd}-10 \% \\
\mathrm{Pd} / \mathrm{rGO}\end{array}$ & EG (Green) & N/A & [163] \\
\hline $\mathrm{Pt} / \mathrm{rGO}-5 ; \mathrm{Pt} / \mathrm{rGO}-10$ & PD (Green) & $85.71 ; 65.61$ & {$[85]$} \\
\hline $\mathrm{Pt} / \mathrm{HGF}$ & EG/IPA (Green) & 340.65 (BET), 92.58 & {$[78]$} \\
\hline rGO-Pt; rGO-PtPd & Formic acid (Green) & $38.6 ; 23.6$ & {$[72]$} \\
\hline $\begin{array}{l}\mathrm{PdPt} @ \mathrm{Pt} / \mathrm{rGO} ; \quad \mathrm{PtPd} / \mathrm{rGO} ; \\
\mathrm{Pt} / \mathrm{rGO} ; \mathrm{Pd} / \mathrm{rGO}\end{array}$ & Formic acid (Green) & $58.9 ; 20.5 ; 17 ; 14$ & [106] \\
\hline
\end{tabular}

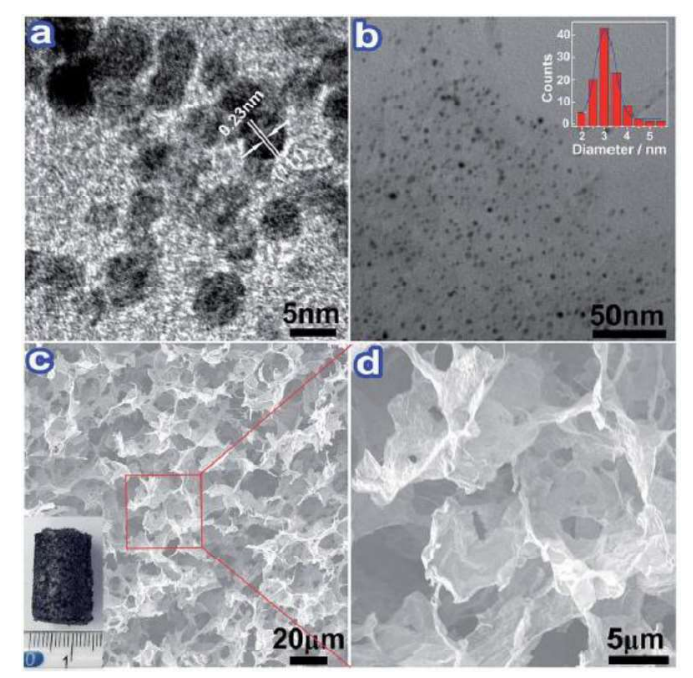

Fig 5. (a) HRTEM and (b) TEM images (inset, size-distribution histogram) of PtNCs in NGA; (c) SEM image (inset, photograph of bulk PtNCs@NGA) and (d) magnified SEM image of PtNCs@NGA.[73] (RSC) 

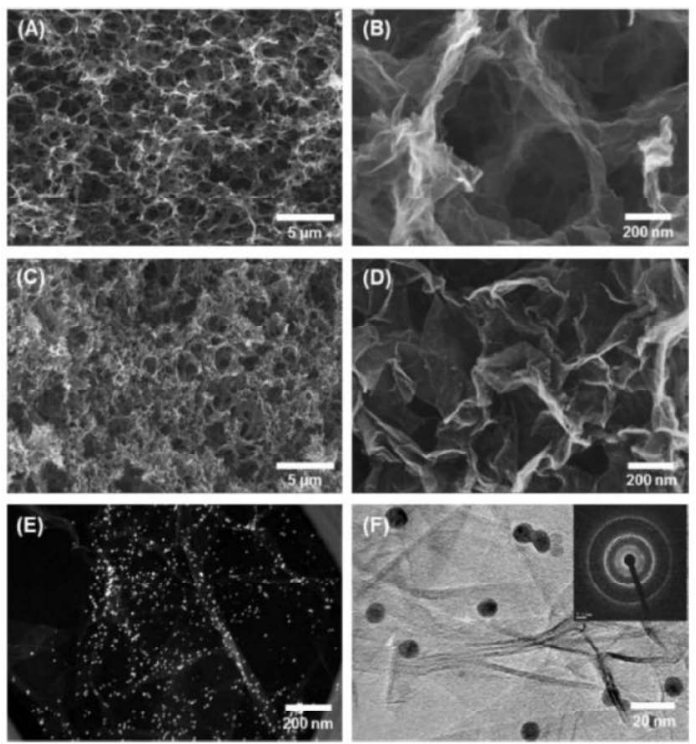

Fig 6. Low and high magnificent SEM images of (a) and (b) of GAs, and (c) and (d) of GA/Pd NP hybrids. (e) Dark-field TEM and HR-TEM images of GA/Pd NP hybrids. Inset of SAED pattern of GA/Pd NP hybrids.[122] (Elsevier B. V.)

\subsection{Graphene derivatives from CVD method}

Relative to the mainstream of the chemical method for the synthesis of the graphene derivatives, the CVD method is an alternative that was used for the synthesis of $2 \mathrm{D}$ and $3 \mathrm{D}$ graphene derivatives, such as pure graphene foam (GF) $[166,167]$, metal modified [20], $\mathrm{MO}_{\mathrm{x}}$ modified graphene [24, 168], and foreign elements-doped graphene [21, 169, 170]. In general practice, the substrate was required for the synthesis of foreign materials-loaded graphene via CVD, which was similar to the procedure in CVD-synthesized pure graphene. Examples include $\mathrm{Cu}$ foil [21], NF [168, 170], silicon wafer [20], and glass plate [24]. The size of the template used was also small in size $\left(2 \mathrm{~cm}^{2}\right.$ to $\left.6.3 \mathrm{~cm}^{2}\right)[20,21,24]$. There was an exception that boron nitrile-doped graphene quantum dots (BN-GQD)/G and $\mathrm{BN}-$ doped graphene (BNG) synthesized by CVD were carried out in a quartz boat throughout the synthesis, and the product was directly collected in powder form [169]. The dopant used can be solid (e.g. Zn, 
boric acid) [24, 169], or gaseous dopant (e.g. $\mathrm{NH}_{3}$ ) [21, 169]. By definition of graphene synthesis mentioned in the previous section $[14-16,19,171-178]$, the synthesis of these chemically modified graphenes can be regarded as an extension of 'bottom-up' graphene synthesis. Appearances of the CVD-synthesized foreign materials-loaded graphene products were similar to those synthesized by chemical synthesis, which is mentioned in the previous section. It was revealed from the STEM of Pt-loaded graphene nanoflake films (GNFs) illustrated in Figure 7 [20], where Pt nanoclusters were evenly distributed on the GNFs surface. Even though it was instrumental in the compact-sized electrode research, this technique has the shortcoming that only limited product size can be synthesized on the substrate. Also, such a method is costly and low yield for device fabrication [29, 179-181]. These limited its application in large-sized energy devices.
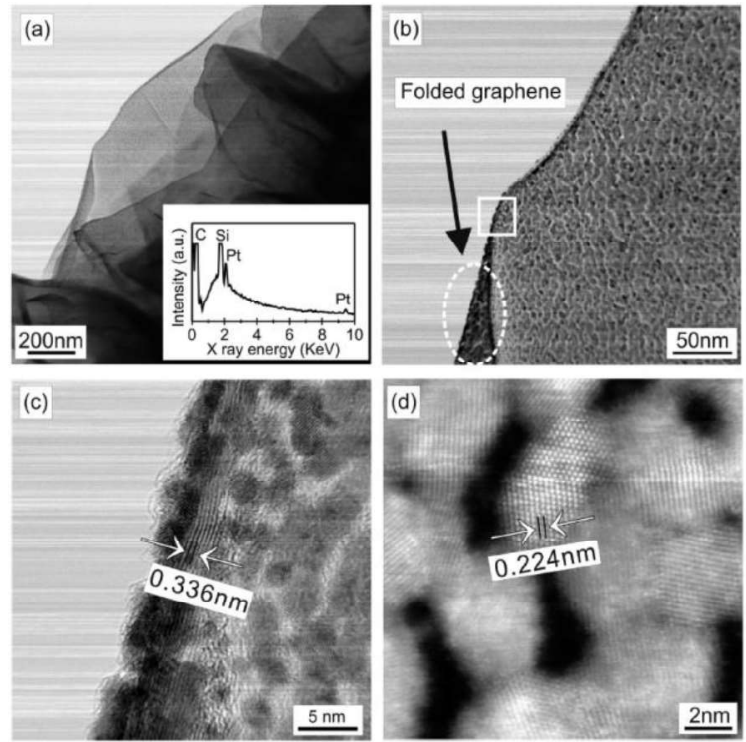

Fig 7. (a, b) Low-magnification STEM images of $2.1 \mathrm{~nm}$ thick Pt nanoclusters coated GNFs. Inset is the corresponding EDS spectrum taken in SEM. High-resolution STEM (c, enlarged image of the marked square area of (b)) and HAADF (d) images of $2.1 \mathrm{~nm}$ Pt nanoclusters on GNFs, revealing 2-4 nm monolayer Pt nanoclusters well intercoupled on both basal and edge planes of high-quality GNFs.[20] (ACS) 


\subsection{Graphene-based products by other methods}

Besides chemical synthesis and CVD methods, other non-chemical methods like physical methods have also been developed for the synthesis of graphene-based products, such as (1) electrodeposition [37, 47, 49, 128, 182-192], (2) ultrasonic synthesis [139, 143, 193-196], (3) direct mixing with simple heating [22, 23, 197-200], and (4) direct mixing in an ambient environment $[69,74,75,118,120,125]$. These methods were mainly used for the foreign material-loaded graphene-based materials, especially metal (2D graphene: [37, 47, 49, 74, 75, $118,120,182-188,193-195])$ or $\mathrm{MO}_{\mathrm{x}}(2 \mathrm{D}$ graphene: $[128,139,189,190,197-200]$, 3D graphene: $[22,23,69,196])$ modified graphene. In contrast, such a method was seldom used for preparing foreign element-doped graphene [191]. Another common feature is that the final graphene products were either in liquid media $[37,74,75,118,120,139,184,189,193-$ $197]$ or deposited on a secondary substrate for $2 \mathrm{D}$ graphene $[47,49,128,182,183,185-188$, 190, 191, 198-200] and 3D GA [69] and porous 3D GF products [22, 23, 192]. From the comparison of TEM images of the metal-loaded graphene synthesized by electrodeposition ( $\mathrm{Pd}$ on $\mathrm{rGO} / \mathrm{CFP}[47])$ and direct mixing (FS-Pd/GO [118]) as shown in Figures 8 and 9, there was almost no difference in the morphology of Pd-loaded graphene. This showed the feasibility of the foreign materials-loaded graphene synthesized by totally physical pathways. The fabrication of modified graphenes by this method was comparatively easy, which made it suitable for device fabrication. Even though such methods are relatively easier than CVD or the traditional chemical synthetic pathway, as mentioned in previous sections, process requirement was also complicated when using such materials for device fabrication. Such a drawback is especially obvious for the application of metal- or metal oxide-loaded GF as DSSC or fuel cell electrodes because removal of the NF skeleton is required [22, 23, 192]. 


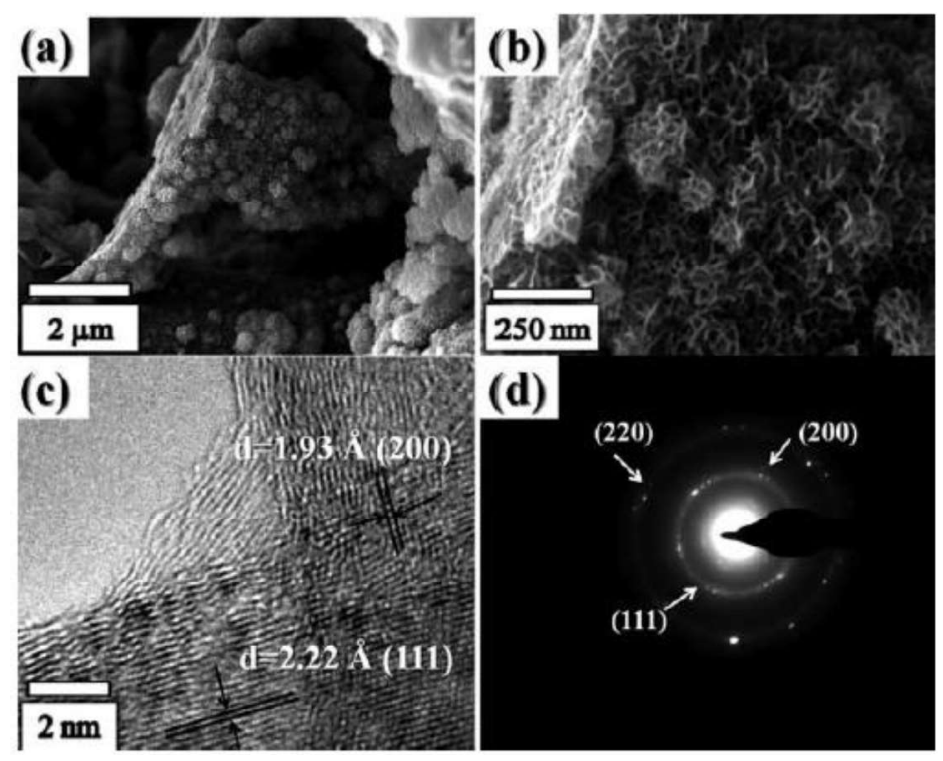

Fig 8. (a) Lower- and (b) higher-magnification SEM images of as-electrodeposited Pd on the rGO/CFP electrode, (c) HRTEM image, and (d) electron diffraction pattern of aselectrodeposited Pd.[47] (RSC)
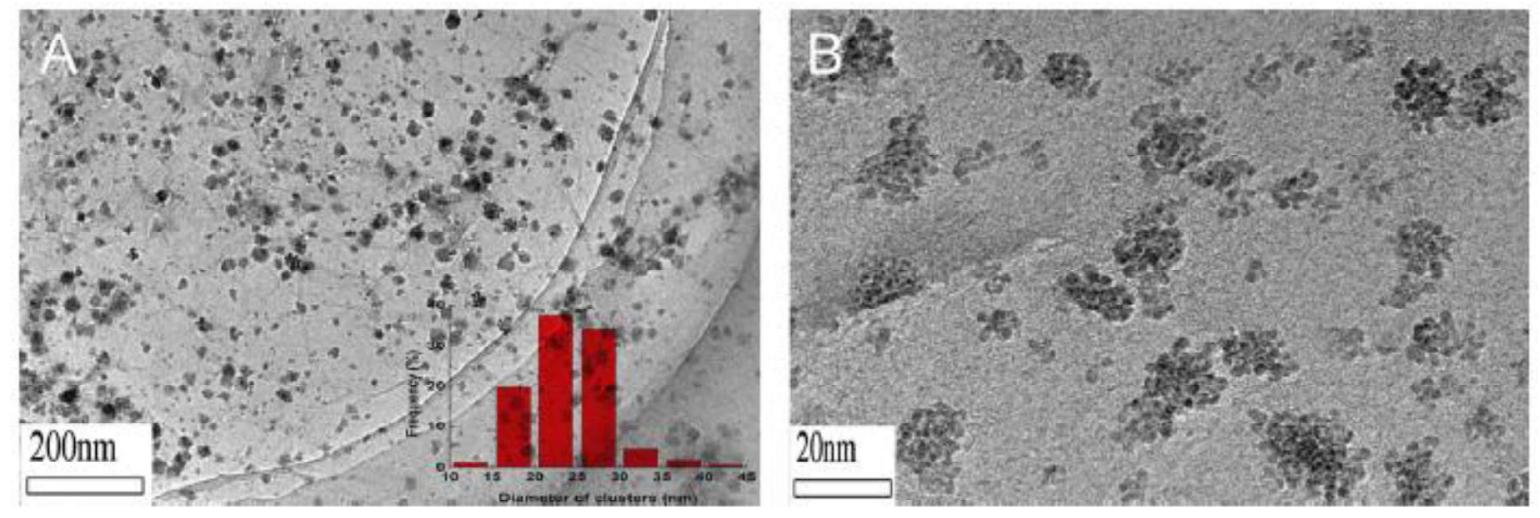

Fig 9. TEM images of FS-Pd/GO composites in different scales. The inset of (A) is the sizehistogram of FS-Pd.[118] (Elsevier B. V.)

\section{Graphene-based materials for green energy applications}

\subsection{Fuel cells and metal-air batteries}

The foreign materials-loaded graphene played an important role in green energy research. The main reason is that the interaction between graphene nanostructure and loaded materials 
is usually enhanced upon modification with graphene. Electrocatalytic fuel cell and battery research is a hot topic in green energy development. Fuel cells can reduce fossil fuel demand by generating electricity via electrochemical oxidation of renewable alcoholic fuels like methanol, ethanol, and glucose, as well as gaseous fuel hydrogen produced by water-splitting. In addition to the strong catalytic activity of fuel cell catalysts, such as monometallic metal and bimetallic precious metal catalysts, as well as easy modification properties of graphene with those materials, graphene-based materials have become a very popular candidate as an electrocatalyst. Graphene-based electrocatalysts, no matter whether they involve 2D or 3D graphene, are classified into two main groups. The first corresponds to anodic catalysts for alcoholic fuel (methanol, ethanol, and glucose) in alcoholic fuel cells or hydrogen oxidation in hydrogen fuel cells. The second corresponds to cathodic catalysts for oxygen reduction reactions (ORR) for alcoholic fuel cells, hydrogen fuel cells, or metal-air batteries.

\subsubsection{Direct alcohol fuel cells}

First, in the alcoholic fuel electrooxidation, methanol oxidation reaction (MOR) was the most popularly studied topic. In the past decade, metal-loaded 2D graphene (M/rGO) and metal-loaded graphene aerogel (M/GA) were the most popular electrocatalytic materials for the MOR. Platinum was the most commonly used metal candidate for the $2 \mathrm{D} \mathrm{M} / \mathrm{rGO}[43,46$, $79,80,82,83,85-89,91,102,104,117,158,159,187,188]$, or 3D graphene-based [78, 192] anodic electrocatalysts (Table 2). Results from different groups showed that the Pt-loaded graphene-based catalysts (Pt/rGO or Pt/GA based) exhibited stronger electrochemical activity in the $\mathrm{MeOH}$ half-cell scaled electrooxidation than the commercial $\mathrm{Pt} / \mathrm{C}, \mathrm{Pt} /$ carbon black or even pure $\mathrm{Pt}$, as reflected in the maximum anodic sweep current density $\left(\mathrm{J}_{\mathrm{f}}\right)$ of $2540 \mathrm{~A} \mathrm{~g}^{-1}$ (unit mass) [43] or $30 \mathrm{~mA} \mathrm{~cm}^{-2}$ (unit area) [87], with strong tolerance towards $\mathrm{CO}\left(\mathrm{I}_{\mathrm{f}} / \mathrm{I}_{\mathrm{b}}\right)$ as high as 6.6 [82]. However, due to the toxicity of methanol itself, ethanol (EtOH) has become 
an alternative fuel in the electrooxidation study. Same as the case of MOR, Pt-based graphene electrocatalyst was extensively used in the past years, showing strong activity in EtOH electrooxidation (EOR) $[49,93,104,106]$. The metal candidate was replaced by nonplatinum monometallic catalyst or bimetallic catalyst at a later stage for the elimination of carbon monoxide $(\mathrm{CO})$ poisoning from $\mathrm{MOR}$ and EOR in the $\mathrm{Pt} /$ graphene-based anodic catalyst due to the blockage of the active surface site of Pt by the $\mathrm{CO}$ molecules, which shortens the lifetime of the Pt/graphene catalysts. Non-platinum monometallic catalysts like $\operatorname{Pd}([37,44,48,49,74,79-83,90,93-104,106,114,115,118,119,158,163,184,194](2 \mathrm{D}$ $\mathrm{M} / \mathrm{rGO}),[113,164]$ (3D M/GA)), Ni ([61, 84] (2D M/rGO), [61, 111] (3D M/GF and M/GA)), $\mathrm{Cu}[101], \mathrm{Ag}[90], \mathrm{Au}[74,81,182,194]$ or bimetallic electrocatalysts ([43, 44, 49, $74,76,79-83,90,92,93,95,96,99-101,104,106,117,124-126,158,163,182,185,194$, 195] (2D M/rGO), [165, 201] (3D M/GA or M/GF)) then became the alternative as summarized in Table 3. These graphene-based electrocatalysts loaded with such metals showed much stronger electrochemical activity in the half-cell scaled MOR $([43,44,61,79-$ $84,98-104,115,117,119,126,158,163,185,194,195]$ (2D M/rGO), [61, 113, 201] (3D M/GF)), EOR ([37, 44, 49, 90, 92-95, 99-104, 106, 107, 124] (2D M/rGO), [111, 201] (3D $\mathrm{M} / \mathrm{GF}$ and M/GA)), butan-1-ol electrooxidation (butan-1-ol OR) [125], glucose electrooxidation (GOR) $([74,118,182](2 \mathrm{D} \mathrm{M} / \mathrm{rGO}),[165](3 \mathrm{D} \mathrm{M} / \mathrm{GA}))$ and formic acid electrooxidation (FOR) $[48,76,96-98,114,184]$ with strong tolerance towards $\mathrm{CO}$ poisoning. These graphene-based electrocatalysts also showed stronger activity than monometallic $\mathrm{Pt} / \mathrm{Graphene}$ in the MOR and EOR. This point was reflected from the high anodic peak current density recorded from the best output current density with values of $4972 \mathrm{~A} \mathrm{~g}^{-1}$ [43] or $252 \mathrm{~mA} \mathrm{~cm}^{-2}$ [104] in MOR, $2105.4 \mathrm{~A} \mathrm{~g}^{-1}$ [101] or $2219 \mathrm{~mA} \mathrm{~cm} \mathrm{~cm}^{-2}$ [99] in EOR, respectively, as well as the best $\mathrm{I}_{\mathrm{f}} / \mathrm{I}_{\mathrm{b}}$ ratio of 11.65 in MOR [101] and 5.45 in EOR [49] as summarized in Table 4. Besides MOR and EOR, butan-1-ol OR [125], FOR [48, 76, 96-98, 
$114,184]$, and GOR $[74,118,165,182]$ catalyzed by graphene-based catalysts were another focus in recent years. However, systematic study in such topic was less relative to MOR and EOR, and non-platinum and bimetallic $\mathrm{M} /$ graphene-based electrocatalysts were the mainstream in this topic $[48,74,76,96-98,114,118,125,165,182,184]$. The results showed that the $\mathrm{M} / \mathrm{rGO}$ - or $\mathrm{M} / \mathrm{GA}$-based electrocatalysts exhibited strong activity in the butan-1-ol OR (Maximum $\mathrm{J}_{\mathrm{f}}: 15.59 \mathrm{~mA} \mathrm{~cm}{ }^{-2}$ or $105.98 \mathrm{~mA} \mathrm{mg}^{-1}$ [125]), FOR (Maximum $\mathrm{J}_{\mathrm{f}}: 61.6 \mathrm{~mA}$ $\mathrm{cm}^{-2}$ [98] or $3390 \mathrm{~A} \mathrm{~g}^{-1}$ [184]), and GOR (Maximum $\mathrm{J}_{\mathrm{f}}: 16.2 \mathrm{~mA} \mathrm{~cm}^{-2}$ [182] or $0.056 \mathrm{~A} \mathrm{~g}^{-1}$ [118]) as listed in Table 4, but only a few reports focused on the stability of the electrocatalysts after prolonged servicing (500 to 1248 cycles) $[165,182]$.

Table 2. List of the monometallic Pt loaded graphene-based electrocatalysts in MOR and EOR.

\begin{tabular}{|c|c|c|c|c|c|c|c|}
\hline $\begin{array}{l}\text { Graphene } \\
\text { host }\end{array}$ & Pt precursor & $\begin{array}{l}\text { Oxidation } \\
\text { Type }\end{array}$ & $\begin{array}{ll}\mathrm{J}_{\mathrm{f}} & (\text { Area } \\
\text { basis }) & (\mathrm{mA} \\
\left.\mathrm{cm}^{-2}\right) & \end{array}$ & $\begin{array}{l}\mathrm{J}_{\mathrm{f}} \quad(\text { Mass } \\
\text { basis) } \\
\left.\mathrm{mg}^{-1}\right)\end{array}$ & $\mathrm{I}_{\mathrm{f}} / \mathrm{I}_{\mathrm{b}}$ & Year & Ref \\
\hline \multirow[t]{26}{*}{$2 \mathrm{D}$ rGO } & Pt NPs & MOR & $\mathrm{N} / \mathrm{A}$ & 199.6 & 1.1 & 2009 & [91] \\
\hline & Pt NPs & MOR & 30 & N/A & 0.8 & 2010 & [87] \\
\hline & Pt NPs & MOR & 7.4 & N/A & 1.8 & 2010 & [187] \\
\hline & Pt NPs & MOR & $\mathrm{N} / \mathrm{A}$ & 195 & 1.3 & 2010 & [188] \\
\hline & Pt particles & MOR & N/A & 590 & 1.0 & 2011 & {$[85]$} \\
\hline & Pt NPs & MOR & 2.5 & $\mathrm{~N} / \mathrm{A}$ & 1.9 & 2011 & {$[86]$} \\
\hline & $\mathrm{Pt} / \mathrm{NFs}$ & MOR & N/A & 523 & 1.2 & 2012 & {$[46]$} \\
\hline & $\mathrm{Pt} / \mathrm{Nf}$ & EOR & 2.3 & $\mathrm{~N} / \mathrm{A}$ & 1.1 & 2012 & [49] \\
\hline & $\mathrm{Pt} / \mathrm{ND}$ & MOR & N/A & 98.7 & 0.9 & 2012 & {$[88]$} \\
\hline & Pt NPs & MOR & 44.2 & N/A & 0.9 & 2012 & [89] \\
\hline & Pt NPs & MOR & 57.7 & $\mathrm{~N} / \mathrm{A}$ & 1.2 & 2012 & [89] \\
\hline & Pt NPs & MOR & 0.5 & N/A & 1.9 & 2013 & [102] \\
\hline & Pt cluster & MOR & 22.9 & N/A & 2.2 & 2013 & [159] \\
\hline & Pt particles & MOR & N/A & 2540 & 4.2 & 2014 & [43] \\
\hline & Pt NPs & MOR & 16.5 & N/A & 3.3 & 2014 & [79] \\
\hline & Pt NPs & MOR & 0.9 & $\mathrm{~N} / \mathrm{A}$ & 6.6 & 2014 & [82] \\
\hline & PtNPs & MOR & 1.7 & N/A & 5.6 & 2014 & [83] \\
\hline & $\mathrm{PtNF}$ & EOR & 9.1 & N/A & 1.0 & 2014 & [93] \\
\hline & Pt NPs & MOR & 23 & N/A & 3.6 & 2014 & [104] \\
\hline & Pt NPs & EOR & 12 & $\mathrm{~N} / \mathrm{A}$ & $\mathrm{N} / \mathrm{A}$ & 2014 & [104] \\
\hline & Pt NPs & EOR & N/A & 26.7 & 4.8 & 2014 & [106] \\
\hline & $\mathrm{Pt}$ NF & MOR & 1.8 & N/A & 2.5 & 2015 & [80] \\
\hline & Pt PNWs & MOR & N/A & 394 & 1.0 & 2015 & [117] \\
\hline & Pt NPs & MOR & 1.0 & N/A & 1.4 & 2015 & [158] \\
\hline & Pt NPs & GOR & N/A & 14 & $\mathrm{~N} / \mathrm{A}$ & 2016 & [74] \\
\hline & PtNPs & MOR & 5.1 & N/A & 3.8 & 2017 & [78] \\
\hline
\end{tabular}


3D HGF $\quad$ PtNPs $\quad$ MOR 8.2

$1.6 \quad \mathrm{~N} / \mathrm{A}$

$\mathrm{N} / \mathrm{A}$

4.2

2012

$2017[78]$

Table 3. List of common monometallic non-Pt and bimetallic graphene-based electrocatalysts and their structure.

\begin{tabular}{|c|c|c|c|}
\hline Catalyst type & $\mathrm{M} / \mathrm{GNs}$ & Graphene Structure & Ref \\
\hline \multirow[t]{7}{*}{ Monometallic } & $\mathrm{Ag} / \mathrm{rGO}$ & $2 \mathrm{D}$ & {$[90]$} \\
\hline & $\mathrm{Au} / \mathrm{rGO}$ & $2 \mathrm{D}$ & {$[81,182,194]$} \\
\hline & $\mathrm{Cu} / \mathrm{rGO}$ & $2 \mathrm{D}$ & {$[101]$} \\
\hline & $\begin{array}{l}\mathrm{Ni} / 2 \mathrm{D}-\mathrm{rGO}, \quad \mathrm{Ni} / \mathrm{rGO} \quad(\mathrm{Ar} \\
\text { protect })\end{array}$ & $2 \mathrm{D}$ & {$[61,84]$} \\
\hline & $\mathrm{Ni} / 3 \mathrm{D}-\mathrm{rGO}, \mathrm{Ni} / \mathrm{GA}$ & $3 \mathrm{D}$ & {$[61,111]$} \\
\hline & $\begin{array}{l}\mathrm{Pd} / \mathrm{rGO}, \quad \mathrm{PdNCs} / \mathrm{rGO}, \quad \text { FS- } \\
\mathrm{Pd} / \mathrm{rGO}, \mathrm{Pd} / \mathrm{rGONP}\end{array}$ & $2 \mathrm{D}$ & $\begin{array}{l}{[37,44,48,49,79-} \\
83,90,93-104, \\
106,114,115,118, \\
119,158,163,184, \\
194]\end{array}$ \\
\hline & $\mathrm{Pd} / \mathrm{GA}, \mathrm{Pd} / \mathrm{GA} / \mathrm{NF}$ & $3 \mathrm{D}$ & {$[113,164]$} \\
\hline \multirow[t]{15}{*}{ Bimetallic } & $\mathrm{Ag} / \mathrm{Au} / \mathrm{rGO}$ & $2 \mathrm{D}$ & {$[182]$} \\
\hline & $\mathrm{Au} / \mathrm{Ag} / \mathrm{rGO}$ & $2 \mathrm{D}$ & {$[182]$} \\
\hline & Pd-Ag/rGO, $\mathrm{Pd}_{70} \mathrm{Ag}_{30} @ \mathrm{rGO}$ & $2 \mathrm{D}$ & {$[90,95,100,125]$} \\
\hline & $\begin{array}{l}\mathrm{Au} @ \mathrm{Pd} / \mathrm{rGO}, \\
\mathrm{rGO}-\mathrm{PuPd} @ \mathrm{Pd}-\mathrm{Au} / \mathrm{rGO},\end{array}$ & $2 \mathrm{D}$ & {$[81,99,194]$} \\
\hline & $\begin{array}{l}\mathrm{Pd}_{1} \mathrm{Cu}_{1} / \mathrm{rGO}, \mathrm{Pd} / \mathrm{Cu} / \mathrm{rGO}, \quad \mathrm{Pd}- \\
\mathrm{Cu} / \mathrm{rGO}\end{array}$ & $2 \mathrm{D}$ & {$[76,92,101]$} \\
\hline & $\mathrm{PdIr} / \mathrm{rGO}$ & $2 \mathrm{D}$ & {$[44]$} \\
\hline & $\mathrm{PdNi}-\mathrm{NN} / \mathrm{rGO}$ & $2 \mathrm{D}$ & {$[96]$} \\
\hline & $\begin{array}{l}\mathrm{PdPt} / \mathrm{rGO}, \quad \mathrm{Pd} @ \mathrm{Pt} / \mathrm{rGO}, \\
\mathrm{PdPt} @ \mathrm{Pt} / \mathrm{rGO}, \\
\mathrm{Pt}_{60} \mathrm{Pd}_{40} / \mathrm{rGONP}\end{array}$ & $2 \mathrm{D}$ & $\begin{array}{l}{[43,49,79,80,82,} \\
83,93,104,106, \\
107,126,158,195]\end{array}$ \\
\hline & $\mathrm{Pd}_{1} \mathrm{Pt}_{1.03} / \mathrm{GA} / \mathrm{NF}$ & $3 \mathrm{D}$ & {$[165,202]$} \\
\hline & $\mathrm{PdRu} / \mathrm{rGO}$ & $2 \mathrm{D}$ & {$[44,163]$} \\
\hline & $\mathrm{PdSn} / \mathrm{rGO}$ & $2 \mathrm{D}$ & {$[44]$} \\
\hline & $\mathrm{Pt}-\mathrm{Co} / \mathrm{rGO}$ & $2 \mathrm{D}$ & [185] \\
\hline & $\mathrm{Pt}_{75.4} \mathrm{Cu}_{24.6}$ Alloy/rGO & $2 \mathrm{D}$ & {$[124]$} \\
\hline & PtIr PNWs/rGO & $2 \mathrm{D}$ & {$[117]$} \\
\hline & $\mathrm{PtRu} / \mathrm{GF}$ & $3 \mathrm{D}$ & {$[201]$} \\
\hline
\end{tabular}

Table 4. List of the monometallic non-Pt and bimetallic graphene-based electrocatalyst performance in MOR, EOR, FOR and GOR.

\begin{tabular}{|c|c|c|c|c|c|c|c|}
\hline $\begin{array}{l}\text { Metal } \\
\text { catalyst }\end{array}$ & $\begin{array}{l}\text { Metal precursor } \\
\text { /Graphene host }\end{array}$ & $\begin{array}{l}\text { Type of } \\
\text { oxidation }\end{array}$ & $\begin{array}{l}\mathrm{J}_{\mathrm{f}} \quad(\text { Area } \\
\text { basis) }(\mathrm{mA} \\
\left.\mathrm{cm}^{-2}\right)\end{array}$ & $\begin{array}{lr}\mathrm{J}_{\mathrm{f}} & \text { (Mass } \\
\text { basis) } & (\mathrm{mA} \\
\left.\mathrm{mg}^{-1}\right) & \\
\end{array}$ & $\mathrm{I}_{\mathrm{f}} / \mathrm{I}_{\mathrm{b}}$ & Year & Ref \\
\hline \multirow{6}{*}{$\begin{array}{l}\text { Mono- } \\
\text { metallic }\end{array}$} & $\mathrm{Ag} / \mathrm{rGO}$ & EOR & 0 & N/A & $\mathrm{N} / \mathrm{A}$ & 2012 & [90] \\
\hline & $\mathrm{Au} / \mathrm{rGO}$ & MOR & 0.1 & N/A & N/A & 2014 & [81] \\
\hline & $\mathrm{Au} / \mathrm{rGO}$ & MOR & 0 & N/A & N/A & 2014 & [194] \\
\hline & $\mathrm{Au} / \mathrm{rGO}$ & GOR & 7.4 & N/A & N/A & 2014 & {$[182]$} \\
\hline & $\mathrm{Au} / \mathrm{rGO}$ & GOR & N/A & 27 & N/A & 2016 & [74] \\
\hline & $\mathrm{Cu} / \mathrm{rGO}$ & MOR & $\mathrm{N} / \mathrm{A}$ & 0 & N/A & 2015 & [101] \\
\hline
\end{tabular}




\begin{tabular}{|c|c|c|c|c|c|c|c|}
\hline $\begin{array}{l}\mathrm{Ni} / \mathrm{rGO}(\mathrm{Ar} \\
\left.\text { protect, } 500^{\circ} \mathrm{C}\right)\end{array}$ & MOR & 20 & & N/A & N/A & 2012 & {$[84]$} \\
\hline $\mathrm{Ni} / \mathrm{GA}$ & EOR & 16.3 & & N/A & N/A & 2013 & [111] \\
\hline $\mathrm{Ni} / 3 \mathrm{D}-\mathrm{G}$ & MOR & 62 & & N/A & N/A & 2017 & {$[61]$} \\
\hline $\mathrm{Pd}(10 \mathrm{~nm}) / \mathrm{rGO}$ & FOR & $\mathrm{N} / \mathrm{A}$ & & 210 & N/A & 2011 & {$[48]$} \\
\hline $\mathrm{Pd}(3 \mathrm{~nm}) / \mathrm{rGO}$ & FOR & $\mathrm{N} / \mathrm{A}$ & & 300 & N/A & 2011 & {$[48]$} \\
\hline $\mathrm{Pd} / \mathrm{rGO}$ & FOR & $\mathrm{N} / \mathrm{A}$ & & 213 & N/A & 2011 & [114] \\
\hline AP-Pd/rGO & FOR & $\mathrm{N} / \mathrm{A}$ & & 466.3 & N/A & 2011 & [114] \\
\hline $\mathrm{Pd}$ NPs/rGO & EOR & 46.7 & & N/A & 0.9 & 2012 & [37] \\
\hline $\mathrm{Pd} / \mathrm{rGO}$ & EOR & 0.56 & & N/A & 4.0 & 2012 & [49] \\
\hline $\mathrm{Pd} / \mathrm{rGO}$ & EOR & 22 & & N/A & 0.9 & 2012 & [90] \\
\hline $\mathrm{Pd} / \mathrm{LDrGO}$ & MOR & 27.6 & & N/A & 6.6 & 2012 & [98] \\
\hline $\mathrm{Pd} / \mathrm{rGO}$ & MOR & 15.0 & & N/A & 4.5 & 2012 & [98] \\
\hline $\mathrm{Pd} / \mathrm{LDrGO}$ & FOR & 61.6 & & N/A & N/A & 2012 & [98] \\
\hline $\mathrm{Pd} / \mathrm{rGO}$ & FOR & 34.6 & & $\mathrm{~N} / \mathrm{A}$ & N/A & 2012 & {$[98]$} \\
\hline $\mathrm{Pd} / \mathrm{rGO}$ & MOR & 360.8 & & N/A & 4.3 & 2013 & [99] \\
\hline $\mathrm{Pd} / \mathrm{rGO}$ & EOR & 897.6 & & N/A & 1.2 & 2013 & [99] \\
\hline $\mathrm{Pd} / \mathrm{rGO}$ & MOR & 0.8 & & N/A & 16.7 & 2013 & [102] \\
\hline $\mathrm{Pd} / \mathrm{rGO}$ & EOR & 3.8 & & N/A & 1.2 & 2013 & [102] \\
\hline $\mathrm{Pd} / \mathrm{rGO}$ & MOR & $\mathrm{N} / \mathrm{A}$ & & 522 & 6.1 & 2013 & [115] \\
\hline FS-Pd/rGO & GOR & $\mathrm{N} / \mathrm{A}$ & & 0.056 & N/A & 2013 & [118] \\
\hline $\mathrm{PdnD} / \mathrm{rGO}$ & MOR & 0.5 & & N/A & 7.5 & 2013 & [119] \\
\hline $\mathrm{PdnS} / \mathrm{rGO}$ & MOR & 0.1 & & N/A & 1.7 & 2013 & [119] \\
\hline $40 \% \mathrm{Pd} / \mathrm{rGO}$ & MOR & 47 & & N/A & 5.2 & 2013 & [163] \\
\hline $\mathrm{Pd} / \mathrm{rGO}$ & MOR & 96.1 & & N/A & 1.9 & 2014 & [79] \\
\hline $\mathrm{Pd} / \mathrm{rGO}$ & MOR & 0.2 & & N/A & N/A & 2014 & [81] \\
\hline $\mathrm{Pd} / \mathrm{rGO}$ & MOR & 0.4 & & N/A & 5.7 & 2014 & [82] \\
\hline PdNPs/rGO & MOR & 1.2 & & N/A & 3.2 & 2014 & {$[83]$} \\
\hline $\mathrm{PdNP} / \mathrm{rGO}$ & EOR & 7.5 & & N/A & 1.0 & 2014 & [93] \\
\hline $\mathrm{Pd} / \mathrm{rGO}$ & FOR & 3.2 & & N/A & N/A & 2014 & [97] \\
\hline $\mathrm{Pd} / \mathrm{rGO}$ & MOR & $\mathrm{N} / \mathrm{A}$ & & 311 & 1.4 & 2014 & {$[100]$} \\
\hline $\mathrm{Pd} / \mathrm{rGO}$ & EOR & $\mathrm{N} / \mathrm{A}$ & & 835 & 1.2 & 2014 & {$[100]$} \\
\hline $\mathrm{Pd} / \mathrm{rGO}$ & MOR & 0.4 & & 0.9 & 4.2 & 2014 & {$[103]$} \\
\hline $\mathrm{Pd} / \mathrm{rGO}$ & EOR & 1.5 & & 3.4 & 1.4 & 2014 & [103] \\
\hline $\mathrm{Pd} / \mathrm{rGO}$ & MOR & 22 & & N/A & 1.7 & 2014 & [104] \\
\hline $\mathrm{Pd} / \mathrm{rGO}$ & EOR & 23 & & N/A & 0.6 & 2014 & {$[104]$} \\
\hline $\mathrm{Pd} / \mathrm{rGO}$ & EOR & $\mathrm{N} / \mathrm{A}$ & & 13.7 & N/A & 2014 & {$[106]$} \\
\hline $\mathrm{Pd} / \mathrm{GA} / \mathrm{NF}$ & MOR & $\mathrm{N} / \mathrm{A}$ & & 798.8 & 3.1 & 2014 & [164] \\
\hline $\mathrm{Pd} / \mathrm{GA} / \mathrm{NF}$ & EOR & $\mathrm{N} / \mathrm{A}$ & & 874 & 2.9 & 2014 & [164] \\
\hline $\mathrm{Pd} / \mathrm{rGO}$ & MOR & 12.5 & & N/A & 2.8 & 2014 & [194] \\
\hline $\mathrm{Pd} / \mathrm{rGO}$ & MOR & $\begin{array}{l}0.1 \\
\left.\mathrm{~m}^{-2}\right)\end{array}$ & $(\mathrm{mA}$ & N/A & 2.1 & 2015 & [44] \\
\hline $\mathrm{Pd} / \mathrm{rGO}$ & EOR & $\begin{array}{l}0.3 \\
\left.\mathrm{~m}^{-2}\right)\end{array}$ & $(\mathrm{mA}$ & N/A & 1.4 & 2015 & {$[44]$} \\
\hline $\mathrm{Pd} \mathrm{NF} / \mathrm{rGO}$ & MOR & 1.9 & & N/A & 2.7 & 2015 & {$[80]$} \\
\hline $\mathrm{PdNCs} / \mathrm{rGO}$ & EOR & $\mathrm{N} / \mathrm{A}$ & & 429.8 & 1.8 & 2015 & [94] \\
\hline $\mathrm{Pd} / \mathrm{rGO}$ & EOR & 6.5 & & N/A & 0.8 & 2015 & [95] \\
\hline $\mathrm{Pd} / \mathrm{rGO}$ & FOR & $\mathrm{N} / \mathrm{A}$ & & 308.4 & N/A & 2015 & {$[96]$} \\
\hline
\end{tabular}




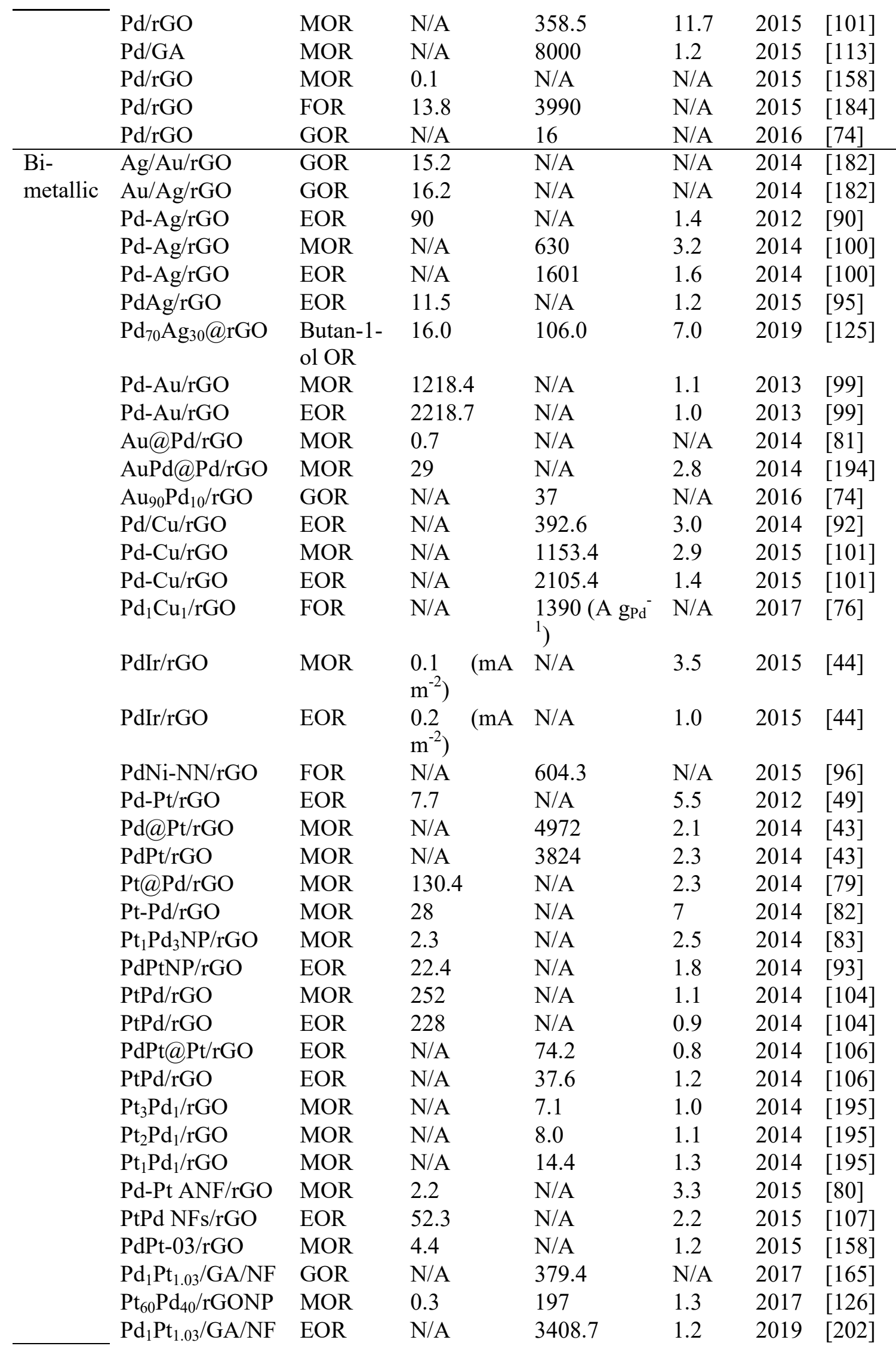




\begin{tabular}{|c|c|c|c|c|c|c|c|}
\hline $\begin{array}{l}40 \% \mathrm{Pd}- \\
5 \% \mathrm{Ru} / \mathrm{rGO}\end{array}$ & MOR & 118 & & N/A & 3.1 & 2013 & [163] \\
\hline $\mathrm{PdRu} / \mathrm{rGO}$ & MOR & $\begin{array}{l}0.2 \\
\left.\mathrm{~m}^{-2}\right)\end{array}$ & $(\mathrm{mA}$ & N/A & 2.4 & 2015 & {$[44]$} \\
\hline $\mathrm{PdRu} / \mathrm{rGO}$ & EOR & $\begin{array}{l}0.5 \\
\left.\mathrm{~m}^{-2}\right)\end{array}$ & $(\mathrm{mA}$ & N/A & 1.4 & 2015 & {$[44]$} \\
\hline $\mathrm{PdSn} / \mathrm{rGO}$ & MOR & $\begin{array}{l}0.1 \\
\left.m^{-2}\right)\end{array}$ & $(\mathrm{mA}$ & N/A & 2 & 2015 & {$[44]$} \\
\hline $\mathrm{PdSn} / \mathrm{rGO}$ & EOR & $\begin{array}{l}0.4 \\
\left.m^{-2}\right)\end{array}$ & $(\mathrm{mA}$ & N/A & 1.6 & 2015 & {$[44]$} \\
\hline $\mathrm{Pt}-\mathrm{Co} / \mathrm{rGO}$ & MOR & 12.3 & & N/A & 2.3 & 2014 & [185] \\
\hline $\begin{array}{l}\mathrm{Pt}_{75.4} \mathrm{Cu}_{24.6} \\
\text { Alloy/rGO }\end{array}$ & EOR & 12.3 & & 2.9 & 1.3 & 2018 & [124] \\
\hline $\begin{array}{l}\mathrm{Pt}_{68.2} \mathrm{Cu}_{31.8} \\
\text { Alloy/rGO }\end{array}$ & EOR & 11.5 & & 2.30 & 1.4 & 2018 & [124] \\
\hline PtIr PNWs/rGO & MOR & N/A & & 543 & 1.1 & 2015 & [117] \\
\hline $\mathrm{PtRu} / 3 \mathrm{D}$ GF & MOR & 109.3 & & N/A & 1.1 & 2014 & [201] \\
\hline $\mathrm{PtRu} / 3 \mathrm{D} \mathrm{GF}$ & EOR & 79.6 & & N/A & 1 & 2014 & [201] \\
\hline
\end{tabular}

Up to date, most of the graphene-based electrocatalyst research was done via the fabrication on a secondary substrate platform, such as a glassy carbon electrode. These works involved the use of binding or stabilization agents like Nafion solution with ultrasonic treatment for the catalyst ink production. Such a technique has the adverse effect of destroying the graphene nanosheets structure; this is especially obvious for $3 \mathrm{D}$ graphene nanostructures. The use of a conductive binder also results in the reduction of the catalytic active surface by the binder. To maximize the activity, the use of a binder-free graphene electrode has become an alternative in recent research. Binder-free graphene electrode has several strengths over the traditional electrode, especially the following. (1) The specific capacity and conductivity of the electrode can be increased when compared to those requiring the use of non-conductive and active binders. (2) Electron transfer inside the electrode can be strengthened with the help of interconnected or stacked graphene nanosheet throughout the self-assembly, which forms a strong conductive and robust network. (3) Effective contact between the electrolyte and the electrode interface due to the large specific surface area of the electrode results in the reduction of the diffusion resistance [29]. With reference to the 
binder-free pure GA/NF synthesis for direct supercapacitor electrode [32, 33], a metal-loaded GA/NF direct electrode was then developed by the same reaction pathway $[164,165,202-$ 204]. The results showed that strong activity was recorded in MOR (Maximum $\mathrm{J}_{\mathrm{f}}=798.8 \mathrm{~A} \mathrm{~g}^{-}$ ${ }^{1}$ ) and EOR (Maximum $\mathrm{J}_{\mathrm{f}}=862 \mathrm{~A} \mathrm{~g}^{-1}$ ) for binder-free Pd/GA/NF electrode [164]; EOR (Maximum $\left.\mathrm{J}_{\mathrm{f}}=3408.7 \mathrm{~A} \mathrm{~g}^{-1}\right)$ [202] and $\operatorname{GOR}\left(\mathrm{J}_{\mathrm{a} 2}=379.4 \mathrm{~A} \mathrm{~g}^{-1}\right)$ [165] for binder-free $\mathrm{Pd}_{1} \mathrm{Pt}_{\mathrm{x}} / \mathrm{GA} / \mathrm{NF}$ electrode, respectively, as shown in Table 4. Such a finding was outstanding relative to those fabricated by traditional electrode preparation techniques with high stability of 150 cycles over 1248 cycles in the prolonged GOR, and 100-500 cycles over 1456 cycles in the prolonged EOR, respectively $[164,165,202]$. Relative to the basic half-cell scaled electrode analysis mentioned previously, systematic research of the graphene-based electrocatalyst in the fuel cell unit or battery scale was relatively little [7, 203, 204]. Types of fuel cell unit covered were also limited to the direct ethanol fuel cell (DEFC) [203] and direct glucose fuel cell (DGFC) units [204]; the results showed that the output power density and current density were higher than the existing non-graphene catalyst-driven DEFC and DGFC units. More importantly, the binder-free $\mathrm{Pd}_{1} \mathrm{Pt}_{\mathrm{x}} / \mathrm{GA} / \mathrm{NF}$ electrode plate driven $\mathrm{DEFC}$ and DGFC units exhibited stronger cell unit activity than the Nafion-modified $\mathrm{Pd}_{1} \mathrm{Pt}_{\mathrm{x}} / \mathrm{GA} / \mathrm{NF}$ plate in the same DEFC (29.4\% of binder-free) and DGFC (66.5\% of binder-free) under identical fuel combination and working environment [203, 204].

\subsubsection{ORR catalysts for hydrogen fuel cells or metal-air batteries}

Hydrogen fuel cells and metal-air batteries are operated by ORR at cathode and hydrogen oxidation reaction (HOR) at the anode, but ORR was the major focus of graphene-based electrocatalyst-driven hydrogen fuel cell and metal-air battery research throughout the past decade. M/rGO (M=Pd, Pt, Au-Pd, Pd-Pt, PdPt@Pt Pt-Ag) [72, 73, 75, 77, 105-107, 109, 110, $118,122,158,194], \mathrm{MO}_{\mathrm{x}} / \mathrm{rGO}[7,127]$, and metal-free foreign element-doped graphene- 
based materials $[56,144,149,151,152,154,169,205]$ were commonly studied targets in either acidic $\left(\mathrm{HClO}_{4}\right.$ and $\left.\mathrm{H}_{2} \mathrm{SO}_{4}\right)[72,73,77,106,107,109,110,118,122,158,194]$ or basic $(\mathrm{NaOH}, \mathrm{KOH})[7,56,75,105,144]$ media. These catalysts showed stable activity in the ORR as reflected in their high current density output. An example is the result obtained from Wang et al.[7] using $\mathrm{BN}$-codoped graphene nanomesh $/ \mathrm{Co}_{3} \mathrm{O}_{4}$ catalyst in the $\mathrm{KOH}$ solution (see Figure 10). Some research groups also investigated the crossover effect towards the ORR performance of the cathodic electrode in the presence of alcohol (e.g. methanol) [7, 56, 73, $105,144]$. The results showed that the graphene-based ORR catalysts had strong tolerance towards the methanol added to the cathode area compared to commercial $\mathrm{Pt} / \mathrm{C}$ cathode, which was illustrated from the representative results demonstrated by a few research groups, including Wang's group in the Zn-air battery research using BN-codoped graphene nanomesh $/ \mathrm{Co}_{3} \mathrm{O}_{4}$ catalyst in $\mathrm{KOH}$ [7], Xie's group using PtNCs@NGA catalyzed ORR in $\mathrm{H}_{2} \mathrm{SO}_{4}$ [73] and Lv's group using Pd-Cu NCs/rGO catalyzed ORR in $\mathrm{KOH}$ [105] as shown in Figures 10-12, respectively.
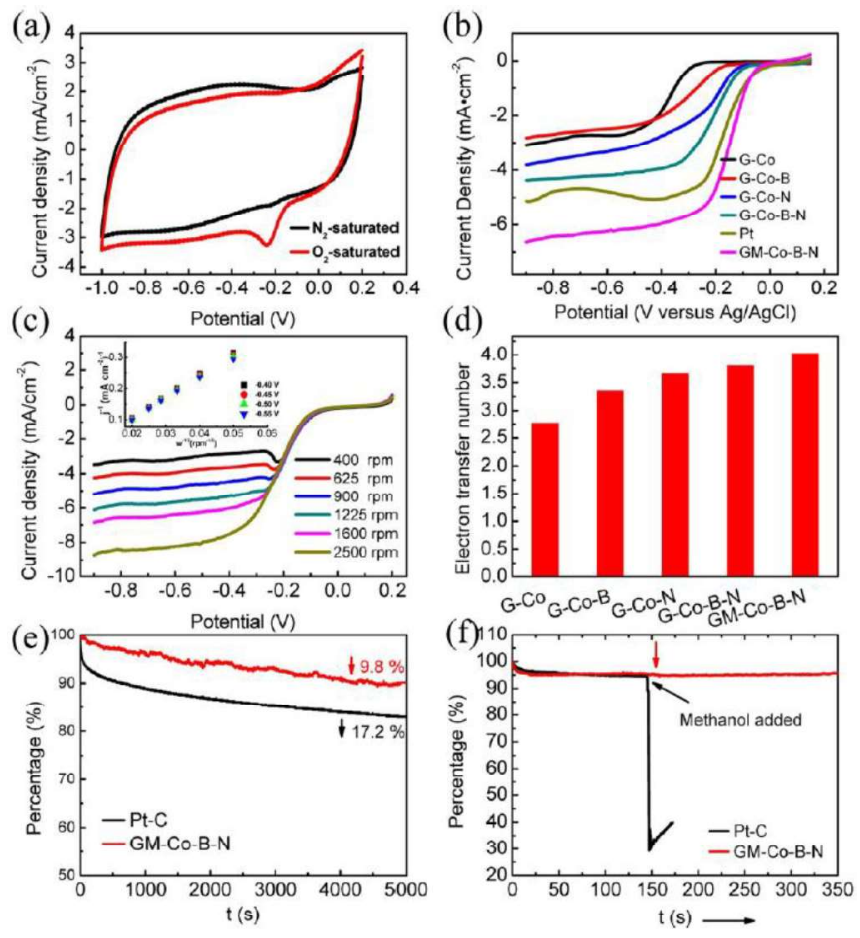
Fig 10. (a) CV curves of GM-Co-B-N in $\mathrm{O}_{2}$ - and $\mathrm{N}_{2}$-saturated electrolyte. (b) Polarization curves of G-Co, G-Co-B, G-Co-N, G-Co-B-N, and GM-Co-B-N at 1600 rpm rotation speed in $\mathrm{O}_{2}$-saturated electrolyte. (c) LSV curves of GM-Co-B-N in diff erent rotation speeds in the range from 400 to $2500 \mathrm{rpm}$, inset shows the $\mathrm{K}-\mathrm{L}$ plot of GM-Co-B-N. (d) Electron transfer numbers of catalyst samples. (e) The durability of electrodes and (f) current density loss-time CA responses of GM-Co-B-N and $\mathrm{Pt} / \mathrm{C}$ electrodes at $-0.3 \mathrm{~V}$ in oxygen-saturated electrolyte at rotating speed of $1200 \mathrm{rpm}$. The arrow indicates the addition of $3 \mathrm{M}$ methanol into the electrochemical cells.[7] (ACS)
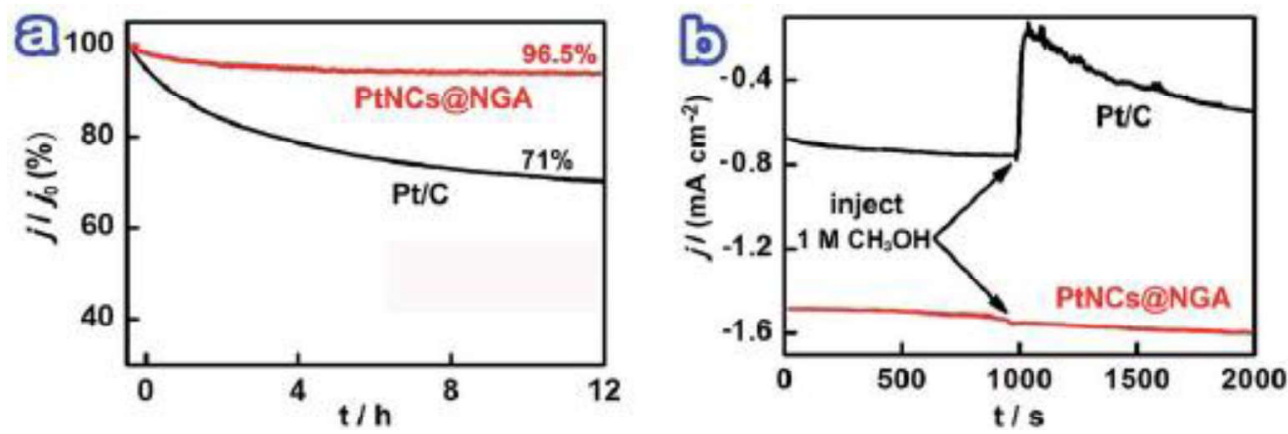

Fig 11. (a) Chronoamperometric responses of the PtNCs@NGA and commercial Pt/C catalysts for $12 \mathrm{~h}$ in an $\mathrm{O}_{2}$-saturated aqueous solution of $0.5 \mathrm{M} \mathrm{H}_{2} \mathrm{SO}_{4}$. (b) Chronoamperometric responses of the PtNCs@NGA and Pt/C catalysts in an $\mathrm{O}_{2}$-saturated aqueous solution of $0.5 \mathrm{M} \mathrm{H}_{2} \mathrm{SO}_{4}$ to injection of $1 \mathrm{M}$ methanol.[73] (RSC) 

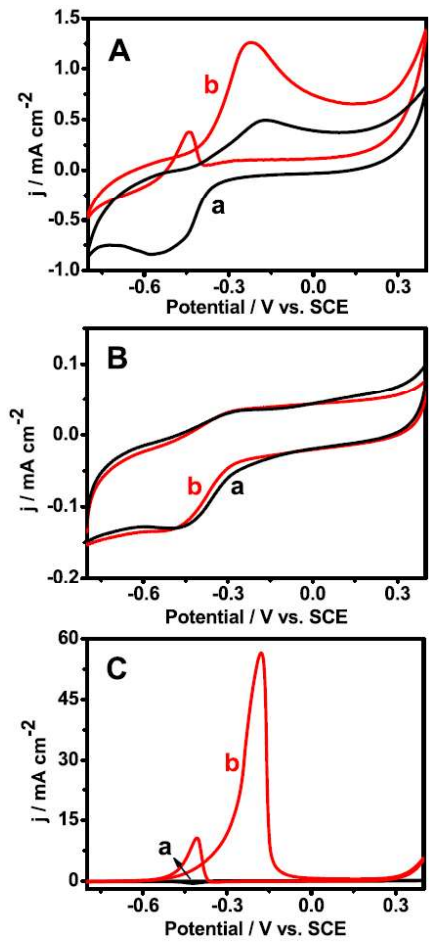

Fig 12. CVs of Pd-Cu NCs/RGOs (A), RGOs (B), and Pd black (C) modified electrodes without (curve a) and with (curve b) $3.0 \mathrm{M}$ methanol in $0.1 \mathrm{M} \mathrm{KOH}$ at the scan rate of $50 \mathrm{mV}$ $\mathrm{s}^{-1} \cdot[105]($ Elsevier B. V.)

Same as alcohol fuel cell research, the demonstration of graphene catalyst-driven hydrogen fuel cell or metal-air battery in the device scale was rare. A representative example was the demonstration of $\mathrm{MO}_{\mathrm{x}} / \mathrm{rGO}$ as the electrode materials for the zinc-air battery ( $\mathrm{Zn}$-air battery), with $\mathrm{BN}$-codoped graphene nanomesh $/ \mathrm{Co}_{3} \mathrm{O}_{4}$ chosen as the cathodic material by Wang's group [7]. The battery showed comparable activity to the commercial $\mathrm{Pt} / \mathrm{C}$ cathode-driven Zn-air battery after prolonged servicing for $10 \mathrm{~h}$, which is reflected by the battery performance in a single cycle prolonged operation as shown in Figure 13 [7]. 

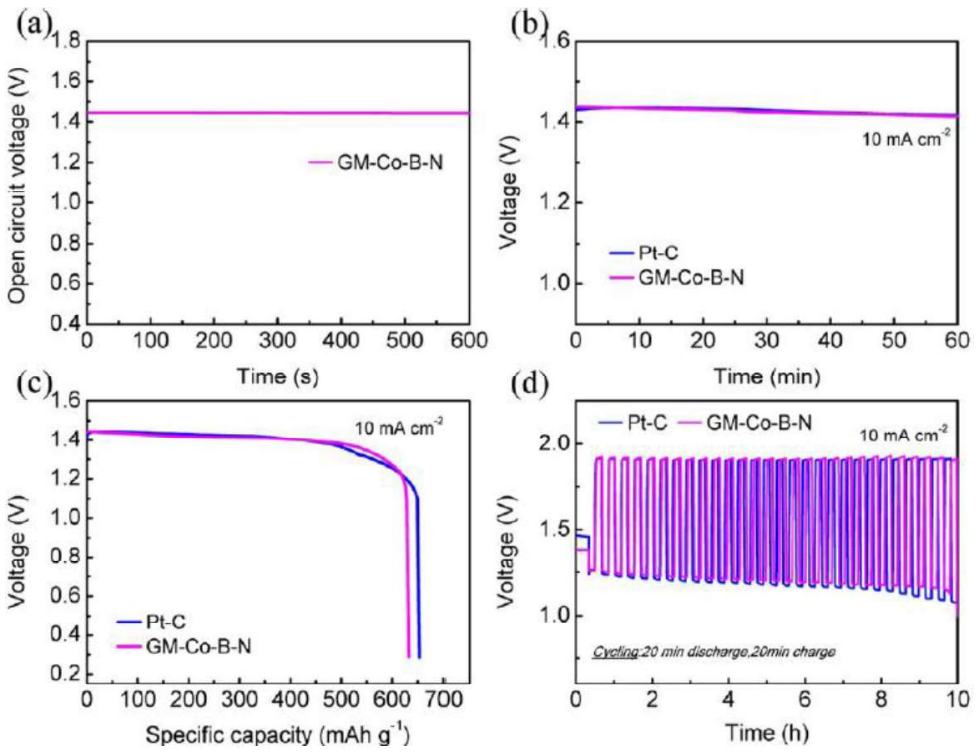

Fig 13. (a) Open-circuit voltage of GM-Co-B-N-based Zn-air battery. (b) Typical galvanostatic discharge curves of Zn-air batteries with GM-Co-B-N and Pt/C as cathode catalysts at $10 \mathrm{~mA} \mathrm{~cm}^{-2}$ current densities. (c) Long-term galvanostatic discharge curves of Zn-air batteries and (d) galvanostatic discharge-charge cycling curves, current density $10 \mathrm{~mA}$ $\mathrm{cm}^{-2}, 20 \mathrm{~min}$ for each state.[7] (ACS)

\subsection{Lithium ion and lithium metal batteries}

Besides the huge effort on the graphene-based fuel cell catalyst research, the use of graphene in lithium-ion battery (LIBs), lithium-air (Li-Air) battery and supercapacitor research has also been a popular topic in recent years. It is because graphene exhibited strong electrical conductivity with their large specific surface area, which is beneficial for the charge transfer in the LIB and Li-air battery operation $[8,9,21,126,128,130,137,141-143,155$, 161, 206-210]. Pure graphene [206], metal loaded graphene [126], metal oxide-loaded graphene $[8,128,130,137,141-143,161,207,209,210]$, and the metal-free foreign elements (B, N, S)-doped graphene [9, 21, 155, 208] (2D rGO [21, 126, 128, 130, 137, 141$143,155,206-210]$ and 3D GA $[8,9,161])$ were commonly used materials for LIBs and Li- 
Air battery anode preparation. They showed high specific capacities in the LIBs and Li-Air battery discharge step in the range of 0.06 to $15000\left(\mathrm{mAh} \mathrm{g}^{-1}\right)[8,9,21,126,128,130,137$, $141-143,155,161,206-210]$, with high stability and a large number of operation cycles (2010000 cycles) in the non-aqueous $\mathrm{Li}^{+}$-based (Lithium bis(trifluoromethanesulfonyl) imide (LITFSI), lithium hexafluorophosphate $\left(\mathrm{LiPF}_{6}\right)$, Lithium triflate $\left(\mathrm{LiCF}_{3} \mathrm{SO}_{3}\right)$, or Lithium nitrate(V) $\left.\left(\mathrm{LiNO}_{3}\right)\right)$ electrolyte solution as summarized in Table $5[8,9,21,126,128,130$, 137, 141-143, 155, 161, 206-210]. Among them, Meng et al.[9] developed the most stable graphene anodic materials for LIBs as the discharge specific capacity could keep stable for 400 cycles at a current density of $500 \mathrm{mAh} \mathrm{g}^{-1}$, and 2-3 times stronger discharge activity than the pure graphene aerogel as shown in Figure 14. Sun et al.[8] achieved even stronger stability via the use of $\mathrm{Nb}_{2} \mathrm{O}_{5}$-loaded holey graphene framework $\left(\mathrm{Nb}_{2} \mathrm{O}_{5} / \mathrm{HGF}-2.0\right)$ as LIBs anode, where the capacity retention achieved was $90 \%$ after 10000 -cycle operations [8]. Even though the specific discharge capacitance was not very high $\left(\sim 150 \mathrm{mAh} \mathrm{g}^{-1}\right)$ compared to NGA developed by Meng's group (500 $\mathrm{mAh} \mathrm{g}^{-1}$ ) [9], such HGF anode was directly used without the assistance of binder and conductive additives, which showed the advanced point of binder-free free-standing bulk 3D graphene framework as direct electrode for LIBs or green energy storage devices [8]. This hypothesis was proved from the large difference in specific capacitance recorded in the control sample of $2 \mathrm{D} \mathrm{Nb} \mathrm{O}_{5} / \mathrm{G}\left(\sim 60 \mathrm{mAh} \mathrm{g}^{-1}\right)$ against the 3D $\mathrm{Nb}_{2} \mathrm{O}_{5} / \mathrm{HGF}-2.0\left(145 \mathrm{mAh} \mathrm{g}^{-1}\right)$ under the same $\mathrm{Nb}_{2} \mathrm{O}_{5}$ loading $\left(6 \mathrm{mg} \mathrm{cm}^{-1}\right)$ in the same electrochemical analysis.

Table 5. List of graphene-based LIBs, Li-Air battery electrode materials.

\begin{tabular}{lllllll}
\hline $\begin{array}{l}\text { (Catalyst } \\
\text { precursor and) } \\
\text { Graphene host }\end{array}$ & $\begin{array}{l}\text { Battery } \\
\text { Type }\end{array}$ & $\begin{array}{l}\text { Discharge } \\
\text { capacitance } \\
\left(\mathrm{mAh} \mathrm{g}^{-1}\right)\end{array}$ & $\begin{array}{l}\text { Charge } \\
\text { capacitance } \\
\left(\mathrm{mAh} \mathrm{g}^{-1}\right)\end{array}$ & $\begin{array}{l}\text { Cycle } \\
\text { (Depreciation) }\end{array}$ & Year & Ref \\
\hline N-Graphene & $\mathrm{LIBs}$ & 0.1 & $\mathrm{~N} / \mathrm{A}$ & $50(40 \%)$ & 2010 & {$[21]$} \\
$\mathrm{FGSs}$ & $\mathrm{Li-O} 2$ & 15000 & N/A & N/A & 2011 & {$[206]$} \\
$\alpha-\mathrm{MnO}_{2} / \mathrm{RGO}$ & $\mathrm{LIBs}$ & 154 & $\mathrm{~N} / \mathrm{A}$ & $20(0 \%)$ & 2012 & {$[210]$} \\
$\mathrm{TiO}_{2}(\mathrm{~B}) / \mathrm{rGO}$ & $\mathrm{LIBs}$ & 635 & 613 & $100(6.5 \%)$ & 2013 & {$[141]$} \\
$\mathrm{P} 25 /$ graphene & LIBs & 319 & 322 & $50(12.2 \%)$ & 2013 & {$[142]$}
\end{tabular}




\begin{tabular}{|c|c|c|c|c|c|c|}
\hline $\begin{array}{l}\text { Porous graphene- } \\
\mathrm{Co}_{3} \mathrm{O}_{4} @ \mathrm{C}\end{array}$ & LIBs & 780 & $\mathrm{~N} / \mathrm{A}$ & $100(0 \%)$ & 2013 & {$[207]$} \\
\hline $\begin{array}{l}\text { Porous graphene- } \\
\mathrm{Fe}_{3} \mathrm{O}_{4} @ \mathrm{C}\end{array}$ & LIBs & 1391 & $\mathrm{~N} / \mathrm{A}$ & $100(19.2 \%)$ & 2013 & {$[207]$} \\
\hline $\mathrm{ATN} / \mathrm{rGO}$ & LIBs & 298.1 & $\mathrm{~N} / \mathrm{A}$ & $200(13.9 \%)$ & 2014 & {$[130]$} \\
\hline N-GA & LIBs & 2602.4 & $\mathrm{~N} / \mathrm{A}$ & $100(79.4 \%)$ & 2015 & [9] \\
\hline $\mathrm{ZnO} / \mathrm{rGO}$ & LIBs & 1724 & $\mathrm{~N} / \mathrm{A}$ & $200(79.2 \%)$ & 2015 & {$[128]$} \\
\hline B-rGO & LIBs & 723 & 801 & $60(30 \%)$ & 2015 & {$[208]$} \\
\hline NS-rGO & $\mathrm{Li}-\mathrm{O}_{2}$ & 1000 & $\mathrm{~N} / \mathrm{A}$ & $40(\sim 0 \%)$ & 2016 & {$[155]$} \\
\hline $\mathrm{Nb}_{2} \mathrm{O}_{5} / \mathrm{HGF}-2.0$ & LIBs & 125 & $\mathrm{~N} / \mathrm{A}$ & $10000(10 \%)$ & 2017 & {$[8]$} \\
\hline $\mathrm{Pt}_{40} \mathrm{Pd}_{60}-\mathrm{rGONPs}$ & LI-O ${ }_{2}$ & 1000 & N/A & $80(\sim 0 \%)$ & 2017 & {$[126]$} \\
\hline PSCMnO & LIBs & 1072 & $\mathrm{~N} / \mathrm{A}$ & $\begin{array}{ll}500 & (6 \% \\
\text { increase }) & \end{array}$ & 2018 & {$[143]$} \\
\hline $\mathrm{Fe}_{3} \mathrm{O}_{4} \mathrm{NWs} / \mathrm{GA}$ & LIBs & 1296 & 905 & $100(30.6 \%)$ & 2018 & {$[161]$} \\
\hline $\mathrm{Co}_{3} \mathrm{O}_{4} / \mathrm{rGO}$ & LIBs & 47 & 42.7 & $100(\sim 0 \%)$ & 2019 & {$[137]$} \\
\hline $\mathrm{LTO} / \mathrm{HG}$ & LIBs & 117 & $\mathrm{~N} / \mathrm{A}$ & $1000(\sim 6.7 \%)$ & 2019 & [209] \\
\hline
\end{tabular}
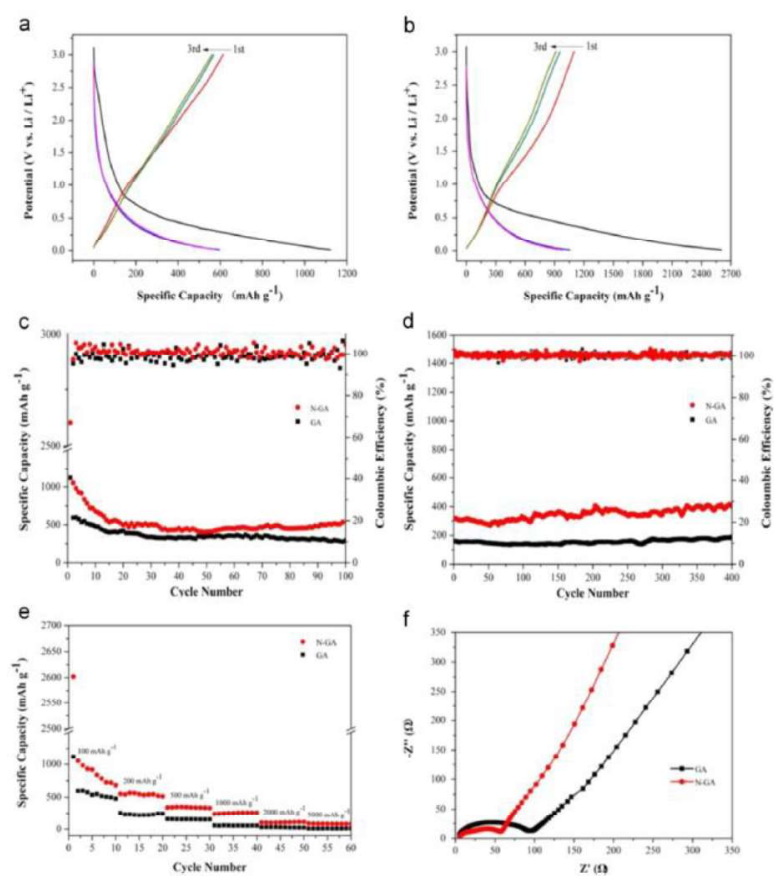

Fig 14. First three charge/discharge curves of GA (a) and N-GA (b) at $100 \mathrm{~mA} \mathrm{~g}^{-1}$; Cycling performances of GA and N-GA at $100 \mathrm{~mA} \mathrm{~g}^{-1}$ (c) and $500 \mathrm{~mA} \mathrm{~g}^{-1}$ (d); Rate capability at different current density (e) and Nyquist plots (f) of GA and N-GA.[9] (Elsevier B. V.)

The capability of the graphene products as anodic materials for device scale LIBs was an important index for the application; bulk cylindrically shaped 3D GAs products were 
especially suitable to fit into the conventional coin cells without treatment when compared to 2D graphene electrode materials [8,9]. Studies carried out by Sun [8] and Meng [9] showed that the $\mathrm{Nb}_{2} \mathrm{O}_{5} / \mathrm{HGF}$ and $\mathrm{N}$-doped GA can be directly mechanically assembled into traditional coin cells (CR 2016 and CR 2025) for the electrochemical analysis of the GA anodes without the use of binder and conducting additives [8,9]. Sun even showed that complicated fabrication treatment process with the use of additives was required when $2 \mathrm{D} \mathrm{Nb}_{2} \mathrm{O}_{5} / \mathrm{G}$ was used for the LIBs anode assembly [9]. Excellent electrochemical properties, as well as an easy and clean assembly process for the LIBs anode exhibited by $\mathrm{Nb}_{2} \mathrm{O}_{5} / \mathrm{HGF}$ against $2 \mathrm{D}$ $\mathrm{Nb}_{2} \mathrm{O}_{5} / \mathrm{G}$ mentioned above, clearly showed the strength of free-standing GA electrode for green energy.

\subsection{Supercapacitors}

Relatively, effort in supercapacitor research was on large, pure graphene $[32,33,57,58,62$, 67-71, 162, 167, 211-217], metal [121], metal hydroxide [116], layered double hydroxide [218-222], metal oxide $([23,69,129,131,132,134,136,160,168,190,196])$, foreign elements (B, N, S, or P)-doped pure graphene [145, 146, 148, 153, 156, 157, 223-225], and carbon nanotube [33]. These were the most commonly studied materials as summarized in Table 6 (2D graphene: ([129, 131-133, 136, 153, 168, 190, 196, 211, 212, 214, 215, 217-222, 224]), 3D graphene: $[23,32,33,42,57,58,62,65,67-71,116,121,134,145,146,148,150$, $156,157,160,162,213,216,223,225])$, which exhibited strong specific capacity in the range of 40-2100 ( $\left.\mathrm{F} \mathrm{g}^{-1}\right)$ with high stability of 20-15000 service cycles with low capacitance depreciation as listed in Table $7[23,32,33,42,57,58,62,65,67-71,116,121,129,131-134$, $136,145,146,148,150,153,156,157,160,162,168,190,196,211-225]$. In the ordinary supercapacitor electrode analysis, liquid- or gel-like electrolyte like $\mathrm{KOH}, \mathrm{NaOH}$ and $\mathrm{H}_{2} \mathrm{SO}_{4}$ was used in the supercapacitor electrode performance analysis $[23,32,33,57,58,62,67,69$ - 
$71,116,121,129,131,132,136,145,146,148,153,156,157,160,162,168,190,196,211-$ 225], and a few of the cases were carried out at $\mathrm{Na}_{2} \mathrm{SO}_{4}$ solution [68]. However, an investigation carried out by Yin et al. [134] was special in that the $\mathrm{GA} / \mathrm{TiO}_{2}$ electrode showed strong specific capacitance in simulated saline water $(0.1 \mathrm{M} \mathrm{NaCl}$ solution) with a range of 50-150 $\mathrm{F} \mathrm{g}^{-1}$ under different scanning rates $\left(5-1000 \mathrm{mV} \mathrm{s}^{-1}\right)$. However, it achieved almost zero retention in capacitance in the simulated saline water after 1000 cycles of operation in $0.1 \mathrm{M} \mathrm{NaCl}$ [134]. $\mathrm{GA} / \mathrm{TiO}_{2}$ electrode also showed strong desalination efficiency after reaching equilibrium at 200 s with strong regeneration efficiency over 10 cycles, which was much stronger than those achieved by pure GA and neat activated carbon. These excellent performances were reflected in Figure 15 [134]. Even though the low specific capacitance retention (4.9\%) of $\mathrm{Gr} / \mathrm{SnO}_{2}$ in $\mathrm{NaCl}$ solution was short lasting (50 cycles) from demonstration by El-Deen AG et al [133] in 2014, it still achieved 83\% salt removal efficiency from $\mathrm{NaCl}$ solution. Such demonstrations provided the possibility of using metal oxide/GA as the fresh water generation materials via the desalination of seawater using supercapacitor operation.

Table 6. List of graphene-based materials for supercapacitor electrode.

\begin{tabular}{|c|c|c|c|}
\hline $\begin{array}{l}\text { 2D graphene-based } \\
\text { supercapacitor materials }\end{array}$ & Ref & $\begin{array}{l}\text { 3D graphene-based } \\
\text { supercapacitor materials }\end{array}$ & Ref \\
\hline Pure rGO & {$[211,214,215]$} & Pure GH & $\begin{array}{l}{[32,42,57,58,} \\
65,162,216]\end{array}$ \\
\hline RGON & [217] & Pure GA & $\begin{array}{l}{[32,33,42,57,} \\
62,67,134,213]\end{array}$ \\
\hline $\begin{array}{l}\text { Microwave exfoliated } \\
\text { GO (MEGO) }\end{array}$ & {$[212]$} & 3D RGO/Ni foam & {$[68]$} \\
\hline $\mathrm{Co}_{3} \mathrm{O}_{4} / \mathrm{rGO}-\mathrm{C}$ & {$[136]$} & GCA@NF & {$[33]$} \\
\hline Graphene- $\mathrm{MnO}_{2}$ & {$[129]$} & 3D-NiGO & [69] \\
\hline $\mathrm{MnO}_{2} / 3 \mathrm{D} \mathrm{CGM}$ & [196] & $\mathrm{HrGO} / \mathrm{NF}$ & {$[70]$} \\
\hline $\mathrm{NF} / \mathrm{G} / \mathrm{MnO}_{2}$ & [168] & rGO@Ni foam & {$[71]$} \\
\hline $\mathrm{Gr} / \mathrm{SnO}_{2}$ & [133] & $\mathrm{MnO}_{2} / \mathrm{GF}$ & [23] \\
\hline $\mathrm{ZnO} / \mathrm{GNs}$ & {$[131,132,190]$} & $\begin{array}{l}\text { 3D-NiGOM } \quad\left(\mathrm{MnO}_{2}\right. \\
\text { nanowires })\end{array}$ & [69] \\
\hline $\begin{array}{l}\mathrm{Ni}^{2+} / \mathrm{Al}^{3+}(\mathrm{GNS} / \mathrm{LDH}) \\
\text { graphene/NiAl-LDH, } \\
\text { 3D-ARGON/NiAl-LDH }\end{array}$ & [218-220] & $\mathrm{Mn}_{3} \mathrm{O}_{4} / \mathrm{GAs}$ & {$[160]$} \\
\hline
\end{tabular}




\begin{tabular}{|c|c|c|c|}
\hline \multirow{2}{*}{\multicolumn{4}{|c|}{$\begin{array}{l}\text { Co-Al LDH-NS, }[221,222] \\
\text { GNS/CoAl-LDH }\end{array}$}} \\
\hline & & & \\
\hline $\mathrm{B} / \mathrm{GNs}$ & {$[153,224]$} & $\mathrm{Co} / \mathrm{GA}$ & {$[121]$} \\
\hline N/GNs & {$[224]$} & $\mathrm{GA} / \mathrm{TiO}_{2}$ & {$[134]$} \\
\hline $\mathrm{BCN} / \mathrm{GNs}$ & [224] & B-doped GA & [148] \\
\hline & & N-doped GA & {$[145,148]$} \\
\hline & & N-doped GH & {$[146]$} \\
\hline & & $\mathrm{NG} / \mathrm{NF}$ & {$[150]$} \\
\hline & & S-doped GA & {$[225]$} \\
\hline & & BN co-doped GA & [148] \\
\hline & & PS co-doped GA & [223] \\
\hline & & NS-GA & {$[157]$} \\
\hline & & SNG aerogel & {$[156]$} \\
\hline
\end{tabular}

Table 7. List of graphene-based supercapacitor electrode performance.

\begin{tabular}{|c|c|c|c|c|}
\hline (Precursor)/Graphene host & $\begin{array}{l}\text { Specific } \\
\text { capacitance }\left(\mathrm{F} \mathrm{g}^{-1}\right)\end{array}$ & Cycle (Depreciation) & Year & Ref \\
\hline SGH & 160 & $\mathrm{~N} / \mathrm{A}$ & 2010 & [216] \\
\hline Graphene aerogel & 128 & $\mathrm{~N} / \mathrm{A}$ & 2011 & [42] \\
\hline GH-Hz8 & 220 & $2000(8 \%)$ & 2011 & {$[58]$} \\
\hline SGHs & 240 & $\mathrm{~N} / \mathrm{A}$ & 2011 & {$[65]$} \\
\hline Nanomesh graphene & 255 & $2000(5.9 \%)$ & 2011 & [211] \\
\hline a-MEGO & 165 & $10000(3 \%)$ & 2011 & [212] \\
\hline $\mathrm{G}-\mathrm{Gel} / \mathrm{NF}$ & $41\left(\mathrm{mF} \mathrm{cm}^{-2}\right)$ & $10000(10 \%)$ & 2012 & [32] \\
\hline aG-O film & 120 & $2000(5 \%)$ & 2012 & [214] \\
\hline Porous PGNs & 154 & $5000(12 \%)$ & 2012 & [215] \\
\hline GA@NF & 366 & $2000\left(15 \%\left(2 \mathrm{~A} \mathrm{~g}^{-1}\right)\right)$ & 2013 & [33] \\
\hline rGH & 232 & $100(4 \%)$ & 2013 & [57] \\
\hline \multirow[t]{2}{*}{ RGOA } & $212(\mathrm{KOH})$ & $1000(\sim 5 \%, \mathrm{KOH})$ & 2013 & {$[62]$} \\
\hline & $279\left(\mathrm{H}_{2} \mathrm{SO}_{4}\right)$ & $1000\left(1.5 \%, \mathrm{H}_{2} \mathrm{SO}_{4}\right)$ & & \\
\hline G-gel@NF-2 & 152 & $2000(\sim 23 \%)$ & 2014 & {$[71]$} \\
\hline 3D-NiGO & 236 & $1000(5 \%)$ & 2015 & [69] \\
\hline $\mathrm{HrGO} / \mathrm{NF}$ & 334 & $1000(0.4 \%)$ & 2015 & {$[70]$} \\
\hline GO-RFA-P & 56 & $\mathrm{~N} / \mathrm{A}$ & 2015 & [213] \\
\hline FAG 400 & 442 & $1600(5 \%)$ & 2016 & [67] \\
\hline $\mathrm{RGO} / \mathrm{Ni}$ foam composite & 207 & $10000(2.6 \%)$ & 2016 & [68] \\
\hline RGON & 33 & $\mathrm{~N} / \mathrm{A}$ & 2017 & [217] \\
\hline GCA@NF & 207 & $2000\left(20 \%\left(10 \mathrm{~A} \mathrm{~g}^{-1}\right)\right)$ & 2013 & [33] \\
\hline PANI@3DGFs & 932 & $5000(29.8 \%)$ & 2015 & [162] \\
\hline B-GAs & 228 & $1000(0 \%)$ & 2012 & [148] \\
\hline BT-rGO & 448 & $3000(0 \%)$ & 2015 & [153] \\
\hline B-graphene & 83 & N/A & 2015 & [224] \\
\hline N-GAs & 190 & $1000(0 \%)$ & 2012 & [148] \\
\hline GN-GH & 190 & $4000(4.8 \%)$ & 2013 & [146] \\
\hline NGA & 223 & $2000(\sim 8 \%)$ & 2015 & [145] \\
\hline N-graphene & 111 & N/A & 2015 & [224] \\
\hline NG/NF & 223 & $3000(0 \%)$ & 2016 & [150] \\
\hline NS-GA-5 (GO:S=5:1) & 203 & $3000(10 \%)$ & 2018 & [157] \\
\hline SNG aerogel & 254 & $5000(16.5 \%)$ & 2018 & [156] \\
\hline BN-GAs & 239 & $1000(0 \%)$ & 2012 & [148] \\
\hline
\end{tabular}




\begin{tabular}{|c|c|c|c|c|}
\hline $\mathrm{BCN}$ graphene & 131 & $2000(2.5 \%)$ & 2015 & [224] \\
\hline SRGA & 446 & $1500(26.6 \%)$ & 2015 & [225] \\
\hline SP-AG & 381 & $10000(6.6 \%)$ & 2016 & [223] \\
\hline GNS/LDH & 782 & $200(22.6 \%$ Increase $)$ & 2011 & [218] \\
\hline Co-Al LDH-NS/GO & 880 & $2000(\sim 0 \%)$ & 2012 & [221] \\
\hline GNS/CoAl-LDH & 712 & $2000(19 \%)$ & 2012 & [222] \\
\hline Graphene/NiAl-LDH & 214 & $\begin{array}{l}250(9.3 \%) \\
1000(\sim 0 \%)\end{array}$ & 2013 & [219] \\
\hline 3D-ARGON/NiAl-LDH & 2713 & $5000(1.1 \%)$ & 2013 & [220] \\
\hline $\begin{array}{l}\text { Co(II) ion-modified r-GO } \\
\text { aerogels }\end{array}$ & 387 & $1000(\sim 0 \%)$ & 2014 & [121] \\
\hline $\mathrm{Co}_{3} \mathrm{O}_{4} / \mathrm{rGO}-\mathrm{C}$ & 709 & $6000(8.8 \%)$ & 2019 & [136] \\
\hline $\begin{array}{l}\text { Graphene- } \mathrm{MnO}_{2} \\
\text { composite }\end{array}$ & 310 & $15000(4.6 \%)$ & 2010 & [129] \\
\hline $\mathrm{MnO}_{2} / \mathrm{GF}$ & 670 & $\mathrm{~N} / \mathrm{A}$ & 2014 & {$[23]$} \\
\hline 3D-NiGOM & 1186 & $1000(22 \%)$ & 2015 & [69] \\
\hline $3 \mathrm{D} \mathrm{CMG} / \mathrm{MnO}_{2}$ & 421 & $1000(3 \%)$ & 2015 & [196] \\
\hline $\mathrm{NF} / \mathrm{G} / \mathrm{MnO}_{2}$ & $723\left(\mathrm{~F} \mathrm{~cm}^{-2}\right)$ & $2300(2.4 \%)$ & 2017 & [168] \\
\hline $\mathrm{Mn}_{3} \mathrm{O}_{4} / \mathrm{GAs}$ & 162 & $5000(11.2 \%)$ & 2019 & [160] \\
\hline $\mathrm{Ni}(\mathrm{OH})_{2} \cdot(\mathrm{GN})$ & 533 & $2000(\sim 0 \%)$ & 2013 & [116] \\
\hline $\mathrm{Gr} / \mathrm{SnO}_{2}$ & $323(\mathrm{NaCl})$ & $50(4.9 \%, \mathrm{NaCl})$ & 2014 & [133] \\
\hline $\mathrm{GA} / \mathrm{TiO}_{2}$ & $\begin{array}{l}143(\mathrm{NaCl}) \\
245(\mathrm{KOH})\end{array}$ & $1000(\sim 0 \%, \mathrm{NaCl})$ & 2013 & [134] \\
\hline $\mathrm{ZnO} / \mathrm{rGO}$ composites & 308 & $1500(6.5 \%)$ & 2011 & [131] \\
\hline $\mathrm{ZnO} / \mathrm{RGO}$ & 60 & $180(\sim 6 \%)$ & 2015 & [132] \\
\hline $\mathrm{ZnO} / \mathrm{GNS}$ & 291 & $1000(32.5 \%)$ & 2015 & [190] \\
\hline
\end{tabular}
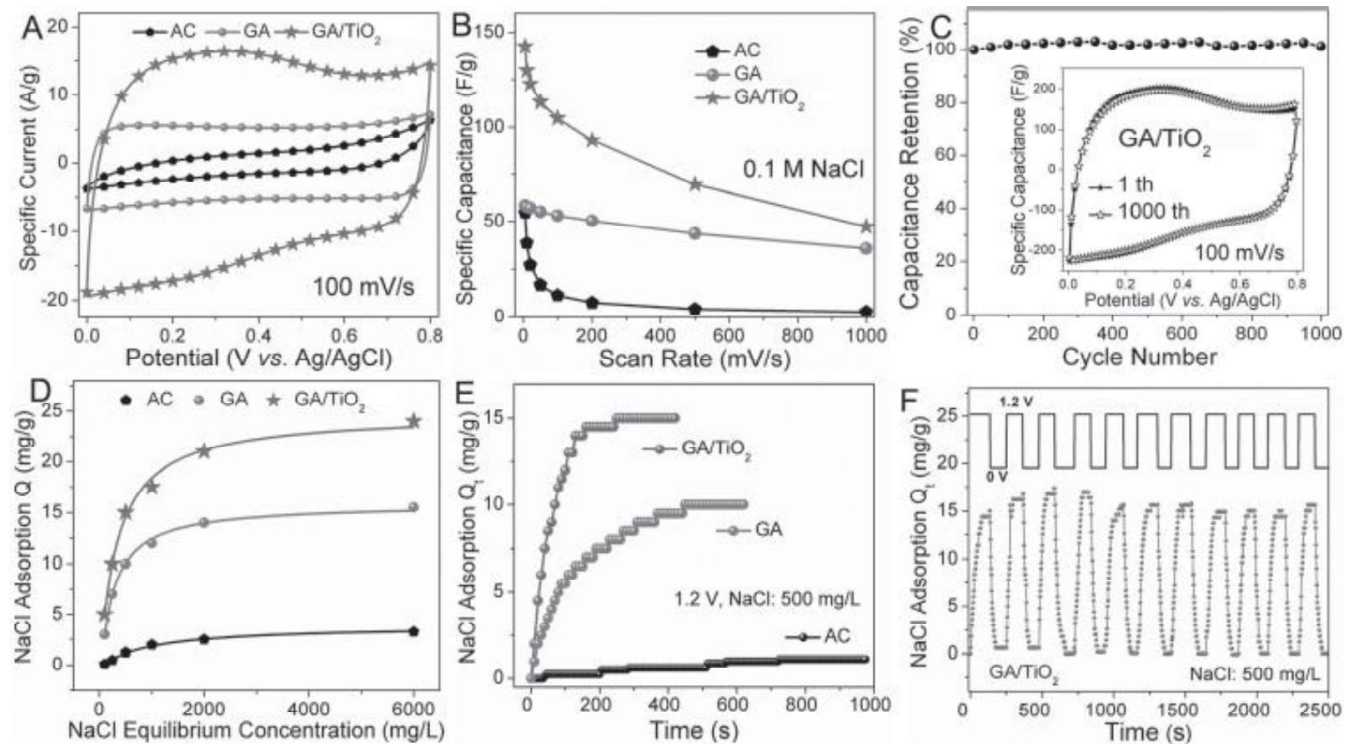

Fig 15. (A) CV curves of $\mathrm{AC}, \mathrm{GA}, \mathrm{GA} / \mathrm{TiO}_{2}$ in $0.1 \mathrm{M} \mathrm{NaCl}$ solution at a scan rate of $100 \mathrm{mV}$ $\mathrm{s}^{-1}$; (B) The specific capacitance of $\mathrm{AC}, \mathrm{GA}, \mathrm{GA} / \mathrm{TiO}_{2}$ at different scan rates; (C) Cycling performance of $\mathrm{GA} / \mathrm{TiO}_{2}$ at a scan rate of $100 \mathrm{mV} \mathrm{s}^{-1}$, inset presents the $\mathrm{CV}$ curves before 
and after the 1000 cycles. (D) Desalination capacity of AC, GA and GA/ $\mathrm{TiO}_{2}$ with different equilibrium concentrations and the corresponding Langmuir isotherm fitted curves; (E) Desalination efficiency of $\mathrm{AC}, \mathrm{GA}$ and $\mathrm{GA} / \mathrm{TiO}_{2}$ in $500 \mathrm{mg} / \mathrm{L} \mathrm{NaCl}$ solution at different times; (F) Electrosorption and regeneration cycles of $\mathrm{GA} / \mathrm{TiO}_{2}$ in $500 \mathrm{mg} / \mathrm{L} \mathrm{NaCl} .[134]$ (Wiley Interscience)

\subsection{Solar cells}

Relative to the traditional battery research on graphene-based materials, the use of such materials in solar-related green energy was also a popular research topic in recent years, including solar cell (mainly DSSC) [22, 140, 186] and photocurrent generation via hydrogen evolution by photocatalytic water splitting reaction [139]. Due to the low cost and high conversion efficiency $(\sim 12 \%)$ achieved by DSSC, research into such area was very popular since the 1990s [22]. Up to date, research into conventional graphene-based DSSC materials involves the use of $\mathrm{MO}_{\mathrm{x}}$-loaded 2D or 3D graphene support [22, 24, 138, 140, 197-199], or metal-loaded graphene $[78,186,226]$. It should be noted that all the above works were carried out at a pressure $50 \%$ higher than the atmospheric condition. Most of the device scale graphene-based photoanode driven DSSCs were similar to each other [22, 199]; this was illustrated in Figure 16 assembled by Siwach's group with a corresponding operation scheme [199]. Some research groups used 2D graphene-based materials as electrode for $\mathrm{MO}_{\mathrm{x}}$-loaded graphene-based DSSC [24, 138, 140, 197] or the blocking layer of the photoanode [198], while some applied the 3D GF [22]. The performance from different research works showed that the 2D graphene-driven DSSC exhibited strong cell performance, as reflected in the short circuit current $\left(\mathrm{J}_{\mathrm{sc}}\right)$ range of 11.6 to $15.90 \mathrm{~mA} \mathrm{~cm}{ }^{-2}$, open-circuit voltage $(\mathrm{OCV})$ range of 0.72 to $0.78 \mathrm{~V}$, and an efficiency range of 3.2 to $8.2 \%$ when $\mathrm{MO}_{\mathrm{x}} / \mathrm{rGO}$ with optimum composition of GO and photosensitive metal oxides were used [138, 140, 197-199]. Figure 17 illustrated a 
$\mathrm{J}-\mathrm{V}$ curve example where $\mathrm{ZnO}$ graphene (ZGR) was used with different graphene loadings as the photoanode of the DSSC assembled by Siwach's group [199]. Interestingly, in the case of the $\mathrm{ZnO}$-loaded 2D graphene-based DSSC electrode, sometimes it did not show any activity [24], while some of the cases showed strong activity [199]. This may be due to the difference in the nature of $\mathrm{ZnO} /$ graphenes synthesized by CVD and the sol-gel method, which caused a change of the chemical structure of the resulting $\mathrm{ZnO}$ /graphene. The performance of $\mathrm{MO}_{\mathrm{x}^{-}}$ loaded graphene-driven DSSC was associated with the relative $\mathrm{MO}_{\mathrm{x}}$ loading in $\mathrm{MO}_{\mathrm{x}}$-loaded graphene photoanode. Wei's group and co-workers observed that the performance of graphene- $\mathrm{TiO}_{2}(\mathrm{GT}-\mathrm{x}, \mathrm{x}=0-2.5 \mathrm{~mL} \mathrm{rGO})$ composite driven DSSC was enhanced when the amount of rGO increased from 0 to $1.0 \mathrm{~mL}$, and then depreciated when $\mathrm{x}>1.0 \mathrm{~mL}$ in the raw mixture for GT synthesis [197]. The origin of DSSC performance depreciation was the formation of a recombination center by excessive $\mathrm{rGO}$ surrounding the surface of $\mathrm{TiO}_{2}$ nanoparticles (NPs) in the GT DSSC, which results in longer charge migration route or electron directly coming into contact with electrolyte for dark current formation [197]. As a result, the optimum amount of graphene used for DSSC photoanode preparation should be fully considered. Some research groups like Tang's team [22] used 3D structured graphene synthesized from CVD, followed by chemical modification of GF with P25 and fabricated on a conductive glass for the photoanode preparation. The performance of the DSSC assembled by this 3DGN-P25 photoanode showed stronger output and efficiency than that driven by pure $\mathrm{P} 25$, achieving $15.4 \mathrm{~mA} \mathrm{~cm}{ }^{-2}, 673 \mathrm{mV}$, and $6.58 \%$ for $\mathrm{J}_{\mathrm{sc}}, \mathrm{OCV}$, and efficiency, respectively [22]. 
(a)

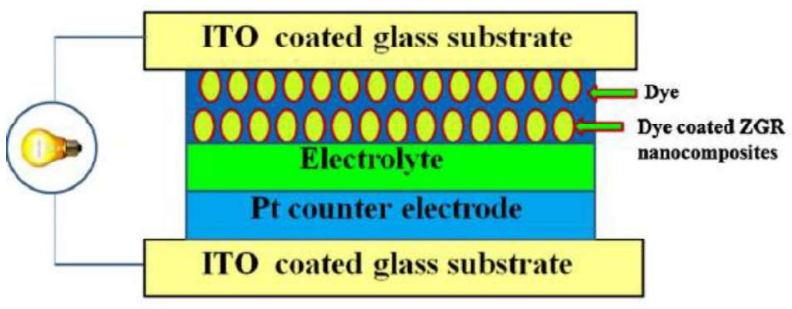

Fig 16. (a) Schematics of ZGR based DSSC

(b) Typical energy levels of various components of ZGR based DSSC employing an iodide/triiodide based redox electrolyte and N749 as a sensitizer.[199] (Springer)

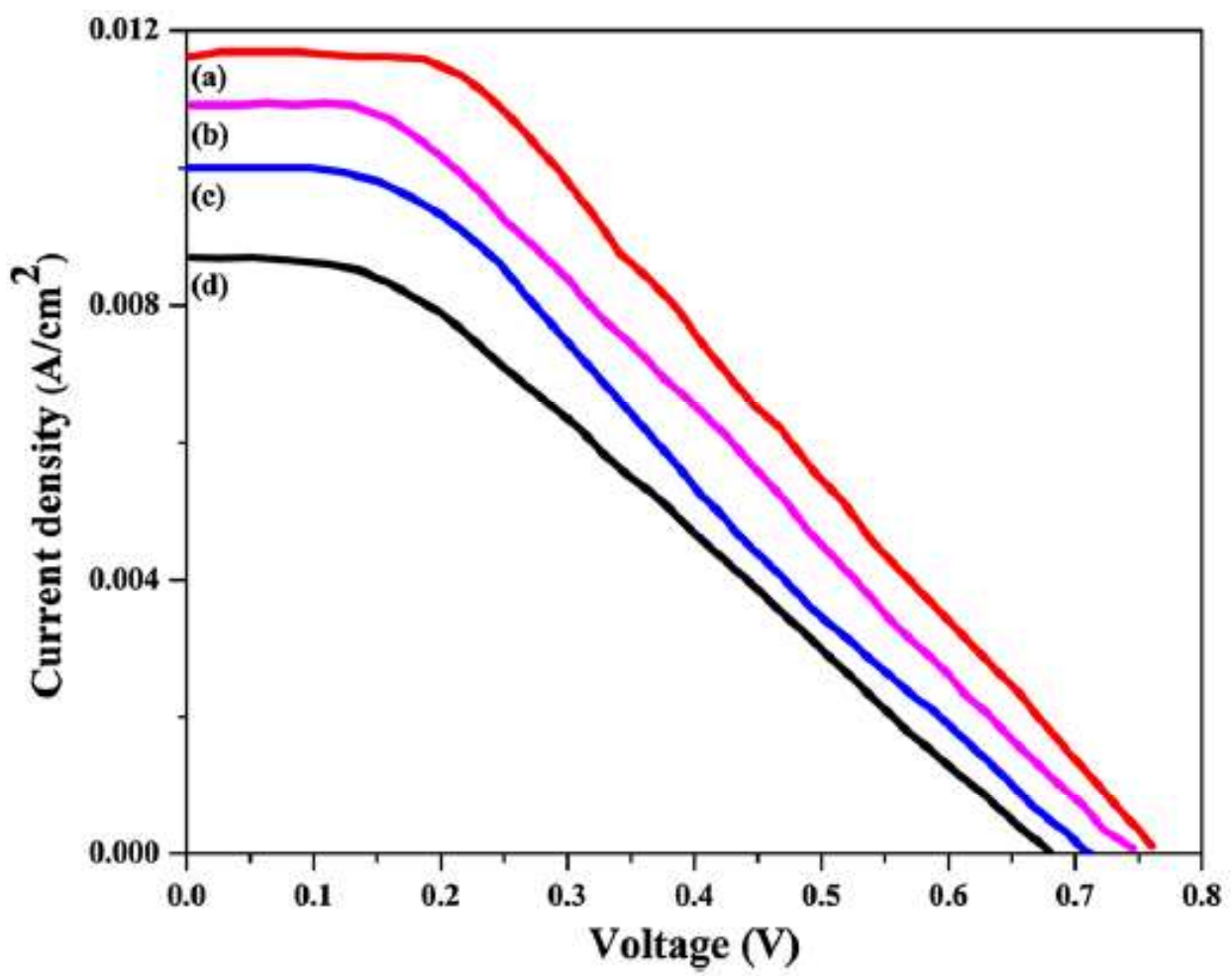

Fig 17. J-V curves of DSSCs based on (a) ZGR $0.25(b)$ ZGR 0.5 (c) ZGR 1 and (d) ZGR 0 nanocomposite films.[199] (Springer)

For the case of metal-loaded graphene, one of the examples was graphene-Ag NPs supported on $\mathrm{TiO}_{2}\left(\mathrm{TiO}_{2} /\right.$ Graphene-Ag NPs) fabricated by Huang's group [226], which 
exhibited strong electrical properties with a $\mathrm{J}_{\mathrm{sc}}$ recorded value of $14.3 \mathrm{~mA} \mathrm{~cm}{ }^{-2}$, OCV of 0.71 $\mathrm{V}$, and conversion efficiency of $6 \%$. Another example involves the use of Pt/rGO as counterelectrode, which showed enhanced activity compared to that of the counter-electrodes composed of Pt and rGO. This is reflected in the high output values of $\mathrm{J}_{\mathrm{sc}}$ of $11.6 \mathrm{~mA} \mathrm{~cm}{ }^{-2}$, OCV of $0.78 \mathrm{~V}$, and efficiency of $3.9 \%$ for the Pt/rGO-driven DSSC studied by Chen's group [186]. Recently, Yu's team even showed that metal-loaded 3D GA-like products can also act as both the MOR electrocatalysts and strong counter-electrode for DSSCs [78]. The performance recording the Pt/HGF-driven DSSC assembled from this electrode showed that it achieved a $5.6 \%$ conversion efficiency with $\mathrm{J}_{\mathrm{sc}}$ and $\mathrm{OCV}$ of $12.3 \mathrm{~mA} \mathrm{~cm} \mathrm{~cm}^{-2}$ and $0.68 \mathrm{~V}$, respectively. These results were comparable to the performance of $\mathrm{TiO}_{2}$-driven DSSC [78, 226]. Based on the summarized results shown in Table 8 , both $\mathrm{MO}_{\mathrm{x}}$-loaded graphene and metal-loaded graphene have strong potential for being solar cell electrode materials.

Table 8. List of best performance of graphene-based DSSC.

\begin{tabular}{|c|c|c|c|c|c|}
\hline Graphene photoanode & $\mathrm{J}_{\mathrm{sc}}\left(\mathrm{mA} \mathrm{cm} \mathrm{cm}^{-2}\right)$ & $\mathrm{OCV}(\mathrm{V})$ & Efficiency (\%) & Year & Ref \\
\hline 3DGN (1 wt $\%)-P 25$ & 15.4 & 0.67 & 6.6 & 2013 & {$[22]$} \\
\hline $0.75 \mathrm{wt} \%$ RGO-P25 & 12.2 & 0.67 & 5.5 & 2013 & [140] \\
\hline $\mathrm{Pt} / \mathrm{rGO}$ & 11.6 & 0.78 & 3.9 & 2013 & [186] \\
\hline Pt-HGF & 12.3 & 0.68 & 5.6 & 2017 & [78] \\
\hline GB-1.0 & 15.3 & 0.74 & 7.5 & 2017 & [198] \\
\hline ZGR 0.25 & 11.6 & 0.76 & 3.2 & 2017 & [199] \\
\hline $\mathrm{S}-\mathrm{Co}_{3} \mathrm{O}_{4} / \mathrm{rGO}$ & 15.9 & 0.76 & 8.2 & 2018 & [138] \\
\hline $\begin{array}{l}\text { Graphene } \\
\text { (GT-1.0) }\end{array}$ & 15.0 & 0.72 & 7.1 & 2018 & [197] \\
\hline $\mathrm{TiO}_{2} /$ Graphene-Ag NPs & 14.3 & 0.71 & 6.0 & 2018 & [226] \\
\hline
\end{tabular}

\subsection{Solar-driven water splitting reactions}

Metal oxide-loaded graphene-based materials also played an important role in the watersplitting reaction with improved performance of the photocurrent production when compared to the corresponding pure metal oxides $[139,200]$. From the investigation carried out by Ghorbani and Gao's group [139, 200], the photocurrent generated throughout the watersplitting reaction catalyzed by $\mathrm{ZnO} /$ graphene porous structure $\left(\mathrm{ZnO} / \mathrm{GO}_{(1 \mathrm{wt} \%)}\right)$ and (GO- 
$\left.\mathrm{TiO}_{2}-3\right)$ was $1.0 \mathrm{~mA} \mathrm{~cm}$ and $32 \mathrm{~mA} \mathrm{~cm}^{-2}$, respectively. These values were around approximately 12 times that of pure $\mathrm{ZnO}\left(0.09 \mathrm{~mA} \mathrm{~cm}^{-2}\right)$ and 3 times that of $\mathrm{TiO}_{2}(10 \mathrm{~mA}$ $\left.\mathrm{cm}^{-2}\right)$, respectively $[139,200]$. The hydrogen evolution rate recorded by $\mathrm{GO}-\mathrm{TiO}_{2}-3(305.6$ mmol h$\left.{ }^{-1}\right)$ was also much larger than that of $\mathrm{TiO}_{2}\left(23.5 \mathrm{mmol} \mathrm{h}^{-1}\right)$ [139]. Both groups suggested the improved charge separation within the $\mathrm{MO}_{\mathrm{x}}$ /graphene products upon irradiation by the light source $[139,200]$, which is reflected from the examples of the charge transfer scheme as shown in Figures 18-19 [139, 200]. These materials showed the potential of graphene-based products as electrode materials for hydrogen water-splitting cells in the future.

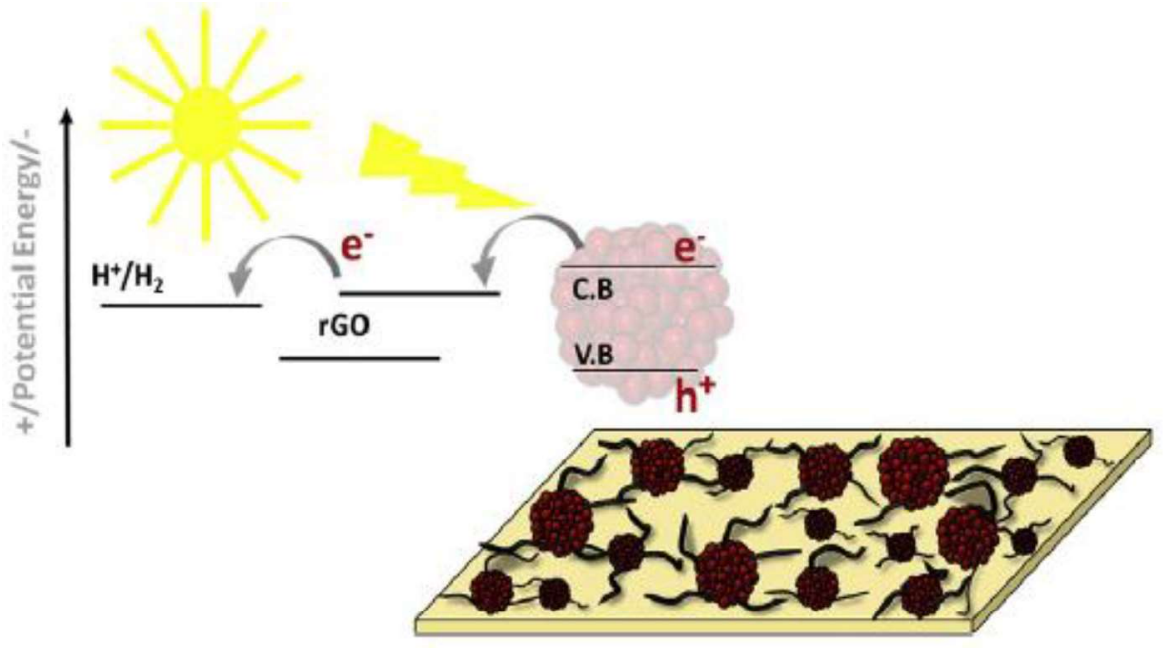

Fig 18. Electron transfer mechanism in $\mathrm{ZnO}$ nanoparticles and graphene/ $\mathrm{ZnO}$ nanocomposite on irradiation.[200] (Elsevier BV)

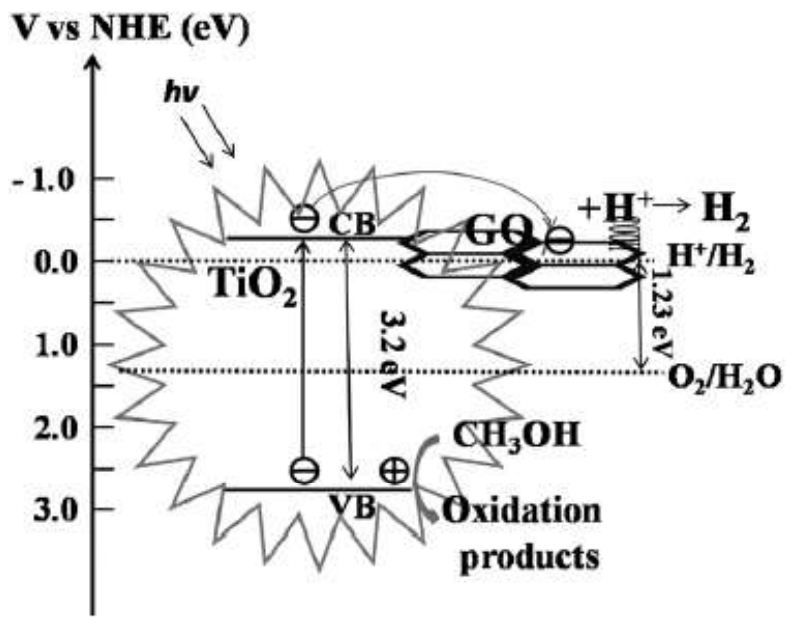


Fig 19. Mechanism of hydrogen production in the $\mathrm{GO}-\mathrm{TiO}_{2}$ composite system.[139] (Wiley Interscience)

\section{Recent developments and applications of new technology for graphene-based materials}

Even though graphene-based materials are attractive materials for green energy development as mentioned previously $[32,33,67-71,150,164,165,202-204]$ and shape customized GA can also be obtained by the use of a suitable shape reactor [64] or NF as template after a simple soaking with chemical reduction [32, 33, 67-71], several shortcomings still exist as a barrier to further development using traditional technology. For example, a complicated process is necessary to fabricate graphene-based materials or catalysts for electrode production, and the risk of original structure destruction during ultrasonication exists. More importantly, such a process requires the use of surfactant to stabilize the graphene catalyst on the support surface, especially 3D GA-based products. Using direct growth GA-based materials on support like NF may also have potential problems for catalytic activity exhibited from the support itself. It is difficult to synthesize large-sized customized GA-derivative electrodes by the traditional hydrothermal or mild chemical reduction method due to the requirement of a specific reactor with the desirable size, resulting in high production cost. The contraction in product size due to the surface tension of the reaction mixture exhibited in the GA products throughout the self-assembly process also exists. Even though the GF synthesis can correct this shortcoming via CVD with the use of $\mathrm{NF}$ or $\mathrm{Cu}$ foil, the removal of CVD supports ( $\mathrm{Cu}$ foil and $\mathrm{NF})$ was also complicated and involved the use of a corrosive or toxic etchant, which makes the synthesis not user-friendly. All these shortcomings may lead to actual activity not being reflected in the operation and 
limit the value of binder-free GA-based green energy materials for large-scale production and real device-level application.

To overcome this barrier, the importation of novel technology like 3D printing has recently become a new direction. It is because 3D printing technology can eliminate the weakness of GH size contraction due to surface tension throughout the traditional GA synthesis $[64,148]$. It can also achieve the size and shape scalable production by using computer designed (CAD) shapes instead of using specific size of shape reactor $[64,148]$. It is extremely suitable for scalable free-standing bulk GA electrode synthesis. The most commonly used 3D printing technology for graphene synthesis includes three methods: fused deposition modeling (FDM) [10, 11, 227-234], stereolithography (SLA) [235-241], and direct ink writing (DIW) $[12,13,242-248]$. The difference in the synthesized GA process between 3D printing and traditional chemical reaction methods is illustrated in Figure 20 [148, 249], where Figures 20a-c belongs to SLA method, Figure 20d belongs to FDM, Figure 20e is DIW, and Figures 20f-g is traditional hydrothermal reaction for the 3D GA synthesis with NSGAs as example. The major difference is that traditional methods required the use of a specific shape container to achieve scalable GA [64], but the size contraction throughout selfassembly is unavoidable (Figures 1a, 20f and 20g) [64, 148]. Such phenomena do not take place in the 3D printing process [250]. The only similarity is preparation of GO-based dispersion is the first step of both techniques. 


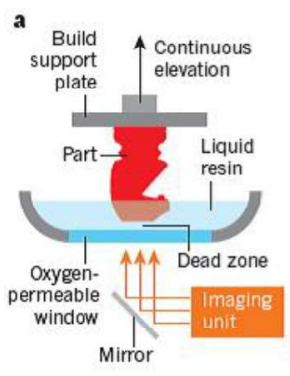

(f)

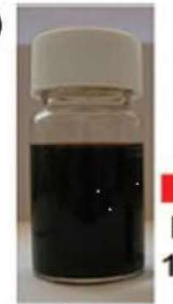

Graphene oxide b

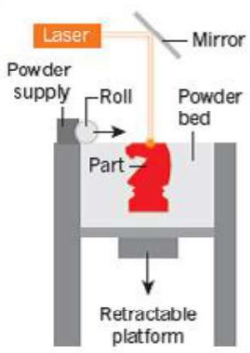

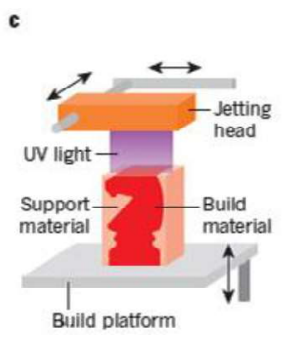

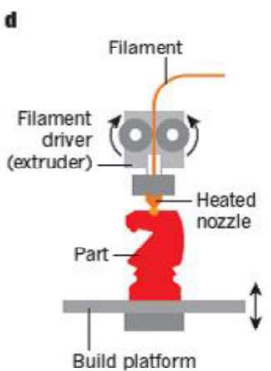

-

(g)

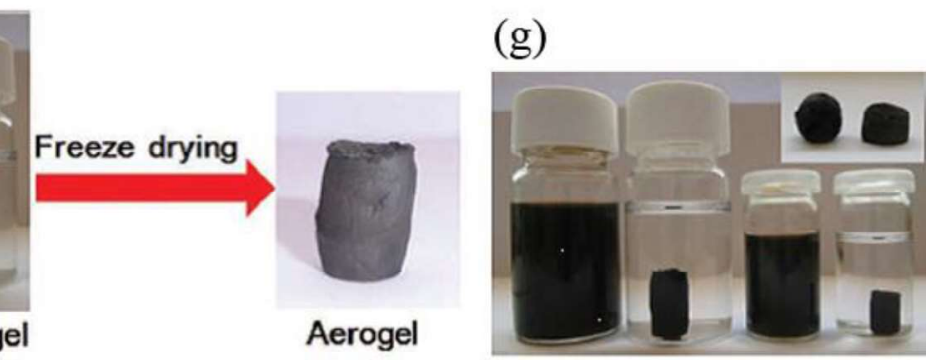

Fig 20. Common light- and ink-based 3D printing methods. a, The light-based 3D printing method known as continuous liquid interface production. (Diagram adapted from [250]) b, Light-based selective laser sintering of powders. c, Light- and ink-based photocurable inkjet printing of photopolymerizable resins. $\mathrm{d}$, Ink-based fused deposition modelling of thermoplastic filaments. e, Direct ink writing using viscoelastic inks [249] (Nature group). f, Fabrication illustration of $\mathrm{BN}-\mathrm{GAs}$ hydrothermal process and freeze-drying process. $\mathrm{g}$, Digital images of two pairs of GO solutions with different volumes in the vials and N/B codoped graphene hydrogels before and after hydrothermal self-assembly. Inset: digital images of the BN-GAs obtained after freeze-drying [148] (Wiley Interscience).

FDM is a printing technique involving the use of an ink-like plastic filament. The 3D structure is formed by melting the filaments into a paste-like thread at the nozzle of the printing head, followed by solidification on the printing platform to obtain the product $[10,11$, 227-229, 232-234]. SLA involves the use of photolithographic resin with a hardening of the resin and laser cutting of the resin slurry throughout the synthesis [235-241]. DIW, an extrusion-based direct printing technique which is relatively simple since the liquid or pastelike graphene-based ink can be used directly in the printing process by a printer $[12,13,234$, 
242-248]. The common characteristics of these printed products were size and shape scalable by the computer program like CAD drawing and Solidworks [10-13, 227-232, 234-248]. An example is shown in Figure 21 as demonstrated by Manapat's group via the SLA technique [239]. These products exhibited characteristics comparable to the traditional GA, such as mechanical strength $[227,228,234-236,238-240]$ and electrical conductivity $[10,228,230$, 231, 236, 241]. For example, Manapat's group found that the mechanical strength of the SLA-printed GO-loaded products was enhanced when the amount of GO in the polymer resin increased, and the 3D-printed GO composites exhibited stronger mechanical strength than the casted GO products as revealed by the tensile strength variations shown in Figures 21-22 [239].

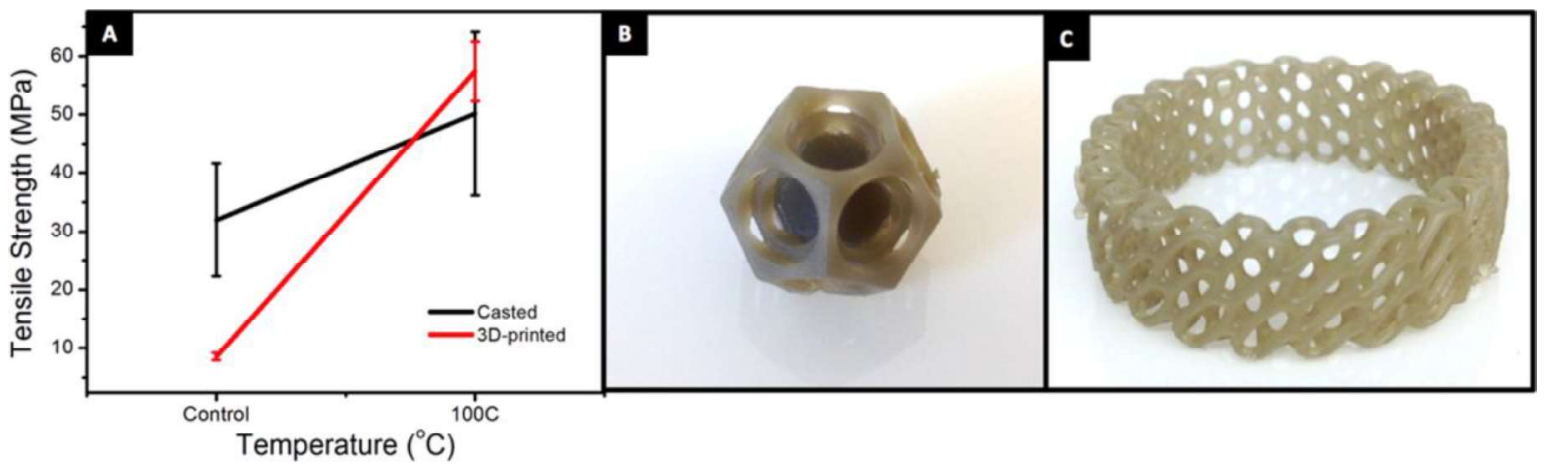

Fig 21. (A) Tensile strength comparison of cast and 3D-printed parts. SLA-printed complexshaped GO nanocomposites: (B) nested dodecahedron and (C) diagrid ring.[239] (ACS)

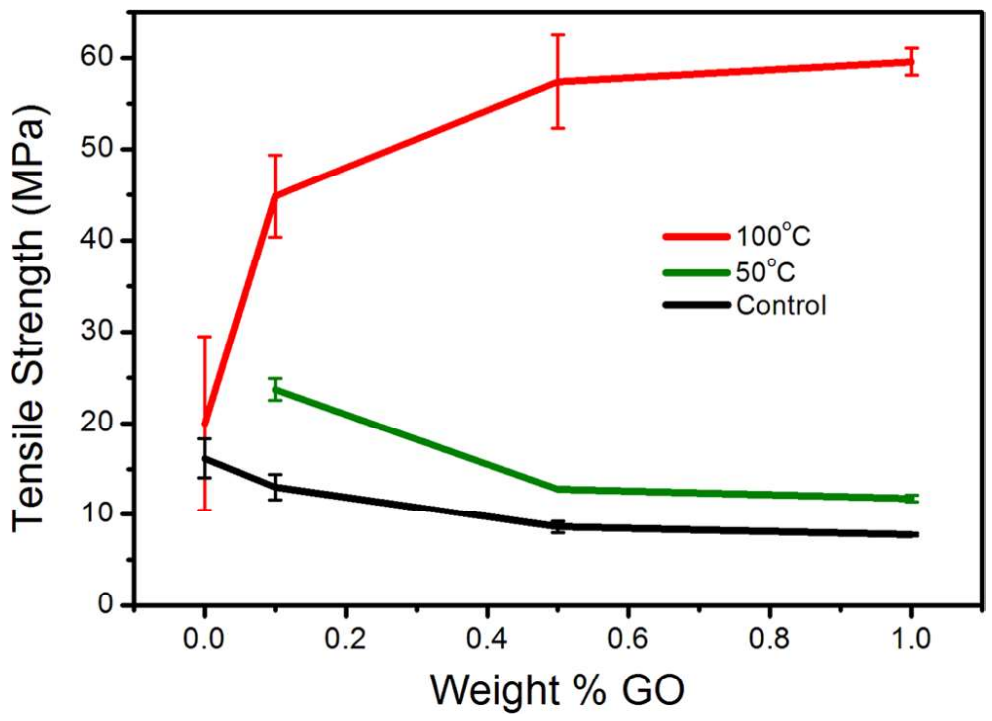


Fig 22. Tensile strength as a function of GO loading.[239] (ACS)

Similar to SLA mentioned previously, FDM and DIW GA products also showed the size- and shape-customized nature, as reflected in the digital images and SEM images illustrated in Figure 23 from Foo's team (FDM) [232] and Figure 24 obtained from Zhu's group (DIW GAs) [243] as representatives. So far, pure GA [12, 232, 242-246], metal (M) [12, 232, 246], metal oxides [247, 248], and metal sulfide (MS) [12], or organic moleculeloaded GAs [13] were demonstrated in both FDM and DIW method, which showed size-, porosity-, and shape-customized features. The loading uniformity of foreign materials (metal/metal sulfide) in the 3D-printed GA array was also high [12, 232, 246, 247].

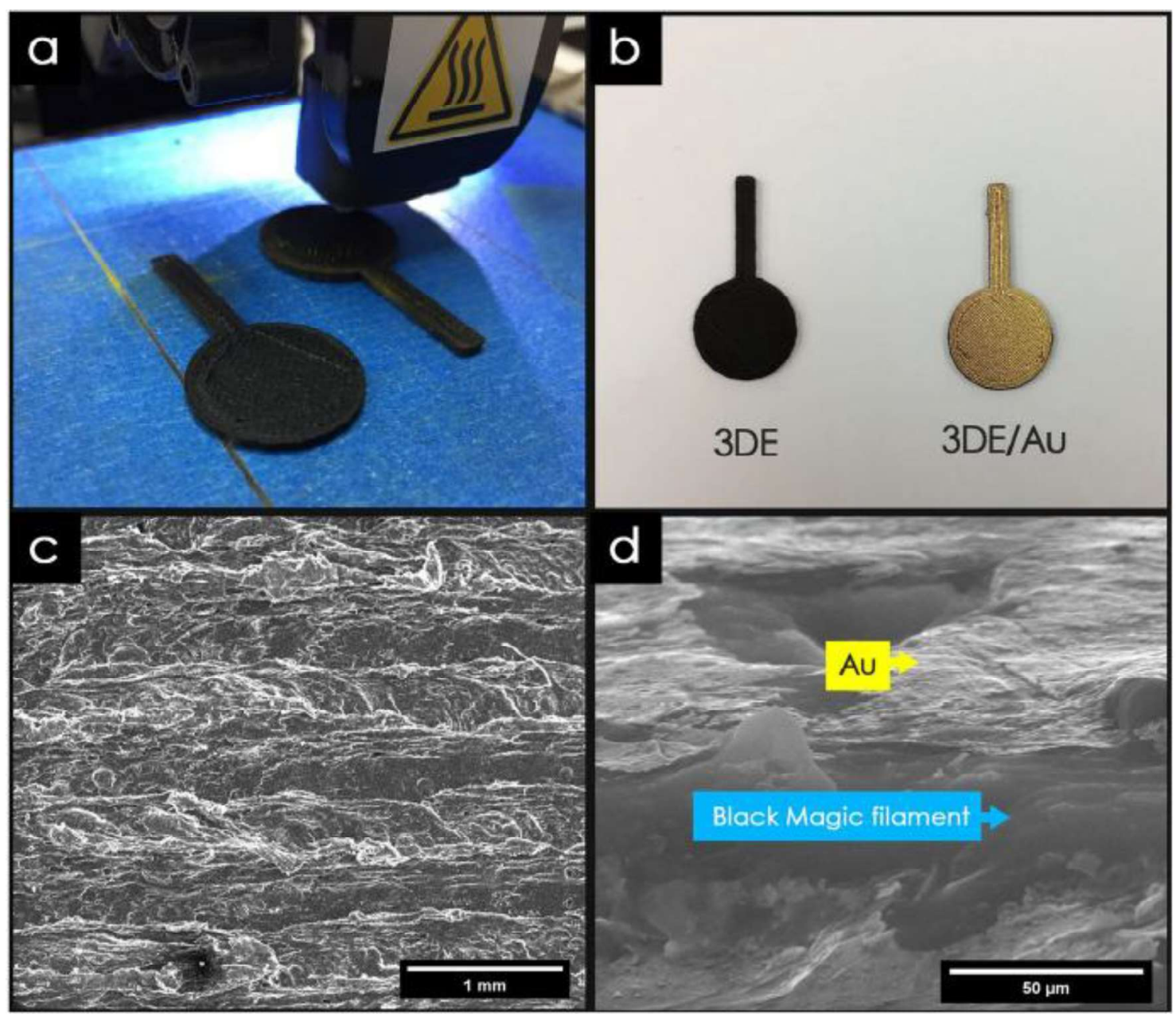

Fig 23. Physiochemical characterization. (a) Optical image of the 3D printing process, (b) 3D 
printed electrode used throughout the study. (c) FESEM image of 3DE/Au electrode, and (d) corresponding magnified cross-sectional area. [232] (Nature group)

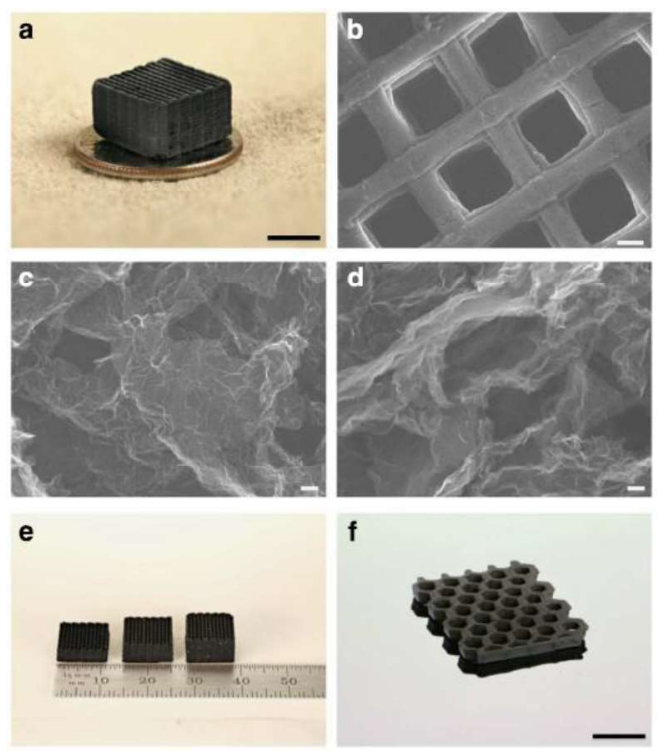

Fig 24. Morphology and structure of graphene aerogels. (a) Optical image of a 3D printed graphene aerogel microlattice. SEM images of (b) a 3D printed graphene aerogel microlattice, (c) graphene aerogel without R-F after etching and (d) graphene aerogel with $4 \mathrm{wt} \% \mathrm{R}-\mathrm{F}$ after etching. Optical image of (e) 3D printed graphene aerogel microlattices with varying thickness and (f) a 3D printed graphene aerogel honeycomb. Scale bars, 5mm (a), 200mm (b), $100 \mathrm{~nm}(\mathrm{c}, \mathrm{d}), 1 \mathrm{~cm}(\mathrm{f}) \cdot[243]$ (Nature group)

To the best of our knowledge, only a few studies focused on the size- and shapecustomized 3D-printed 2.5D and 3D pure GA blocks via the binder-free aqueous GO solution by ice-supported DIW method as illustrated in Figure 25, which showed mechanical and electrical properties comparable to those synthesized by traditional methods [244]. This method has the advantage of a totally additive-free nature; its mechanical and electrical properties were hence wholly contributed from the pure graphene itself, as reflected by the real-time mechanical results reported by Zhang et al. (Figure 26) [244] Recently, another demonstration carried out by Ma and his coworker showed the bulk GA can also be printed 
out under room temperature (ice mold-free printing) via the use of a large GO [245]. Illustrations in Figures 27-28 showed the flow of ambient environment GA printing, strong electrical and mechanical characteristics, and size- and shape-customized properties of 3Dprinted GA products [245]. Such properties are comparable to the ice-supported printed GA (Figures 25-26). Both ice-supported printing [244] and ambient printing [245] technique showed the advantage of shape and array customized properties. The printed GA exhibited strong mechanical strength (Young's Modulus=137 kPa (80\% strain)-174.6 kPa (50\% strain)) and electrical conductivity $\left(15.4-41.1 \mathrm{~S} \mathrm{~m}^{-1}\right)[244,245]$.

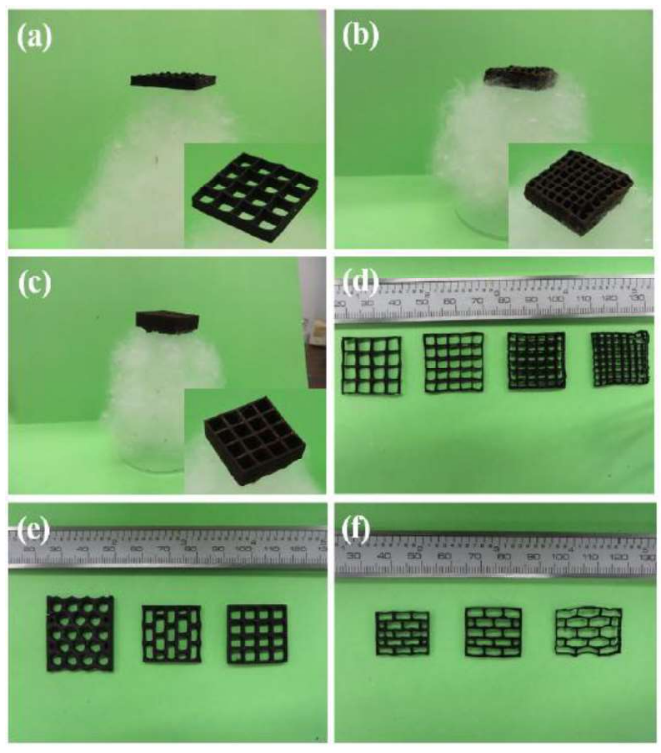

Fig 25. 3D printed GO aerogels. (a)-(c) Printed GO aerogels on catkin, and (d)-(f) designs of printed GO aerogels.[244] (Wiley Interscience)

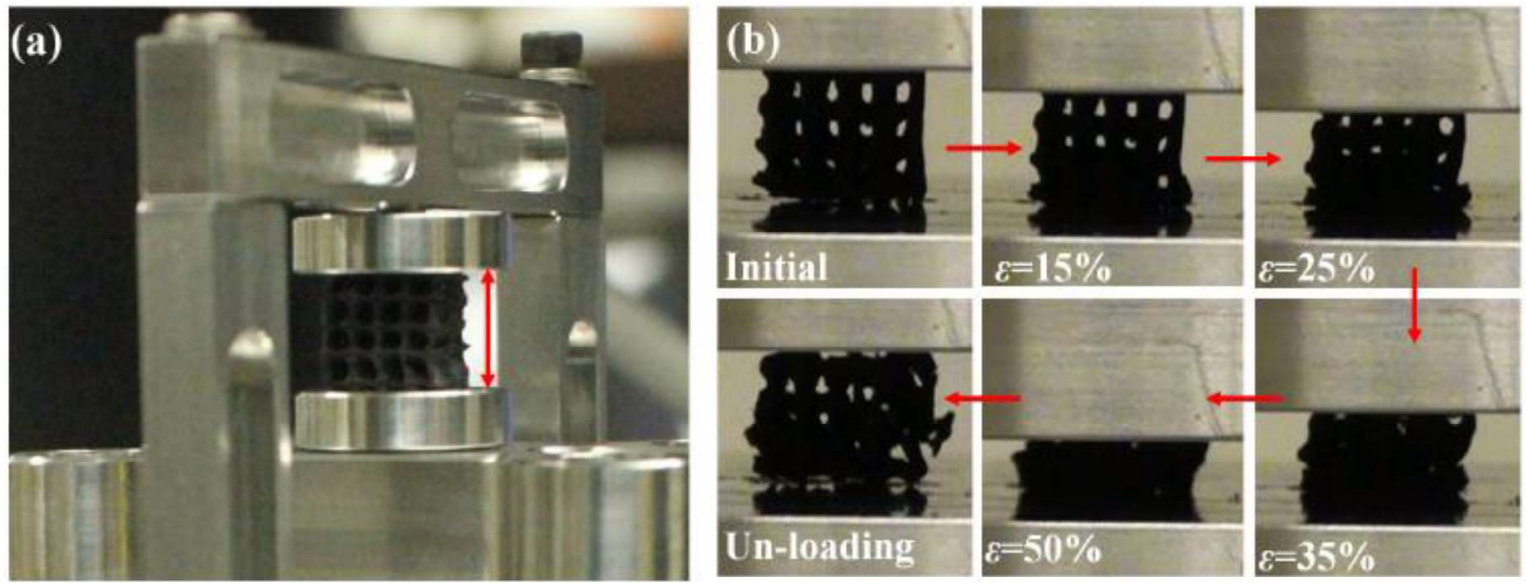


Fig 26. (a) Experimental setup for compression by DMA. (b) Loading and unloading process during compression test (50\% strain).[244] (Wiley Interscience)
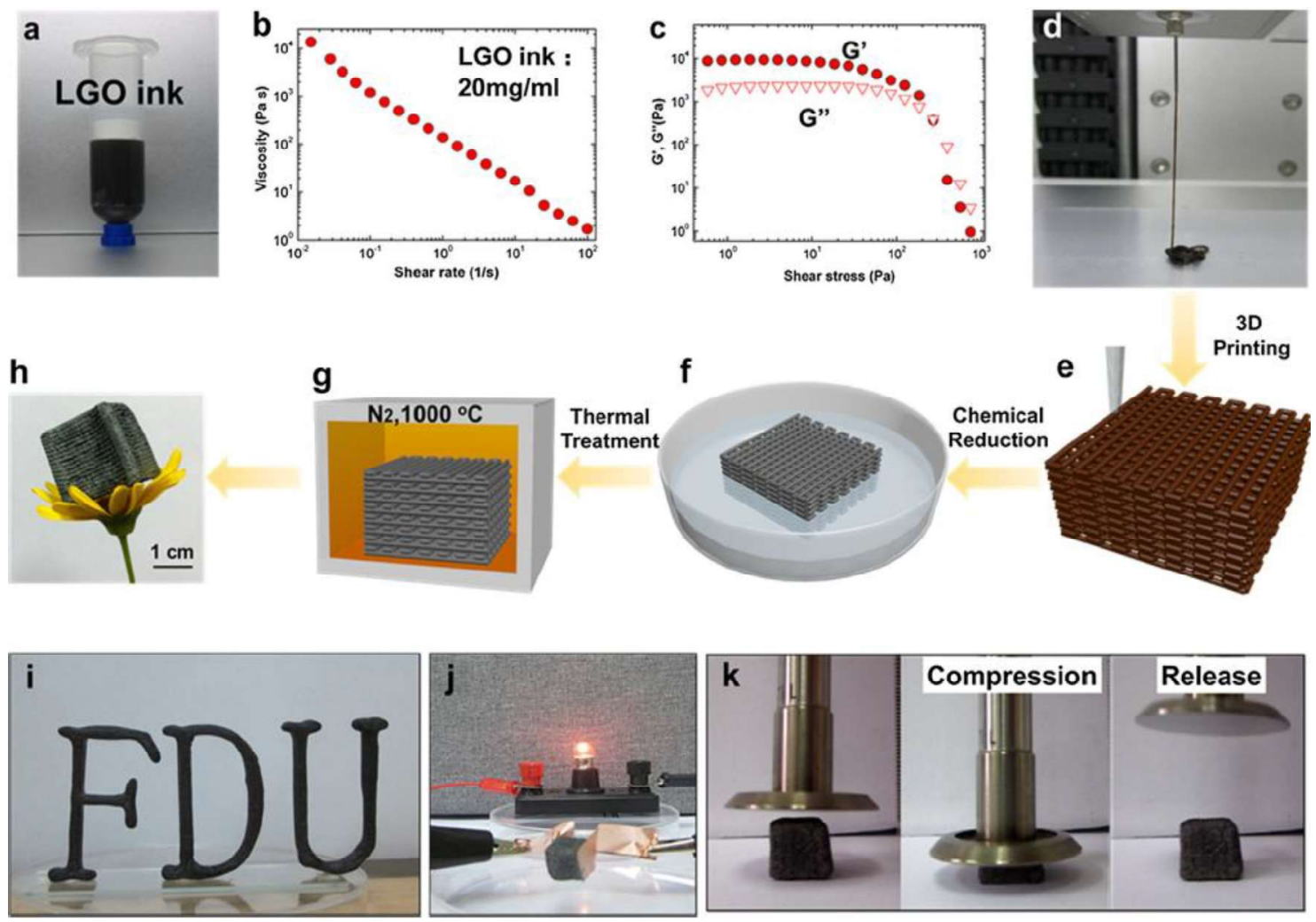

Fig 27. LGO ink properties and schematic diagram of the fabrication process (a) Digital image of the LGO ink with a concentration of $20 \mathrm{mg} / \mathrm{mL}$ in a $30 \mathrm{~cm}^{3}$ barrel, (b) Viscosity as a function of the shear rate for LGO ink, (c) G0 and G00 as a function of the shear stress for LGO ink, (d) the LGO ink extrusion through micro needle $(400 \mu \mathrm{m})$, (e) the model of printed 3D LGO monolith, (f) chemical reduction of LGO monolith by HI, (g) thermal annealing of monolith at $1000{ }^{\circ} \mathrm{C}$ in nitrogen, (h) the optical image of the final low-density $3 \mathrm{D}$ graphene monolith, (i) the printed 3D letter FDU, (j) the electrical property of T-LGO monolith, (k) the compressive and resilient property of T-LGO monolith.[245] (Elsevier B. V.)

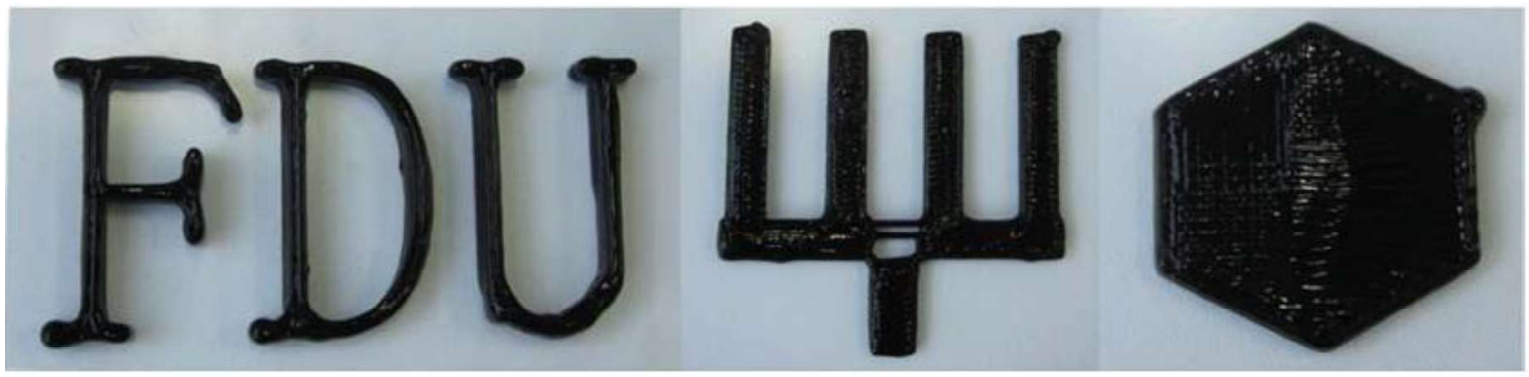


Fig 28. The photograph of the printed 3D letter FDU, fork type and hexagon.[245] (Elsevier B. V.)

FDM and DIW 3D printed graphene products played important role in green energy research, include hydrogen evolution reaction [10], LIBs [10], supercapacitor [10-13, 232, $247,248]$, and photocurrent [232]. These products were pure graphene in nature [10-13, 232]; only a few of them were foreign materials loaded 3D graphene via FDM (gold loaded threedimensional Printed Electrode (3DE/Au)) [232] and DIW (3D printed graphene aerogel/ $\mathrm{MnO}_{2}\left(3 \mathrm{D} \mathrm{G} / \mathrm{MnO}_{2}, \mathrm{MOG}\right), \mathrm{NiO}$ or $\mathrm{MnO}_{2}$ loaded integrated graphene network (IGF) (NOG, MOG)) [247, 248] respectively. For example, Foster's group demonstrated that the FDM 3D-printed graphene/polylactic acid (graphene/PLA) exhibited strong electrochemical activity (Current density $=0.4 \mathrm{~A} \mathrm{~g}^{-1}$ in $\left.3 \mathrm{M} \mathrm{KOH}\right)$ and the capacitance $\left(4.79 \mathrm{~F} \mathrm{~g}^{-1}\right.$ in $\left.3 \mathrm{M} \mathrm{KOH}\right)$ from the coin battery assembled with the graphene/PLA electrode (Figures 29-30) [10]. Its strong hydrogen evolution reaction activity (Onset potential=-0.84 V (vs SCE)) was reflected from the strong current density output illustrated in Figure 31 by comparison to other graphite-based materials [10]. DIW GA based products also exhibited strong electrical conductivity $\left(71.5-120 \mathrm{~S} \mathrm{~m}^{-1}\right)[12,13,242]$, high specific capacitance $\left(4.79-239.7 \mathrm{~F} \mathrm{~g}^{-1}\right)$ and operation stability up to $1000-20000$ cycles of operation [12, 13, 247, 248], as reflected in the cyclic voltammetric results in the electrochemical performance study carried out by Tang's group (Figure 32) [12]. By comparing with those involving the GA based materials synthesized by traditional method as listed in Table 9, which showed that 3D printed GA products have comparable activity and are new stars as replacement materials of synthesized GA by traditional method in the green energy application.

Table 9. List of green energy research materials involving the use of 3D printed GA based materials against traditional GA based products.

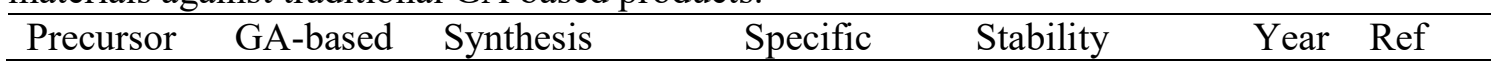




\begin{tabular}{|c|c|c|c|c|c|c|}
\hline Type & composite & method & $\begin{array}{l}\text { capacitance } \\
\left(\mathrm{F} \mathrm{g}^{-1}\right)\end{array}$ & (Depreciation) & & \\
\hline \multirow[t]{3}{*}{ None } & GA@NF & $\begin{array}{l}\text { Chemical } \\
\text { reduction }\end{array}$ & 366 & $\begin{array}{l}2000 \quad \text { cycles } \\
\left(15 \% \quad\left(2 \mathrm{~A} \quad \mathrm{~g}^{-}\right.\right. \\
\left.\left.{ }^{1}\right)\right)\end{array}$ & 2013 & {$[33]$} \\
\hline & $\begin{array}{l}\mathrm{RGO} / \mathrm{Ni} \\
\text { foam }\end{array}$ & Hydrothermal & 137 & $\begin{array}{l}10000 \text { cycles } \\
(\sim 0 \%)\end{array}$ & 2016 & {$[68]$} \\
\hline & $\begin{array}{l}\text { Graphene/ } \\
\text { PLA }\end{array}$ & $\begin{array}{l}3 \mathrm{D} \\
(\mathrm{FDM})\end{array}$ & 5 & $\begin{array}{l}120 \quad \text { cycles } \\
(\mathrm{N} / \mathrm{A})\end{array}$ & 2017 & {$[10]$} \\
\hline \multirow[t]{2}{*}{ MWCNT } & GCA@NF & $\begin{array}{l}\text { Chemical } \\
\text { reduction }\end{array}$ & 207 & $\begin{array}{l}2000 \text { cycles } \\
\left(20 \%\left(10 \mathrm{~A} \mathrm{~g}^{-}\right.\right. \\
\left.\left.{ }^{1}\right)\right)\end{array}$ & 2013 & {$[33]$} \\
\hline & $\begin{array}{l}\text { Graphene/ } \\
\text { MWCNT } \\
\text { MDHA }\end{array}$ & $\begin{array}{l}3 \mathrm{D} \\
(\mathrm{DIW})\end{array}$ & 27 & $\begin{array}{l}10000 \quad \text { cycles } \\
(10 \%)\end{array}$ & 2018 & {$[12]$} \\
\hline \multirow[t]{2}{*}{ PANI } & $\begin{array}{l}\text { PANI@3 } \\
\text { DGFs }\end{array}$ & $\begin{array}{l}\text { Hydrothermal, } \\
\text { electrochemical } \\
\text { deposition }\end{array}$ & 932 & $5000(29.8 \%)$ & 2015 & [162] \\
\hline & $\begin{array}{l}\mathrm{PANI}_{0.4} / \mathrm{R} \\
\mathrm{GO}\end{array}$ & $\begin{array}{l}3 \mathrm{D} \\
(\mathrm{DIW})\end{array}$ & 423 & $\begin{array}{l}1000 \quad \text { cycles } \\
(25 \%)\end{array}$ & 2018 & [13] \\
\hline $\mathrm{M}$ & $3 \mathrm{DE} / \mathrm{Au}$ & $\begin{array}{l}\text { 3D } \\
(\mathrm{FDM})\end{array}$ & 98 & $\begin{array}{l}1000 \text { cycles } \\
(87.9 \%)\end{array}$ & 2018 & [232] \\
\hline \multirow[t]{4}{*}{$\mathrm{MO}_{\mathrm{x}}$} & $\begin{array}{l}\text { 3D- } \\
\text { NiGOM }\end{array}$ & $\begin{array}{l}\text { Sol-gel } \\
\text { reduction }\end{array}$ & 1186 & $1000(22 \%)$ & 2015 & [69] \\
\hline & $\begin{array}{l}3 \mathrm{D} \\
\mathrm{G} / \mathrm{MnO}_{2}\end{array}$ & $\begin{array}{l}3 \mathrm{D} \\
(\mathrm{DIW})\end{array}$ & 239 & $\begin{array}{l}20000 \text { cycles } \\
(7.1 \%(2 \mathrm{~mm}))\end{array}$ & 2019 & [247] \\
\hline & MOG & $\begin{array}{ll}3 \mathrm{D} & \text { printing } \\
\text { (DIW) } & \end{array}$ & $121\left(\mathrm{~F} \mathrm{~cm}^{-2}\right)$ & $\begin{array}{l}10000 \text { cycles } \\
(13.4 \%)\end{array}$ & 2019 & [248] \\
\hline & NOG & $\begin{array}{ll}3 \mathrm{D} & \text { printing } \\
(\mathrm{DIW}) & \end{array}$ & 400 & $\begin{array}{l}10000 \text { cycles } \\
(13.9 \%)\end{array}$ & 2019 & [248] \\
\hline
\end{tabular}

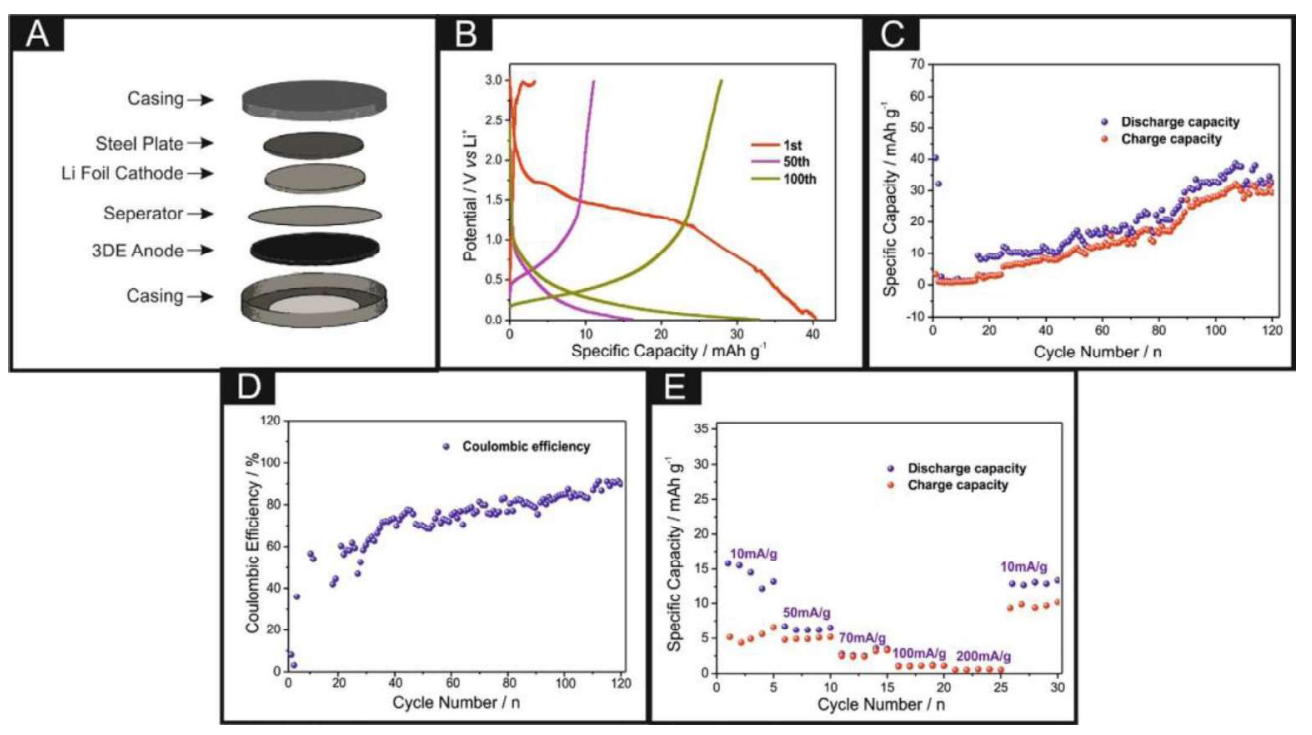


Fig 29. Schematic of the coin cell fabrication (A), charge-discharge profiles (B), cycling properties (C), coulombic efficiency (D) and rate capability of the 3D printed anode (E).[10] (Nature group)

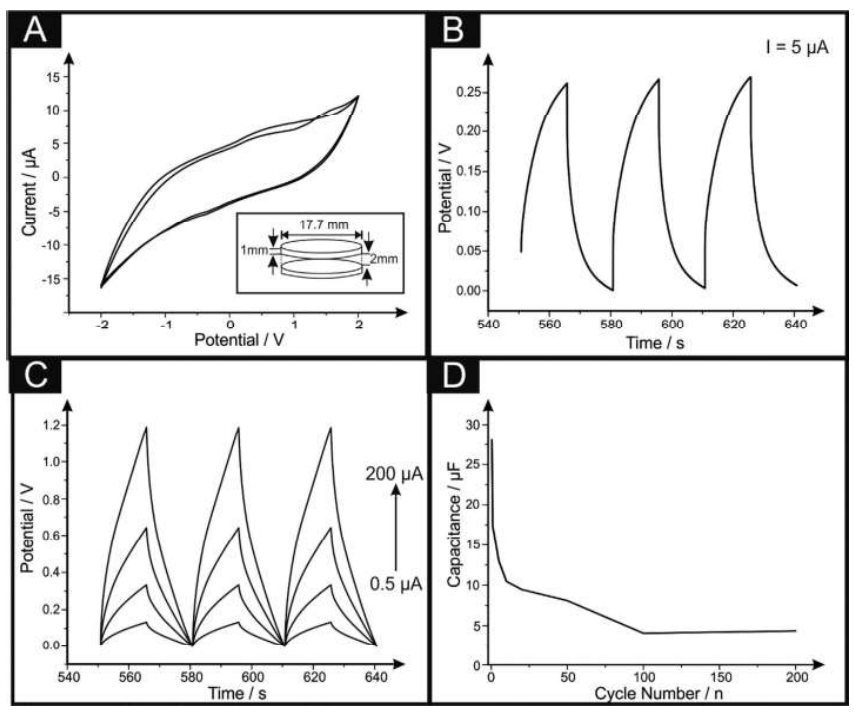

Fig 30. Cyclic voltammetry (A) of the 3D-SC consisting of a $2 \mathrm{~mm}$ layer of solid electrolyte of PVA and 1.0 $\mathrm{M} \mathrm{H}_{2} \mathrm{SO}_{4}$. Corresponding charge/discharge curves with (C) and without (B) the Kampouris' circuit in parallel are also presented. Scan Rate: $25 \mathrm{mV} \mathrm{s}^{-1}$. Inset to A is a schematic of the 3D-SC utilised throughout this study.[10] (Nature group)

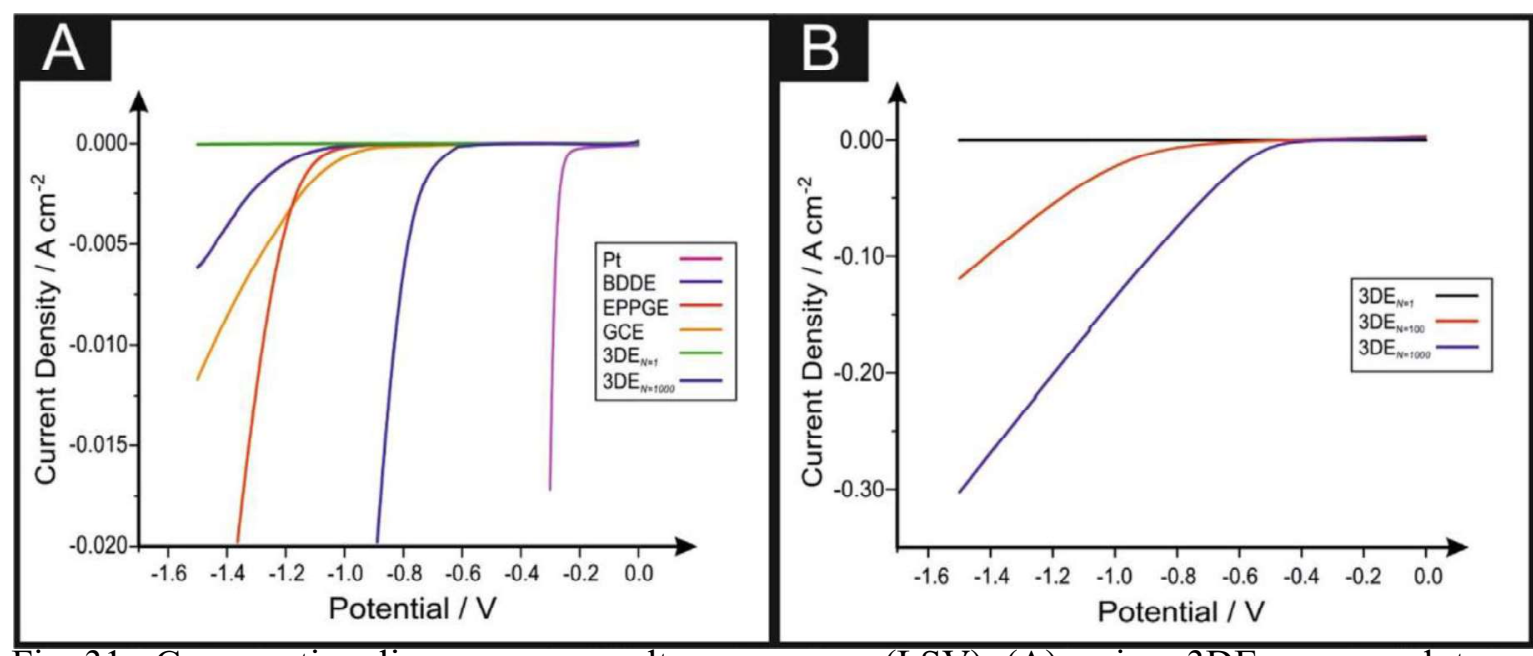

Fig 31. Comparative linear sweep voltammograms (LSV) (A) using 3DE compared to EPPGE, GCE, BDDE and platinum showing the onset of the HER. Stability studies of the 3DEs (B) using LSV for the initial, 10th, 100th and 1000th scans. Scan rate: $25 \mathrm{mV} \mathrm{s}^{-1}$ (vs. 
$\mathrm{SCE}$ ). Note: $3 \mathrm{DEN}=1$ is upon the initial scan and $3 \mathrm{DEN}=1000$ is upon the 1000 th scan.[10] (Nature group)
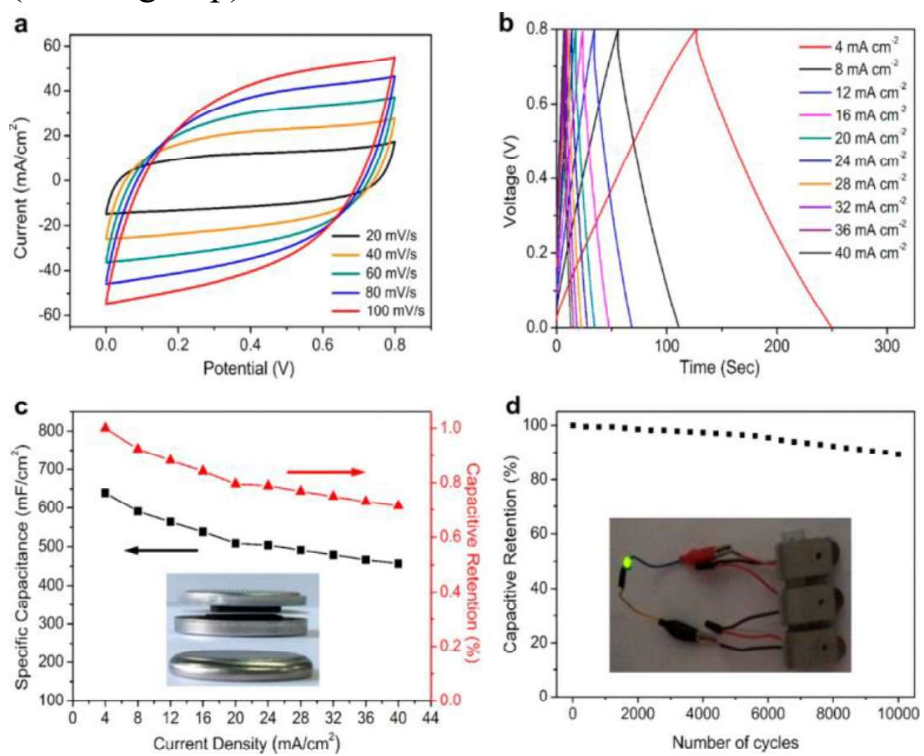

Fig 32. Electrochemical performance of the coin-type symmetric microsupercapacitors. (a) $\mathrm{CV}$ curves collected at scan rates of $20-100 \mathrm{mV} / \mathrm{s}$ in $6 \mathrm{M} \mathrm{KOH}$ electrolyte. (b) GCD profiles collected at current densities from 4 to $40 \mathrm{~mA} \mathrm{~cm}^{-2}$. (c) Areal capacitance and capacitive retention versus diff erent areal current densities $\left(4-40 \mathrm{~mA} \mathrm{~cm}^{-2}\right)$. The inset shows optical images of the device before and after assembling. (d) Cycling stability measurements. The inset shows an optical image of a green LED-lighted by three coins connected in series.[12] (ACS)

Even though the 3D-printed GA products showed strong activity in the green energy applications, including LIBs, and supercapacitor [10-13, 232, 247, 248], the materials used still suffer from the weakness associated with the printing techniques. Firstly, even though the use of 3D printing technology can produce high-resolution products in a low-cost way, SLA required long printing time (hourly) to achieve this target [250], and the solvent for mixing both GO and SLA resin is limited [235]. This makes SLA unsuitable for the GA based green energy electrode fabrication. Second and the most critically, synthesis of such 
ink required the use of surfactant (such as lactose, cellulose, polyvinyl alcohol (PVA), or polyvinyl butyral (PVB)) with prolonged synthesis time or direct use of the market-available filament ink $[10-13,231,232,242]$ to ensure the printable ink have proper rheological property. The metal-loaded FDM graphene 3D electrode even needs the spraying of gold and further fabrication of GO with the help of organic compounds [232]. This is especially obvious for FDM products $[10,11,231,232]$. The active catalytic sites would be blocked by the surfactant or resins, which may inhibit the contact between reactants and the loaded foreign materials in the $3 \mathrm{D}$ printed GA array $[12,232]$. Even though there is an example of binder-free foreign materials loaded 3D printed GA monolith (ruthenium loaded reduced 3D printed holey graphene oxide mesh ( $\mathrm{Ru} / \mathrm{r}-\mathrm{hGO}$ mesh), 3D G/MnO${ }_{2}, \mathrm{NOG}$ and MOG), it was synthesized by a complicated 2-step synthesis via soaking of the as-printed r-hGO mesh into the metal solution followed by further chemical reduction [246], or electrodeposition of $\mathrm{MnO}_{2}$ or NiO to the DIW 3D printed GA $[247,248]$. Such barriers need to be overcome for the GA-based materials via 3D printing technology in green energy research.

Based on the advantages and disadvantages of the above-mentioned 3D printing technology for the graphene-based green energy research as summarized in Table 10, use of DIW provides a solution of unleashing the potential of developing low-cost and binder-free freestanding foreign materials-loaded 3D-printed GA based catalysts for direct fuel cell, LIBs, supercapacitor, or solid oxide fuel cell electrodes in future.

Table 10. Advantages and disadvantages of the existing 3D printing methods for graphenebased green energy applications.

\begin{tabular}{lll}
\hline 3D printing method & Advantages & Disadvantages \\
\hline SLA & High printing resolution [250] & $\begin{array}{l}\text { Too long printing time [250] } \\
\text { Strict reinforce solvent option for } \\
\text { both graphene and SLA resin } \\
\text { [235] }\end{array}$ \\
& $\begin{array}{l}\text { Complicated self make filament } \\
\text { FDM }\end{array}$ & $\begin{array}{l}\text { Fast printing time [232] } \\
\text { Low cost [232] }\end{array}$ \\
& Market available filament [10] & $\begin{array}{l}\text { Spration process [231] } \\
\text { printed product [232] }\end{array}$
\end{tabular}


Required secondary loading Low cost [246] process [246, 247]

Can achieve binder-free GA Required the use of additive [242] monolith printing with proper with harsh additive removal rheological properties [245] process [13]

\section{Conclusion and perspective}

In the past two decades, 2D and 3D graphene products synthesized by different methods have played an important role in green energy research. They exhibited strong activity in green energy research, like fuel cell catalytic electrodes, LIBs, supercapacitor electrode, and photovoltaics (DSSCs and water splitting cell) research. However, most of these works were still at the fundamental activity analysis level. Performance evaluation of such materials in the real device scale, such as metal-air battery and coin cell scale, still occupy a small portion in total. Another challenge involves the graphene-based materials development in green energy research due to the limitation arising from the traditional synthesis and device fabrication techniques, such as (1) complicated procedure for 'bottom-up' CVD GF synthesis and the risk of uneven distribution of loaded catalyst in the 3D GA array by traditional chemical reduction or hydrothermal reactions; (2) shape and size of the GA and GA/NF products was controlled by the shape and size of reactor being used; (3) complicated process for the removal of supporting materials from the CVD synthesized GF; and (4) and the use of polymer binder for the electrode fabrication in the traditional electrochemical research (fuel cell, supercapacitor, LIBs, photovoltaic, etc.), which results in the low electrode activity and even high cost of electrode production. All these shortcomings limit the transfer of these techniques from laboratory scale to industrial scale. As a result, importing new technology has become an alternative to overcome the problem. Application of $3 \mathrm{D}$ printing technology has provided a possible solution for the size- and shape-customized graphene products synthesis for the commercialization of the graphene-based energy devices materials, especially the binder-free 3D GA based products. It is because besides the limitations on the 
desirable design reactor for the size and shape customized binder-free free-standing GA based electrode material synthesis mentioned previously, bulk 3D GA based materials synthesized by traditional chemical reduction or hydrothermal reactions have another risk of uneven distribution of loaded catalyst (especially $\mathrm{M}, \mathrm{MO}_{\mathrm{x}}$, and dissolved polymer) in the 3D GA array due to the lengthy stationary reaction. This may result in undesirable reactivity of the electrode produced from current synthesis methods. In addition, the effect of foreign material $\left(\mathrm{M}, \mathrm{MO}_{\mathrm{x}}\right.$, dissolved polymer) loading level to the electrochemical activity of the foreign materials loaded 3D printed graphene in battery research has also not been fully explored yet. Re-aggregation of GO into the graphitic structure may also take place throughout the traditional self-assembly reaction which may reduce the porosity of the GA products, affecting the electron transfer within the GA array. Conducting polymeric additive used in time-consuming FDM and SLA printed 3D graphene structures production may also cause problems in the printed electrode due to the masking of the specific active sites on the catalyst surface by the additives. All these barriers are needed to be solved for the future establishment of low-cost graphene-based electrode ink with optimized material loading for large-scale production. Merging of DIW 3D printing in the cooling tank method with the use of direct foreign materials/GO ink, and taking the advantage of binder-free battery electrode materials probably can: (1) solve the problem of uneven distribution of loaded materials in the GA array due to sedimentation during the GA self-assembly reaction; (2) achieve a controllable porosity in the GA array with the optimized foreign materials loading level for maximizing the battery and solar cell device performance; and (3) reduce production cost due to the elimination of extra additives and reactor free process throughout the synthesis. In short, DIW 3D printed binder-free GA-based electrode synthesis with uniform catalyst distribution should be carefully investigated in order to bring these products to commercialize at the device level. 
Acknowledgment

This work described in this paper was substantially supported by the National Natural Science Foundation of China (NSFC) and the Research Grants Council (RGC) of Hong Kong Joint Research Scheme (No. 51561165015 and No.N_HKU718/15), NSFC (21677179), the Guangdong Special Fund for Science \& Technology Development (Hong Kong Technology Cooperation Funding Scheme) (No. 2016A050503022), the Innovation Platform Construction of Guangdong and Hong Kong (No. 2017B050504001), Guangzhou Science and Technology Project (No. 201504301654288), the Key Fundamental Research Fund for the Central Universities (17lgjc17) and the National Key Research and Development Program of China (No. 2016YFC0204800), UK Engineering and Physical Sciences Research Council (EPSRC) via grant number EP/R012164/2 and EP/S000933/1, and the Royal Society via grant number $\mathrm{NAF} \backslash \mathrm{R} 1 \backslash 180146$.

\section{References:}

[1] Allen MJ, Tung VC, Kaner RB. Honeycomb Carbon: A Review of Graphene. Chem Rev. 2010;110:132-45.

[2] Rao CNR, Sood AK, Subrahmanyam KS, Govindaraj A. Graphene: The New TwoDimensional Nanomaterial. Ange Chem Int Ed. 2009;48:7752-77.

[3] Zhu YW, Murali S, Cai WW, Li XS, Suk JW, Potts JR, et al. Graphene and Graphene Oxide: Synthesis, Properties, and Applications. Adv Mater. 2010;22:3906-24.

[4] Balandin AA, Ghosh S, Bao W, Calizo I, Teweldebrhan D, Miao F, et al. Superior thermal conductivity of single-layer graphene. Nano Lett. 2008;8:902-7.

[5] Lee C, Wei X, Kysar JW, Hone J. Measurement of the elastic properties and intrinsic strength of monolayer graphene. Science. 2008;321:385-8.

[6] Stoller MD, Park S, Zhu Y, An J, Ruoff RS. Graphene-Based Ultracapacitors. Nano Lett. 2008;8:3498-502.

[7] Wang CW, Zhao Z, Li XF, Yan R, Wang J, Li AN, et al. Three-Dimensional Framework of Graphene Nanomeshes Shell/ $/ \mathrm{Co}_{3} \mathrm{O}_{4}$ Synthesized as Superior Bifunctional Electrocatalyst for Zinc-Air Batteries. ACS Appl Mater Interfaces. 2017;9:41273-83.

[8] Sun HT, Mei L, Liang JF, Zhao ZP, Lee C, Fei HL, et al. Three-dimensional holeygraphene/niobia composite architectures for ultrahigh-rate energy storage. Science. 2017;356:599-604.

[9] Meng JK, Suo Y, Li J, Zheng GP, Liu YS, Zhang JM, et al. Nitrogen-doped graphene aerogels as anode materials for lithium-ion battery: Assembly and electrochemical properties. Mater Lett. 2015;160:392-6. 
[10] Foster CW, Down MP, Zhang Y, Ji XB, Rowley-Neale SJ, Smith GC, et al. 3D Printed Graphene Based Energy Storage Devices. Sci Rep. 2017;7:42233.

[11] Vernardou D, Vasilopoulos KC, Kenanakis G. 3D printed graphene-based electrodes with high electrochemical performance. Appl Phys A. 2017;123:623.

[12] Tang X, Zhou H, Cai Z, Cheng D, He P, Xie P, et al. Generalized 3D Printing of Graphene-Based Mixed-Dimensional Hybrid Aerogels. ACS Nano. 2018;12:3502-11.

[13] Wang ZS, Zhang QE, Long SC, Luo YX, Yu PK, Tan ZB, et al. Three-Dimensional Printing of Polyaniline/Reduced Graphene Oxide Composite for High-Performance Planar Supercapacitor. ACS Appl Mater Interfaces. 2018;10:10437-44.

[14] Tuan Sang T, Dutta NK, Choudhury NR. Graphene inks for printed flexible electronics: Graphene dispersions, ink formulations, printing techniques and applications. Adv Colloid Interface Sci. 2018;261:41-61.

[15] Stafford J, Patapas A, Uzo N, Matar OK, Petit C. Towards scale-up of graphene production via nonoxidizing liquid exfoliation methods. AIChE J. 2018;64:3246-76.

[16] Yang Y, Han C, Jiang B, Iocozzia J, He C, Shi D, et al. Graphene-based materials with tailored nanostructures for energy conversion and storage. Mater Sci Eng R-Rep. 2016;102:172 .

[17] Ferrari AC, Bonaccorso F, Fal'ko V, Novoselov KS, Roche S, Boggild P, et al. Science and technology roadmap for graphene, related two-dimensional crystals, and hybrid systems. Nanoscale. 2015;7:4598-810.

[18] Li XS, Cai WW, An JH, Kim S, Nah J, Yang DX, et al. Large-Area Synthesis of HighQuality and Uniform Graphene Films on Copper Foils. Science. 2009;324:1312-4.

[19] Kairi MI, Khavarian M, Abu Bakar S, Vigolo B, Mohamed AR. Recent trends in graphene materials synthesized by CVD with various carbon precursors. J Mater Sci. 2018;53:851-79.

[20] Shang N, Papakonstantinou P, Wang P, Ravi S, Silva P. Platinum Integrated Graphene for Methanol Fuel Cells. J Phys Chem C. 2010;114:15837-41.

[21] Reddy ALM, Srivastava A, Gowda SR, Gullapalli H, Dubey M, Ajayan PM. Synthesis Of Nitrogen-Doped Graphene Films For Lithium Battery Application. ACS Nano. 2010;4:6337-42.

[22] Tang B, Hu G, Gao H, Shi Z. Three-dimensional graphene network assisted high performance dye sensitized solar cells. J Power Sources. 2013;234:60-8.

[23] Patil UM, Sohn JS, Kulkarni SB, Park HG, Jung Y, Gurav KV, et al. A facile synthesis of hierarchical alpha- $\mathrm{MnO}_{2}$ nanofibers on 3D-graphene foam for supercapacitor application. Mater Lett. 2014;119:135-9.

[24] Madrigal-Monge G, Chaves-Villareal C. CVD growth of $\mathrm{ZnO}$ nanorods in situ on graphene and the study of its application as photoanode for solar cells. Tecnologia En Marcha. 2017;30:104-18.

[25] Ruan G, Sun Z, Peng Z, Tour JM. Growth of Graphene from Food, Insects, and Waste. ACS Nano. 2011;5:7601-7.

[26] Ray AK, Sahu RK, Rajinikanth V, Bapari H, Ghosh M, Paul P. Preparation and characterization of graphene and $\mathrm{Ni}$-decorated graphene using flower petals as the precursor material. Carbon. 2012;50:4123-9.

[27] Sun Z, Yan Z, Yao J, Beitler E, Zhu Y, Tour JM. Growth of graphene from solid carbon sources. Nature. 2010;468:549-52.

[28] Sharma S, Kalita G, Hirano R, Shinde SM, Papon R, Ohtani H, et al. Synthesis of graphene crystals from solid waste plastic by chemical vapor deposition. Carbon. 2014;72:66-73.

[29] Ji J, Li Y, Peng W, Zhang G, Zhang F, Fan X. Advanced Graphene-Based Binder-Free Electrodes for High-Performance Energy Storage. Adv Mater. 2015;27:5264-79. 
[30] Hummers WS, Offeman RE. Preparation of graphitic oxide. J Am Chem Soc. 1958;80:1339.

[31] Kovtyukhova NI, Ollivier PJ, Martin BR, Mallouk TE, Chizhik SA, Buzaneva EV, et al. Layer-by-layer assembly of ultrathin composite films from micron-sized graphite oxide sheets and polycations. Chem Mat. 1999;11:771-8.

[32] Chen J, Sheng KX, Luo PH, Li C, Shi GQ. Graphene Hydrogels Deposited in Nickel Foams for High-Rate Electrochemical Capacitors. Adv Mater. 2012;24:4569-73.

[33] Ye SB, Feng JC, Wu PY. Deposition of Three-Dimensional Graphene Aerogel on Nickel Foam as a Binder-Free Supercapacitor Electrode. ACS Appl Mater Interfaces. 2013;5:71229.

[34] Wang P-Q, Bai Y, Luo P-Y, Liu J-Y. Graphene- $\mathrm{WO}_{3}$ nanobelt composite: Elevated conduction band toward photocatalytic reduction of $\mathrm{CO}_{2}$ into hydrocarbon fuels. Catal Commun. 2013;38:82-5.

[35] Morales-Torres S, Pastrana-Martinez LM, Figueiredo JL, Faria JL, Silva AMT. Graphene oxide-P25 photocatalysts for degradation of diphenhydramine pharmaceutical and methyl orange dye. Appl Surf Sci. 2013;275:361-8.

[36] Sun W, Lu X, Tong Y, Zhang Z, Lei J, Nie G, et al. Fabrication of highly dispersed palladium/graphene oxide nanocomposites and their catalytic properties for efficient hydrogenation of p-nitrophenol and hydrogen generation. Int $\mathbf{J}$ Hydrog Energy. 2014;39:9080-6.

[37] Nagaraju DH, Suresh GS. Green Chemistry Route to the Synthesis of Palladium Nanoparticles on Reduced Graphene Oxide for Ethanol Fuel Cells Applications. ECS Electrochem Lett. 2012;1:F21-F3.

[38] Sui Z, Zhang X, Lei Y, Luo Y. Easy and green synthesis of reduced graphite oxidebased hydrogels. Carbon. 2011;49:4314-21.

[39] Zhang L, Li N, Jiu H, Qi G, Huang Y. ZnO-reduced graphene oxide nanocomposites as efficient photocatalysts for photocatalytic reduction of $\mathrm{CO}_{2}$. Ceram Int. 2015;41:6256-62.

[40] Wang P, Wang J, Wang X, Yu H, Yu J, Lei M, et al. One-step synthesis of easyrecycling $\mathrm{TiO}_{2}-\mathrm{rGO}$ nanocomposite photocatalysts with enhanced photocatalytic activity. Appl Catal B-Environ. 2013;132-133:452-9.

[41] Marcano DC, Kosynkin DV, Berlin JM, Sinitskii A, Sun Z, Slesarev A, et al. Improved Synthesis of Graphene Oxide. ACS Nano. 2010;4:4806-14.

[42] Zhang X, Sui Z, Xu B, Yue S, Luo Y, Zhan W, et al. Mechanically strong and highly conductive graphene aerogel and its use as electrodes for electrochemical power sources. J Mater Chem. 2011;21:6494-7.

[43] Kim Y, Noh Y, Lim EJ, Lee S, Choi SM, Kim WB. Star-shaped Pd@Pt core-shell catalysts supported on reduced graphene oxide with superior electrocatalytic performance. J Mater Chem A. 2014;2:6976-86.

[44] Lim EJ, Kim Y, Choi SM, Lee S, Noh Y, Kim WB. Binary PdM catalysts (M=Ru, Sn, or Ir) over a reduced graphene oxide support for electro-oxidation of primary alcohols (methanol, ethanol, 1-propanol) under alkaline conditions. J Mater Chem A. 2015;3:5491500 .

[45] Kim SH, Jeong GH, Choi D, Yoon S, Jeon HB, Lee SM, et al. Synthesis of noble metal/graphene nanocomposites without surfactants by one-step reduction of metal salt and graphene oxide. J Colloid Interf Sci. 2013;389:85-90.

[46] Chen X, Su B, Wu G, Yang CJ, Zhuang Z, Wang X, et al. Platinum nanoflowers supported on graphene oxide nanosheets: their green synthesis, growth mechanism, and advanced electrocatalytic properties for methanol oxidation. J Mater Chem. 2012;22:11284-9. 
[47] Sawangphruk M, Krittayavathananon A, Chinwipas N. Ultraporous palladium on flexible graphene-coated carbon fiber paper as high-performance electro-catalysts for the electro-oxidation of ethanol. J Mater Chem A. 2013;1:1030-4.

[48] Yang J, Tian C, Wang L, Fu H. An effective strategy for small-sized and highlydispersed palladium nanoparticles supported on graphene with excellent performance for formic acid oxidation. J Mater Chem. 2011;21:3384-90.

[49] Yang X, Yang QD, Xu J, Lee CS. Bimetallic PtPd nanoparticles on Nafion-graphene film as catalyst for ethanol electro-oxidation. J Mater Chem. 2012;22:8057-62.

[50] Eigler S, Enzelberger-Heim M, Grimm S, Hofmann P, Kroener W, Geworski A, et al. Wet Chemical Synthesis of Graphene. Adv Mater. 2013;25:3583-7.

[51] Eigler S, Dotzer C, Hirsch A, Enzelberger M, Muller P. Formation and Decomposition of $\mathrm{CO}_{2}$ Intercalated Graphene Oxide. Chem Mat. 2012;24:1276-82.

[52] Kim S, Zhou S, Hu YK, Acik M, Chabal YJ, Berger C, et al. Room-temperature metastability of multilayer graphene oxide films. Nat Mater. 2012;11:544-9.

[53] Eigler S, Dotzer C, Hirsch A. Visualization of defect densities in reduced graphene oxide. Carbon. 2012;50:3666-73.

[54] Yang S, Zhuo K, Sun D, Wang X, Wang J. Preparation of graphene by exfoliating graphite in aqueous fulvic acid solution and its application in corrosion protection of aluminum. J Colloid Interf Sci. 2019;543:263-72.

[55] Tian J, Guo L, Yin X, Wu W. The liquid-phase preparation of graphene by shear exfoliation with graphite oxide as a dispersant. Mater Chem Phys. 2019;223:1-8.

[56] Ahmed MS, Kim YB. 3D graphene preparation via covalent amide functionalization for efficient metal-free electrocatalysis in oxygen reduction. Sci Rep. 2017;7:43279.

[57] Luan VH, Tien HN, Hoa LT, Hien NTM, Oh E-S, Chung J, et al. Synthesis of a highly conductive and large surface area graphene oxide hydrogel and its use in a supercapacitor. $\mathrm{J}$ Mater Chem A. 2013;1:208-11.

[58] Zhang L, Shi G. Preparation of Highly Conductive Graphene Hydrogels for Fabricating Supercapacitors with High Rate Capability. J Phys Chem C. 2011;115:17206-12.

[59] Hu H, Zhao Z, Wan W, Gogotsi Y, Qiu J. Ultralight and Highly Compressible Graphene Aerogels. Adv Mater. 2013;25:2219-23.

[60] Xu Z, Zhang Y, Li P, Gao C. Strong, Conductive, Lightweight, Neat Graphene Aerogel Fibers with Aligned Pores. ACS Nano. 2012;6:7103-13.

[61] Zhu H, Wang JT, Liu XL, Zhu XM. Three-dimensional porous graphene supported Ni nanoparticles with enhanced catalytic performance for Methanol electrooxidation. Int $\mathrm{J}$ Hydrog Energy. 2017;42:11206-14.

[62] Si W, Wu X, Zhou J, Guo F, Zhou S, Chu H, et al. Reduced graphene oxide aerogel with high-rate supercapacitive performance in aqueous electrolytes. Nanoscale Res Lett. 2013;8:247.

[63] Worsley MA, Pauzauskie PJ, Olson TY, Biener J, Satcher JH, Jr., Baumann TF. Synthesis of Graphene Aerogel with High Electrical Conductivity. J Am Chem Soc. 2010;132:14067-9.

[64] Worsley MA, Charnvanichborikarn S, Montalvo E, Shin SJ, Tylski ED, Lewicki JP, et al. Toward Macroscale, Isotropic Carbons with Graphene-Sheet-Like Electrical and Mechanical Properties. Adv Funct Mater. 2014;24:4259-64.

[65] Sheng K-x, Xu Y-x, Li C, Shi G-q. High-performance self-assembled graphene hydrogels prepared by chemical reduction of graphene oxide. New Carbon Mater. 2011;26:915.

[66] Wang ZY, Liu X, Shen X, Han NM, Wu Y, Zheng QB, et al. An Ultralight Graphene Honeycomb Sandwich for Stretchable Light-Emitting Displays. Adv Funct Mater. 2018;28:1707043. 
[67] Chang JH, Hung YH, Luo XF, Huang CH, Jung SM, Chang JK, et al. The hierarchical porosity of a three-dimensional graphene electrode for binder-free and high performance supercapacitors. RSC Adv. 2016;6:8384-94.

[68] Yang J, Zhang EW, Li XF, Yu YH, Qu J, Yu ZZ. Direct Reduction of Graphene Oxide by Ni Foam as a High-Capacitance Supercapacitor Electrode. ACS Appl Mater Interfaces. 2016;8:2297-305.

[69] Zheng CL, Zhang JL, Zhang Q, You B, Chen GL. Three dimensional Ni foam-supported graphene oxide for binder-free pseudocapacitor. Electrochim Acta. 2015;152:216-21.

[70] Xie YB, Zhan YY. Electrochemical capacitance of porous reduced graphene oxide/nickel foam. J Porous Materials. 2015;22:403-12.

[71] Huang HF, Xu LQ, Tang YM, Tang SL, Du YW. Facile synthesis of nickel network supported three-dimensional graphene gel as a lightweight and binder-free electrode for high rate performance supercapacitor application. Nanoscale. 2014;6:2426-33.

[72] Chen HS, Liang YT, Chen TY, Tseng YC, Liu CW, Chung SR, et al. Graphenesupported Pt and PtPd nanorods with enhanced electrocatalytic performance for the oxygen reduction reaction. Chem Commun. 2014;50:11165-8.

[73] Xie BB, Zhang Y, Zhang RJ. Coassembly and high ORR performance of monodisperse Pt nanocrystals with a mesopore-rich nitrogen-doped graphene aerogel. J Mater Chem A. 2017;5:17544-8.

[74] Ghosh S, Holade Y, Remita H, Servat K, Beaunier P, Hagege A, et al. One-pot synthesis of reduced graphene oxide supported gold-based nanomaterials as robust nanocatalysts for glucose electrooxidation. Electrochim Acta. 2016;212:864-75.

[75] Kabir S, Serov A, Artyushkova K, Atanassov P. Nitrogen-Doped Three-Dimensional Graphene-Supported Palladium Nanocomposites: High-Performance Cathode Catalysts for Oxygen Reduction Reactions. ACS Catal. 2017;7:6609-18.

[76] Li SN, Zhai YN, Zhang XY, MacFarlane DR. Surfactant-Free Synthesis of GrapheneSupported PdCu Nanocrystals with High Alloying Degree as Highly Active Catalyst for Formic Acid Electrooxidation. Adv Mater Interfaces. 2017;4:1700227.

[77] Li ZS, Li YY, He CY, Shen PK. Bimetallic PtAg alloyed nanoparticles and 3-D mesoporous graphene nanosheet hybrid architectures for advanced oxygen reduction reaction electrocatalysts. J Mater Chem A. 2017;5:23158-69.

[78] Yu M, Wu XK, Zhang JD, Meng YB, Ma YX, Liu JH, et al. Platinum nanoparticlesloaded holey reduced graphene oxide framework as freestanding counter electrodes of dye sensitized solar cells and methanol oxidation catalysts. Electrochim Acta. 2017;258:485-94.

[79] Feng J-X, Zhang Q-L, Wang A-J, Wei J, Chen J-R, Feng J-J. Caffeine-assisted facile synthesis of platinum@palladium core-shell nanoparticles supported on reduced graphene oxide with enhanced electrocatalytic activity for methanol oxidation. Electrochim Acta. 2014;142:343-50.

[80] Wu K, Zhang Q, Sun D, Zhu X, Chen Y, Lu T, et al. Graphene-supported Pd-Pt alloy nanoflowers: In situ growth and their enhanced electrocatalysis towards methanol oxidation. Int J Hydrog Energy. 2015;40:6530-7.

[81] Li N, Tang S, Meng X. Facile Synthesis of Bimetallic Au@Pd Nanoparticles with Coreshell Structures on Graphene Nanosheets. J Mater Sci Technol. 2014;30:1071-7.

[82] Li S-S, Yu J, Hu Y-Y, Wang A-J, Chen J-R, Feng J-J. Simple synthesis of hollow Pt-Pd nanospheres supported on reduced graphene oxide for enhanced methanol electrooxidation. $\mathrm{J}$ Power Sources. 2014;254:119-25.

[83] Zhang Y, Chang G, Shu H, Oyama M, Liu X, He Y. Synthesis of Pt-Pd bimetallic nanoparticles anchored on graphene for highly active methanol electro-oxidation. J Power Sources. 2014;262:279-85. 
[84] Zhang L-R, Zhao J, Li M, Ni H-T, Zhang J-L, Feng X-M, et al. Preparation of graphene supported nickel nanoparticles and their application to methanol electrooxidation in alkaline medium. New J Chem. 2012;36:1108-13.

[85] Liao C-S, Liao C-T, Tso C-Y, Shy H-J. Microwave-polyol synthesis and electrocatalytic performance of Pt/graphene nanocomposites. Mater Chem Phys. 2011;130:270-4.

[86] Qiu J-D, Wang G-C, Liang R-P, Xia X-H, Yu H-W. Controllable Deposition of Platinum Nanoparticles on Graphene As an Electrocatalyst for Direct Methanol Fuel Cells. J Phys Chem C. 2011;115:15639-45.

[87] Li Y, Gao W, Ci L, Wang C, Ajayan PM. Catalytic performance of Pt nanoparticles on reduced graphene oxide for methanol electro-oxidation. Carbon. 2010;48:1124-30.

[88] Zang J, Wang Y, Bian L, Zhang J, Meng F, Zhao Y, et al. Graphene growth on nanodiamond as a support for a Pt electrocatalyst in methanol electro-oxidation. Carbon. 2012;50:3032-8.

[89] Huang H, Chen H, Sun D, Wang X. Graphene nanoplate-Pt composite as a high performance electrocatalyst for direct methanol fuel cells. J Power Sources. 2012;204:46-52.

[90] Liu J, Zhou H, Wang Q, Zeng F, Kuang Y. Reduced graphene oxide supported palladium-silver bimetallic nanoparticles for ethanol electro-oxidation in alkaline media. $\mathrm{J}$ Mater Sci. 2012;47:2188-94.

[91] Li Y, Tang L, Li J. Preparation and electrochemical performance for methanol oxidation of Pt/graphene nanocomposites. Electrochem Commun. 2009;11:846-9.

[92] Dong Q, Zhao Y, Han X, Wang Y, Liu M, Li Y. Pd/Cu bimetallic nanoparticles supported on graphene nanosheets: Facile synthesis and application as novel electrocatalyst for ethanol oxidation in alkaline media. Int J Hydrog Energy. 2014;39:14669-79.

[93] Chen XM, Cai ZX, Chen X, Oyama M. Green synthesis of graphene-PtPd alloy nanoparticles with high electrocatalytic performance for ethanol oxidation. J Mater Chem A. 2014;2:315-20.

[94] Zhang Y, Huang Q, Chang G, Zhang Z, Xia T, Shu H, et al. Controllable synthesis of palladium nanocubes/reduced graphene oxide composites and their enhanced electrocatalytic performance. J Power Sources. 2015;280:422-9.

[95] Peng C, Liu M, Hu Y, Yang W, Guo J, Zheng Y. $\mathrm{Pd}_{\mathrm{x}} \mathrm{Ag}_{\mathrm{y}}$ alloy nanoparticles supported on reduced graphene oxide as efficient electrocatalyst for ethanol oxidation in alkaline medium. RSC Adv. 2015;5:49899-903.

[96] Bin D, Yang B, Ren F, Zhang K, Yang P, Du Y. Facile synthesis of PdNi nanowire networks supported on reduced graphene oxide with enhanced catalytic performance for formic acid oxidation. J Mater Chem A. 2015;3:14001-6.

[97] Yang S, Dong J, Yao Z, Shen C, Shi X, Tian Y, et al. One-Pot Synthesis of GrapheneSupported Monodisperse Pd Nanoparticles as Catalyst for Formic Acid Electro-oxidation. Sci Rep. 2014;4:04501.

[98] Huang HJ, Wang X. Pd nanoparticles supported on low-defect graphene sheets: for use as high-performance electrocatalysts for formic acid and methanol oxidation. J Mater Chem. 2012;22:22533-41.

[99] Li F, Guo Y, Li R, Wu F, Liu Y, Sun X, et al. A facile method to synthesize supported $\mathrm{Pd}-\mathrm{Au}$ nanoparticles using graphene oxide as the reductant and their extremely high electrocatalytic activity for the electrooxidation of methanol and ethanol. J Mater Chem A. 2013;1:6579-87.

[100] Li L, Chen M, Huang G, Yang N, Zhang L, Wang H, et al. A green method to prepare Pd-Ag nanoparticles supported on reduced graphene oxide and their electrochemical catalysis of methanol and ethanol oxidation. J Power Sources. 2014;263:13-21.

[101] Na H, Zhang L, Qiu H, Wu T, Chen M, Yang N, et al. A two step method to synthesize palladium-copper nanoparticles on reduced graphene oxide and their extremely high 
electrocatalytic activity for the electrooxidation of methanol and ethanol. J Power Sources. 2015;288:160-7.

[102] Gao L, Yue W, Tao S, Fan L. Novel Strategy for Preparation of Graphene-Pd, Pt Composite, and Its Enhanced Electrocatalytic Activity for Alcohol Oxidation. Langmuir. 2013;29:957-64.

[103] Nagaraju DH, Devaraj S, Balaya P. Palladium nanoparticles anchored on graphene nanosheets: Methanol, ethanol oxidation reactions and their kinetic studies. Mater Res Bull. 2014;60:150-7.

[104] Li S-S, Zheng J-N, Ma X, Hu Y-Y, Wang A-J, Chen J-R, et al. Facile synthesis of hierarchical dendritic PtPd nanogarlands supported on reduced graphene oxide with enhanced electrocatalytic properties. Nanoscale. 2014;6:5708-13.

[105] Lv J-J, Li S-S, Wang A-J, Mei L-P, Feng J-J, Chen J-R, et al. One-pot synthesis of monodisperse palladium-copper nanocrystals supported on reduced graphene oxide nanosheets with improved catalytic activity and methanol tolerance for oxygen reduction reaction. J Power Sources. 2014;269:104-10.

[106] Li S-S, Lv J-J, Teng L-N, Wang A-J, Chen J-R, Feng J-J. Facile Synthesis of PdPt@Pt Nanorings Supported on Reduced Graphene Oxide with Enhanced Electrocatalytic Properties. ACS Appl Mater Interfaces. 2014;6:10549-55.

[107] Lv J-J, Wisitruangsakul N, Feng J-J, Luo J, Fang K-M, Wang A-J. Biomoleculeassisted synthesis of porous PtPd alloyed nanoflowers supported on reduced graphene oxide with highly electrocatalytic performance for ethanol oxidation and oxygen reduction. Electrochim Acta. 2015;160:100-7.

[108] Huang C-C, Pu N-W, Wang C-A, Huang J-C, Sung Y, Ger M-D. Hydrogen storage in graphene decorated with $\mathrm{Pd}$ and $\mathrm{Pt}$ nano-particles using an electroless deposition technique. Sep Pur Technol. 2011;82:210-5.

[109] $\mathrm{Wu} \mathrm{H}$, Wexler D, Liu H. Durability investigation of graphene-supported Pt nanocatalysts for PEM fuel cells. J Solid State Electrochem. 2011;15:1057-62.

[110] Shao Y, Zhang S, Wang C, Nie Z, Liu J, Wang Y, et al. Highly durable graphene nanoplatelets supported $\mathrm{Pt}$ nanocatalysts for oxygen reduction. J Power Sources. 2010;195:4600-5.

[111] Ren L, Hui KS, Hui KN. Self-assembled free-standing three-dimensional nickel nanoparticle/graphene aerogel for direct ethanol fuel cells. J Mater Chem A. 2013;1:5689-94.

[112] Li J, Liu C-y, Liu Y. Au/graphene hydrogel: synthesis, characterization and its use for catalytic reduction of 4-nitrophenol. J Mater Chem. 2012;22:8426-30.

[113] Liu M, Peng C, Yang W, Guo J, Zheng Y, Chen P, et al. Pd nanoparticles supported on three-dimensional graphene aerogels as highly efficient catalysts for methanol electrooxidation. Electrochim Acta. 2015;178:838-46.

[114] Zhao H, Yang J, Wang L, Tian C, Jiang B, Fu H. Fabrication of a palladium nanoparticle/graphene nanosheet hybrid via sacrifice of a copper template and its application in catalytic oxidation of formic acid. Chem Commun. 2011;47:2014-6.

[115] Wang Y, Zhao Y, He WT, Yin J, Su YQ. Palladium nanoparticles supported on reduced graphene oxide: Facile synthesis and highly efficient electrocatalytic performance for methanol oxidation. Thin Solid Films. 2013;544:88-92.

[116] Chen S, Zhu JW, Qiu L, Li D, Wang X. Facile Fabrication of Nanoparticles Confined in Graphene Films and Their Electrochemical Properties. Chem Eur J. 2013;19:7631-6.

[117] Hong L, Hao Y, Yang Y, Yuan J, Niu L. Synthesis of graphene-supported onedimensional nanoporous Pt based catalysts, and their enhanced performance on methanol electro-oxidation. Nanotechnol. 2015;26:045604. 
[118] Wang Q, Cui X, Guan W, Zheng W, Chen J, Zheng X, et al. Synthesis of flower-shape palladium nanostructures on graphene oxide for electrocatalytic applications. J Phys Chem Solids. 2013;74:1470-4.

[119] Sahu SC, Samantara AK, Dash A, Juluri RR, Sahu RK, Mishra BK, et al. Grapheneinduced Pd nanodendrites: A high performance hybrid nanoelectrocatalyst. Nano Res. 2013;6:635-43.

[120] Wojnicki M, Luty-Błocho M, Dobosz I, Grzonka J, Pacławski K, Kurzydłowski KJ, et al. Electro-Oxidation of Glucose in Alkaline Media on Graphene Sheets Decorated with Gold Nanoparticles. Mater Sci Appl. 2013;4:162-9.

[121] Bao Q, Hui KN, Hui KS, Wang Y, Hong X. Hydrothermal self-assembly and supercapacitive behaviors of $\mathrm{Co}$ (II) ion-modified graphene aerogels in $\mathrm{H}_{2} \mathrm{SO}_{4}$ electrolyte. Mater Res Bull. 2014;56:92-7.

[122] Yun S, Lee S, Shin C, Park S, Kwon SJ, Park HS. One-Pot Self-Assembled, Reduced Graphene Oxide/Palladium Nanoparticle Hybrid Aerogels for Electrocatalytic Applications. Electrochim Acta. 2015;180:902-8.

[123] Wang R, Wu Z, Chen C, Qin Z, Zhu H, Wang G, et al. Graphene-supported Au-Pd bimetallic nanoparticles with excellent catalytic performance in selective oxidation of methanol to methyl formate. Chem Commun. 2013;49:8250-2.

[124] Liu TY, Li CZ, Yuan Q. Facile Synthesis of PtCu Alloy/Graphene Oxide Hybrids as Improved Electrocatalysts for Alkaline Fuel Cells. ACS Omega. 2018;3:8724-32.

[125] Mahajan A, Banik S, Majumdar D, Bhattacharya SK. Anodic Oxidation of Butan-1-ol on Reduced Graphene Oxide-Supported Pd-Ag Nanoalloy for Fuel Cell Application. ACS Omega. 2019;4:4658-70.

[126] Ye SJ, Bui HT, Kim YY, Liao K, Cho KM, Jung HT, et al. Facile Synthesis of Composition-Controlled Graphene-Supported PtPd Alloy Nanocatalysts and Their Applications in Methanol Electro-Oxidation and Lithium-Oxygen Batteries. Chem Eur J. 2017;23:17136-43.

[127] Liang Y, Li Y, Wang $\mathrm{H}$, Zhou J, Wang J, Regier T, et al. $\mathrm{Co}_{3} \mathrm{O}_{4}$ nanocrystals on graphene as a synergistic catalyst for oxygen reduction reaction. Nat Mater. 2011;10:780-6.

[128] Wu J, Chen C, Hao Y, Wang C. Enhanced electrochemical performance of nano sheet $\mathrm{ZnO}$ /reduced graphene oxide composites as anode for lithium-ion batteries. Colloid Surf APhysicochem Eng Asp. 2015;468:17-21.

[129] Yan J, Fan ZJ, Wei T, Qian WZ, Zhang ML, Wei F. Fast and reversible surface redox reaction of graphene- $\mathrm{MnO}_{2}$ composites as supercapacitor electrodes. Carbon. 2010;48:382533.

[130] Wang Z, Sha J, Liu E, He C, Shi C, Li J, et al. A large ultrathin anatase $\mathrm{TiO}_{2}$ nanosheet/reduced graphene oxide composite with enhanced lithium storage capability. J Mater Chem A. 2014;2:8893-901.

[131] Chen Y-L, Hu Z-A, Chang Y-Q, Wang H-W, Zhang Z-Y, Yang Y-Y, et al. Zinc Oxide/Reduced Graphene Oxide Composites and Electrochemical Capacitance Enhanced by Homogeneous Incorporation of Reduced Graphene Oxide Sheets in Zinc Oxide Matrix. J Phys Chem C. 2011;115:2563-71.

[132] Bu IYY, Huang R. One-pot synthesis of $\mathrm{ZnO} /$ reduced graphene oxide nanocomposite for supercapacitor applications. Mater Sci Semiconductor Processing. 2015;31:131-8.

[133] El-Deen AG, Barakat NAM, Khalil KA, Motlak M, Kim HY. Graphene/SnO ${ }_{2}$ nanocomposite as an effective electrode material for saline water desalination using capacitive deionization. Ceram Int. 2014;40:14627-34.

[134] Yin H, Zhao S, Wan J, Tang H, Chang L, He L, et al. Three-Dimensional Graphene/Metal Oxide Nanoparticle Hybrids for High-Performance Capacitive Deionization of Saline Water. Adv Mater. 2013;25:6270-6. 
[135] Haldorai Y, Rengaraj A, Kwak CH, Huh YS, Han Y-K. Fabrication of nano $\mathrm{TiO}_{2} @$ graphene composite: Reusable photocatalyst for hydrogen production, degradation of organic and inorganic pollutants. Synth Metals. 2014;198:10-8.

[136] Liu C, Gao AM, Yi FY, Shu D, Yi H, Zhou XP, et al. Anchoring ultrafine $\mathrm{Co}_{3} \mathrm{O}_{4}$ grains on reduced oxide graphene by dual-template nanocasting strategy for high-energy solid state supercapacitor. Electrochim Acta. 2019;326:134965.

[137] Mussa Y, Ahmed F, Abuhimd H, Arsalan M, Alsharaeh E. Enhanced Electrochemical performance at high temperature of Cobalt Oxide/Reduced Graphene Oxide Nanocomposites and its application in lithium-ion batteries. Sci Rep. 2019;9:44.

[138] Jiang TT, Yin NQ, Bai ZM, Dai P, Yu XX, Wu MZ, et al. Wet chemical synthesis of S doped $\mathrm{Co}_{3} \mathrm{O}_{4}$ nanosheets/reduced graphene oxide and their application in dye sensitized solar cells. Appl Surf Sci. 2018;450:219-27.

[139] Gao P, Sun DD. Ultrasonic Preparation of Hierarchical Graphene-Oxide/ $/ \mathrm{TiO}_{2}$ Composite Microspheres for Efficient Photocatalytic Hydrogen Production. Chem Asian J. 2013;8:2779-86.

[140] Shu W, Liu Y, Peng Z, Chen K, Zhang C, Chen W. Synthesis and photovoltaic performance of reduced graphene oxide- $\mathrm{TiO}_{2}$ nanoparticles composites by solvothermal method. J Alloy Comp. 2013;563:229-33.

[141] Huang H, Fang J, Xia Y, Tao X, Gan Y, Du J, et al. Construction of sheet-belt hybrid nanostructures from one-dimensional mesoporous $\mathrm{TiO}_{2}(\mathrm{~B})$ nanobelts and graphene sheets for advanced lithium-ion batteries. J Mater Chem A. 2013;1:2495-500.

[142] Xiao Y, Qin J, Hu C, Cao M. P25/graphene nanocomposites as a high-performance anode material for lithium ion batteries. Mater Chem Phys. 2013;141:153-9.

[143] Xu CL, Liu Z, Wei T, Sheng LZ, Zhang LH, Chen L, et al. Mesoporous singlecrystalline $\mathrm{MnO}_{\mathrm{x}}$ nanofibers@graphene for ultra-high rate and long-life lithium-ion battery anodes. J Mater Chem A. 2018;6:24756-66.

[144] Chabu JM, Wang LQ, Tang FY, Zeng K, Sheng JP, Walle MD, et al. Synthesis of Three-Dimensional Nitrogen and Sulfur Dual-Doped Graphene Aerogels as an Efficient Metal-Free Electrocatalyst for the Oxygen Reduction Reaction. ChemEectroCem. 2017;4:1885-90.

[145] Sui Z-Y, Meng Y-N, Xiao P-W, Zhao Z-Q, Wei Z-X, Han B-H. Nitrogen-Doped Graphene Aerogels as Efficient Supercapacitor Electrodes and Gas Adsorbents. ACS Appl Mater Interfaces. 2015;7:1431-8.

[146] Chen P, Yang J-J, Li S-S, Wang Z, Xiao T-Y, Qian Y-H, et al. Hydrothermal synthesis of macroscopic nitrogen-doped graphene hydrogels for ultrafast supercapacitor. Nano Energy. 2013;2:249-56.

[147] Song X, Lin L, Rong M, Wang Y, Xie Z, Chen X. Mussel-inspired, ultralight, multifunctional 3D nitrogen-doped graphene aerogel. Carbon. 2014;80:174-82.

[148] Wu Z-S, Winter A, Chen L, Sun Y, Turchanin A, Feng X, et al. Three-Dimensional Nitrogen and Boron Co-doped Graphene for High-Performance All-Solid-State Supercapacitors. Adv Mater. 2012;24:5130-5.

[149] Zhou Y, Yen CH, Fu S, Yang G, Zhu C, Du D, et al. One-pot synthesis of B-doped three-dimensional reduced graphene oxide via supercritical fluid for oxygen reduction reaction. Green Chem. 2015;17:3552-60.

[150] Huang HF, Lei CL, Luo GS, Cheng ZZ, Li GX, Tang SL, et al. Facile synthesis of nitrogen-doped graphene on $\mathrm{Ni}$ foam for high-performance supercapacitors. J Mater Sci. 2016;51:6348-56.

[151] Zhang Y, Fugane K, Mori T, Niu L, Ye J. Wet chemical synthesis of nitrogen-doped graphene towards oxygen reduction electrocatalysts without high-temperateure pyrolysis. $\mathrm{J}$ Mater Chem. 2012;22:6575-80. 
[152] Han J, Cheon JY, Joo SH, Park S. Synthesis of boron and nitrogen co-doped graphene nano-platelets using a two-step solution process and catalytic properties for oxygen reduction reaction. Solid State Sci. 2014;33:1-5.

[153] Yeom D-Y, Jeon W, Tu NDK, Yeo SY, Lee S-S, Sung BJ, et al. High-concentration boron doping of graphene nanoplatelets by simple thermal annealing and their supercapacitive properties. Sci Rep. 2015;5:09817.

[154] Huynh Ngoc T, Kocabas C, Hur SH. One-step codoping of reduced graphene oxide using boric and nitric acid mixture and its use in metal-free electrocatalyst. Mater Lett. 2015;143:205-8.

[155] Huang HJ, Zhu JX, Zhang WY, Tiwary CS, Zhang JF, Zhang X, et al. Controllable Codoping of Nitrogen and Sulfur in Graphene for Highly Efficient Li-Oxygen Batteries and Direct Methanol Fuel Cells. Chem Mat. 2016;28:1737-45.

[156] Huo JH, Zheng P, Wang XF, Guo SW. Three-dimensional sulphur/nitrogen co-doped reduced graphene oxide as high-performance supercapacitor binder-free electrodes. Appl Surf Sci. 2018;442:575-80.

[157] Chen YJ, Liu ZE, Sun L, Lu ZW, Zhuo KL. Nitrogen and sulfur co-doped porous graphene aerogel as an efficient electrode material for high performance supercapacitor in ionic liquid electrolyte. J Power Sources. 2018;390:215-23.

[158] Khan M, Bin Yousaf A, Chen M, Wei C, Wu X, Huang N, et al. Mixed-phase Pd-Pt bimetallic alloy on graphene oxide with high activity for electrocatalytic applications. J Power Sources. 2015;282:520-8.

[159] Sun S, Zhang G, Gauquelin N, Chen N, Zhou J, Yang S, et al. Single-atom Catalysis Using Pt/Graphene Achieved through Atomic Layer Deposition. Sci Rep. 2013;3:1775.

[160] Fan LS, Zhang Y, Guo ZK, Sun B, Tian D, Feng YJ, et al. Hierarchical $\mathrm{Mn}_{3} \mathrm{O}_{4}$ Anchored on 3D Graphene Aerogels via C-O-Mn Linkage with Superior Electrochemical Performance for Flexible Asymmetric Supercapacitor. Chem Eur J. 2019;25:1-6.

[161] Wang $\mathrm{Y}$, Jin $\mathrm{YH}$, Jia MQ. Ultralong $\mathrm{Fe}_{3} \mathrm{O}_{4}$ nanowires embedded graphene aerogel composite anodes for lithium ion batteries. Mater Lett. 2018;228:395-8.

[162] Yu M, Huang Y, Li C, Zeng Y, Wang W, Li Y, et al. Building Three-Dimensional Graphene Frameworks for Energy Storage and Catalysis. Adv Funct Mater. 2015;25:324-30.

[163] Awasthi R, Singh RN. Graphene-supported Pd-Ru nanoparticles with superior methanol electrooxidation activity. Carbon. 2013;51:282-9.

[164] Tsang CHA, Hui KN, Hui KS, Ren L. Deposition of Pd/graphene aerogel on nickel foam as a binder-free electrode for direct electro-oxidation of methanol and ethanol. J Mater Chem A. 2014;2:17986-93.

[165] Tsang CHA, Hui KN, Hui KS. Electrooxidation of glucose by binder-free bimetallic $\mathrm{Pd}_{1} \mathrm{Pt}_{\mathrm{x}} /$ graphene aerogel/nickel foam composite electrodes with low metal loading in basic medium. Electrochim Acta. 2017;258:371-9.

[166] Pan F, Chen SM, Li YH, Tao ZC, Ye JL, Ni K, et al. 3D Graphene Films Enable Simultaneously High Sensitivity and Large Stretchability for Strain Sensors. Adv Funct Mater. 2018;28:1803221.

[167] Zheng L, Cheng XH, Ye PY, Shen LY, Wang Q, Zhang DL, et al. Low temperature growth of three-dimensional network of graphene for high-performance supercapacitor electrodes. Mater Lett. 2018;218:90-4.

[168] Hoa NV, Lamiel C, Nghia NH, Dat PA, Shim JJ. Different morphologies of $\mathrm{MnO}_{2}$ grown on the graphene@nickel foam electrode for supercapacitor application. Mater Lett. 2017;208:102-6.

[169] Fei H, Ye R, Ye G, Gong Y, Peng Z, Fan X, et al. Boron- and Nitrogen-Doped Graphene Quantum Dots/Graphene Hybrid Nanoplatelets as Efficient Electrocatalysts for Oxygen Reduction. ACS Nano. 2014;8:10837-43. 
[170] Zhou JH, Qi F, Chen YF, Wang ZG, Zheng BJ, Wang XQ. CVD-grown threedimensional sulfur-doped graphene as a binder-free electrocatalytic electrode for highly effective and stable hydrogen evolution reaction. J Mater Sci. 2018;53:7767-77.

[171] Tang B, Wang S, Zhang J, Wang Z, He Y, Huang W. Three-dimensional graphene monolith-based composite: superiority in properties and applications. Int Mater Rev. 2018;63:204-25.

[172] Lv W, Li Z, Deng Y, Yang Q-H, Kang F. Graphene-based materials for electrochemical energy storage devices: Opportunities and challenges. Energy Storage Mater. 2016;2:107-38.

[173] Bhuyan MSA, Uddin MN, Islam MM, Bipasha FA, Hossain SS. Synthesis of graphene. Int Nano Lett. 2016;6:65-83.

[174] Tahir AA, Ullah H, Sudhagar P, Teridi MAM, Devadoss A, Sundaram S. The Application of Graphene and Its Derivatives to Energy Conversion, Storage, and Environmental and Biosensing Devices. Chem Record. 2016;16:1591-634.

[175] Al Hassan MR, Sen A, Zaman T, Mostari MS. Emergence of graphene as a promising anode material for rechargeable batteries: a review. Mater Today Chem. 2019;11:225-43.

[176] Li X, Zhi L. Graphene hybridization for energy storage applications. Chem Soc Rev. 2018;47:3189-216.

[177] Ferrari AC, Bonaccorso F, Fal'ko V, Novoselov KS, Roche S, Boggild P, et al. Science and technology roadmap for graphene, related two-dimensional crystals, and hybrid systems. Nanoscale. 2015;7:4598-810.

[178] El-Kady MF, Shao Y, Kaner RB. Graphene for batteries, supercapacitors and beyond. Nat Rev Mater. 2016;1:16033.

[179] Wang Z, Gao H, Zhang Q, Liu Y, Chen J, Guo Z. Recent Advances in 3D Graphene Architectures and Their Composites for Energy Storage Applications. Small. 2019;15:1803858.

[180] Liu J-Y, Li X-X, Huang J-R, Li J-J, Zhou P, Liu J-H, et al. Three-dimensional graphene-based nanocomposites for high energy density Li-ion batteries. J Mater Chem A. 2017;5:5977-94.

[181] Yao X, Zhao Y. Three-Dimensional Porous Graphene Networks and Hybrids for Lithium-Ion Batteries and Supercapacitors. Chem. 2017;2:171-200.

[182] Shi QF, Diao GW, Mu SL. The electrocatalytic oxidation of glucose on the bimetallic $\mathrm{Au}-\mathrm{Ag}$ particles-modified reduced graphene oxide electrodes in alkaline solutions. Electrochim Acta. 2014;133:335-46.

[183] Rajkumar M, Devadas B, Chen S-M, Yeh P-C. Single step electrochemical fabrication of highly loaded palladium nanoparticles decorated chemically reduced graphene oxide and its electrocatalytic applications. Colloid Surf A-Physicochem Eng Asp. 2014;452:39-45.

[184] Chen Q-S, Xu Z-N, Peng S-Y, Chen Y-M, Lv D-M, Wang Z-Q, et al. One-step electrochemical synthesis of preferentially oriented (111) Pd nanocrystals supported on graphene nanoplatelets for formic acid electrooxidation. J Power Sources. 2015;282:471-8.

[185] Ojani R, Raoof J-B, Li MG, Valiollahi R. Pt-Co nanostructures electrodeposited on graphene nanosheets for methanol electrooxidation. J Power Sources. 2014;264:76-82.

[186] Chen C, Long M, Wu H, Cai W. One-step synthesis of Pt nanoparticles/reduced graphene oxide composite with enhanced electrochemical catalytic activity. Science ChinaChem. 2013;56:354-61.

[187] Liu S, Wang J, Zeng J, Ou J, Li Z, Liu X, et al. "Green" electrochemical synthesis of $\mathrm{Pt} /$ graphene sheet nanocomposite film and its electrocatalytic property. J Power Sources. 2010;195:4628-33. 
[188] Zhou Y-G, Chen J-J, Wang F-b, Sheng Z-H, Xia X-H. A facile approach to the synthesis of highly electroactive Pt nanoparticles on graphene as an anode catalyst for direct methanol fuel cells. Chem Commun. 2010;46:5951-3.

[189] Ashkarran A, Mohammadi B. ZnO nanoparticles decorated on graphene sheets through liquid arc discharge approach with enhanced photocatalytic performance under visible-light. Appl Surf Sci. 2015;342:112-9.

[190] Zhang Z, Ren L, Han W, Meng L, Wei X, Qi X, et al. One-pot electrodeposition synthesis of $\mathrm{ZnO} /$ graphene composite and its use as binder-free electrode for supercapacitor. Ceram Int. 2015;41:4374-80.

[191] Wang X, Li X, Zhang L, Yoon Y, Weber PK, Wang H, et al. N-Doping of Graphene Through Electrothermal Reactions with Ammonia. Science. 2009;324:768-71.

[192] Maiyalagan T, Dong XC, Chen P, Wang X. Electrodeposited Pt on three-dimensional interconnected graphene as a free-standing electrode for fuel cell application. J Mater Chem. 2012;22:5286-90.

[193] Hui KS, Hui KN, Dinh DA, Tsang CH, Cho YR, Zhou W, et al. Green synthesis of dimension-controlled silver nanoparticle-graphene oxide with in situ ultrasonication. Acta Materialia. 2014;64:326-32.

[194] Zheng J-N, Li S-S, Ma X, Chen F-Y, Wang A-J, Chen J-R, et al. Green synthesis of core-shell gold-palladium@palladium nanocrystals dispersed on graphene with enhanced catalytic activity toward oxygen reduction and methanol oxidation in alkaline media. J Power Sources. 2014;262:270-8.

[195] Neppolian B, Saez V, Gonzalez-Garcia J, Grieser F, Gomez R, Ashokkumar M. Sonochemical synthesis of graphene oxide supported Pt-Pd alloy nanocrystals as efficient electrocatalysts for methanol oxidation. J Solid State Electrochem. 2014;18:3163-71.

[196] Lee KG, Jeong J-M, Lee SJ, Yeom B, Lee M-K, Choi BG. Sonochemical-assisted synthesis of 3D graphene/nanoparticle foams and their application in supercapacitor. Ultrasonics Sonochem. 2015;22:422-8.

[197] Wei LG, Chen SS, Yang YL, Dong YL, Song WN, Fan RQ. Effect of Graphene/TiO 2 Composite Layer on the Performance of Dye-Sensitized Solar Cells. J Nanosci Nanotechnol. 2018;18:976-83.

[198] Wei LG, Wang P, Yang YL, Dong YL, Fan RQ, Song WN, et al. Enhanced performance of dye sensitized solar cells by using a reduced graphene oxide $/ \mathrm{TiO}_{2}$ blocking layer in the photoanode. Thin Solid Films. 2017;639:12-21.

[199] Siwach B, Mohan D, Jyoti D. To investigate opulence of graphene in ZnO/graphene nanocomposites based dye sensitized solar cells. J Mater Sci-Mater Electron. 2017;28:115008 .

[200] Ghorbani M, Abdizadeh H, Taheri M, Golobostanfard MR. Enhanced photoelectrochemical water splitting in hierarchical porous $\mathrm{ZnO} /$ Reduced graphene oxide nanocomposite synthesized by sol-gel method. Int J Hydrog Energy. 2018;43:7754-63.

[201] Kung CC, Lin PY, Xue YH, Akolkar R, Dai LM, Yu X, et al. Three dimensional graphene foam supported platinum-ruthenium bimetallic nanocatalysts for direct methanol and direct ethanol fuel cell applications. J Power Sources. 2014;256:329-35.

[202] Tsang CHA, Hui KN, Hui KS. Influence of $\mathrm{Pd}_{1} \mathrm{Pt}_{\mathrm{x}}$ alloy $\mathrm{NPs}$ on graphene aerogel/nickel foam as binder-free anodic electrode for electrocatalytic ethanol oxidation reaction. J Power Sources. 2019;413:98-106.

[203] Tsang CHA, Leung DYC. Use of Pd-Pt loaded graphene aerogel on nickel foam in direct ethanol fuel cell. Solid State Sci. 2018;75:21-6.

[204] Tsang CHA, Leung DYC. Pd-Pt loaded graphene aerogel on nickel foam composite as binder-free anode for a direct glucose fuel cell unit. Solid State Sci. 2017;71:123-9. 
[205] Wang S, Zhang L, Xia Z, Roy A, Chang DW, Baek J-B, et al. BCN Graphene as Efficient Metal-Free Electrocatalyst for the Oxygen Reduction Reaction. Angew Chem Int Ed. 2012;51:4209-12.

[206] Xiao J, Mei D, Li X, Xu W, Wang D, Graff GL, et al. Hierarchically Porous Graphene as a Lithium-Air Battery Electrode. Nano Lett. 2011;11:5071-8.

[207] Zhu JX, Yang D, Rui XH, Sim D, Yu H, Hng HH, et al. Facile Preparation of Ordered Porous Graphene-Metal Oxide@C Binder-Free Electrodes with High Li Storage Performance. Small. 2013;9:3390-7.

[208] Sahoo M, Sreena KP, Vinayan BP, Ramaprabhu S. Green synthesis of boron doped graphene and its application as high performance anode material in Li ion battery. Mater Res Bull. 2015;61:383-90.

[209] Jeong JH, Lee GW, Kim YH, Choi YJ, Roh KC, Kim KB. A holey graphene-based hybrid supercapacitor. Chem Eng J. 2019;378:122126.

[210] Radich JG, Kamat PV. Origin of Reduced Graphene Oxide Enhancements in Electrochemical Energy Storage. ACS Catal. 2012;2:807-16.

[211] Ning G, Fan Z, Wang G, Gao J, Qian W, Wei F. Gram-scale synthesis of nanomesh graphene with high surface area and its application in supercapacitor electrodes. Chem Commun. 2011;47:5976-8.

[212] Zhu Y, Murali S, Stoller MD, Ganesh KJ, Cai W, Ferreira PJ, et al. Carbon-Based Supercapacitors Produced by Activation of Graphene. Science. 2011;332:1537-41.

[213] Lim MB, Hu M, Manandhar S, Sakshaug A, Strong A, Riley L, et al. Ultrafast sol-gel synthesis of graphene aerogel materials. Carbon. 2015;95:616-24.

[214] Zhang LL, Zhao X, Stoller MD, Zhu Y, Ji H, Murali S, et al. Highly Conductive and Porous Activated Reduced Graphene Oxide Films for High-Power Supercapacitors. Nano Lett. 2012;12:1806-12.

[215] Fan Z, Zhao Q, Li T, Yan J, Ren Y, Feng J, et al. Easy synthesis of porous graphene nanosheets and their use in supercapacitors. Carbon. 2012;50:1699-703.

[216] Xu Y, Sheng K, Li C, Shi G. Self-Assembled Graphene Hydrogel via a One-Step Hydrothermal Process. ACS Nano. 2010;4:4324-30.

[217] Ramabadran U, Ryan G, Zhou X, Farhat S, Manciu F, Tong YG, et al. Reduced Graphene Oxide on Nickel Foam for Supercapacitor Electrodes. Mater. 2017;10:1295.

[218] Gao Z, Wang J, Li ZS, Yang WL, Wang B, Hou MJ, et al. Graphene Nanosheet $/ \mathrm{Ni}^{2+} / \mathrm{Al}^{3+}$ Layered Double-Hydroxide Composite as a Novel Electrode for a Supercapacitor. Chem Mat. 2011;23:3509-16.

[219] Wang Z, Zhang X, Wang JH, Zou LD, Liu ZT, Hao ZP. Preparation and capacitance properties of graphene/NiAl layered double-hydroxide nanocomposite. J Colloid Interf Sci. 2013;396:251-7.

[220] Yan L, Li RY, Li ZJ, Liu JK, Fang YJ, Wang GL, et al. Three-dimensional activated reduced graphene oxide nanocup/nickel aluminum layered double hydroxides composite with super high electrochemical and capacitance performances. Electrochim Acta. 2013;95:14654.

[221] Dong XY, Wang L, Wang D, Li C, Jin J. Layer-by-Layer Engineered Co-Al Hydroxide Nanosheets/Graphene Multilayer Films as Flexible Electrode for Supercapacitor. Langmuir. 2012;28:293-8.

[222] Zhang LJ, Zhang XG, Shen LF, Gao B, Hao L, Lu XJ, et al. Enhanced high-current capacitive behavior of graphene/CoAl-layered double hydroxide composites as electrode material for supercapacitors. J Power Sources. 2012;199:395-401.

[223] Yu X, Kang Y, Park HS. Sulfur and phosphorus co-doping of hierarchically porous graphene aerogels for enhancing supercapacitor performance. Carbon. 2016;101:49-56. 
[224] Dou S, Huang X, Ma Z, Wu J, Wang S. A simple approach to the synthesis of BCN graphene with high capacitance. Nanotechnol. 2015;26:045402.

[225] Yu X, Park SK, Yeon SH, Park HS. Three-dimensional, sulfur-incorporated graphene aerogels for the enhanced performances of pseudocapacitive electrodes. J Power Sources. 2015;278:484-9.

[226] Huang JZ, Yao NN, Deng XL, Fu K, Chen SY. Enhanced Efficiency of Dye-Sensitized Solar Cells Benefited from Graphene Modified by Ag Nanoparticles. J Nanosci Nanotechnol. 2018;18:3693-6.

[227] Wei XJ, Li D, Jiang W, Gu ZM, Wang XJ, Zhang ZX, et al. 3D Printable Graphene Composite. Sci Rep. 2015;5:11181.

[228] Zhang D, Chi BH, Li BW, Gao ZW, Du Y, Guo JB, et al. Fabrication of highly conductive graphene flexible circuits by 3D printing. Synth Metals. 2016;217:79-86.

[229] Manzanares Palenzuela CL, Novotný F, Krupička P, Sofer Z, Pumera M. 3D-Printed Graphene/Polylactic Acid Electrodes Promise High Sensitivity in Electroanalysis. Anal Chem. 2018;90:5753-7.

[230] Garcia-Tunon E, Feilden E, Zheng H, D'Elia E, Leong A, Saiz E. Graphene Oxide: An All-in-One Processing Additive for 3D Printing. ACS Appl Mater Interfaces. 2017;9:3297789.

[231] Gnanasekaran K, Heijmans T, van Bennekom S, Woldhuis H, Wijnia S, de With G, et al. 3D printing of CNT-and graphene-based conductive polymer nanocomposites by fused deposition modeling. Appl Mater Today. 2017;9:21-8.

[232] Foo CY, Lim HN, Mahdi MA, Wahid MH, Huang NM. Three-Dimensional Printed Electrode and Its Novel Applications in Electronic Devices. Sci Rep. 2018;8:7399.

[233] Cardoso RM, Mendonca DMH, Silva WP, Silva MNT, Nossol E, da Silva RAB, et al. 3D printing for electroanalysis: From multiuse electrochemical cells to sensors. Anal Chim Acta. 2018;1033:49-57.

[234] Camargo JC, Machado AR, Almeida EC, Moura Sousa Silva EF. Mechanical properties of PLA-graphene filament for FDM 3D printing. Int J Adv Manufact Technol. 2019;103:2423-43.

[235] Lin D, Jin SY, Zhang F, Wang C, Wang YQ, Zhou C, et al. 3D stereolithography printing of graphene oxide reinforced complex architectures. Nanotechnol. 2015;26:404003.

[236] Zhang QQ, Zhang F, Xu X, Zhou C, Lin D. Three-Dimensional Printing Hollow Polymer Template-Mediated Graphene Lattices with Tailorable Architectures and Multifunctional Properties. ACS Nano. 2018;12:1096-106.

[237] Wang DY, Huang X, Li J, He B, Liu Q, Hu LG, et al. 3D printing of graphene-doped target for "matrix-free" laser desorption/ionization mass spectrometry. Chem Commun. 2018;54:2723-6.

[238] Zhou G, Wang KP, Liu HW, Wang L, Xiao XF, Dou DD, et al. Three-dimensional polylactic acid@graphene oxide/chitosan sponge bionic filter: Highly efficient adsorption of crystal violet dye. Int J Biol Macromol. 2018;113:792-803.

[239] Manapat JZ, Mangadlao JD, Tiu BDB, Tritchler GC, Advincula RC. High-Strength Stereolithographic 3D Printed Nanocomposites: Graphene Oxide Metastability. ACS Appl Mater Interfaces. 2017;9:10085-93.

[240] Chiappone A, Roppolo I, Naretto E, Fantino E, Calignano F, Sangermano M, et al. Study of graphene oxide-based 3D printable composites: Effect of the in situ reduction. Comp Part B-Eng. 2017;124:9-15.

[241] Korhonen H, Sinh LH, Luong ND, Lehtinen P, Verho T, Partanen J, et al. Fabrication of graphene-based 3D structures by stereolithography. Phys Status Solidi A. 2016;213:982-5.

[242] Huang K, Yang JS, Dong SM, Feng Q, Zhang XY, Ding YS, et al. Anisotropy of graphene scaffolds assembled by three-dimensional printing. Carbon. 2018;130:1-10. 
[243] Zhu C, Han TYJ, Duoss EB, Golobic AM, Kuntz JD, Spadaccini CM, et al. Highly compressible 3D periodic graphene aerogel microlattices. Nat Commun. 2015;6:6962.

[244] Zhang QQ, Zhang F, Medarametla SP, Li H, Zhou C, Lin D. 3D Printing of Graphene Aerogels. Small. 2016;12:1702-8.

[245] Ma JH, Wang P, Dong L, Ruan YB, Lu HB. Highly conductive, mechanically strong graphene monolith assembled by three-dimensional printing of large graphene oxide. $\mathrm{J}$ Colloid Interf Sci. 2019;534:12-9.

[246] Lacey SD, Kirsch DJ, Li YJ, Morgenstern JT, Zarket BC, Yao YG, et al. ExtrusionBased 3D Printing of Hierarchically Porous Advanced Battery Electrodes. Adv Mater. 2018;30:1705651.

[247] Yao B, Chandrasekaran S, Zhang J, Xiao W, Qian F, Zhu C, et al. Efficient 3D Printed Pseudocapacitive Electrodes with Ultrahigh $\mathrm{MnO}_{2}$ Loading. Joule. 2019;3:459-70.

[248] Wang T, Li L, Tian X, Jin H, Tang K, Hou S, et al. 3D-printed interdigitated graphene framework as superior support of metal oxide nanostructures for remarkable micropseudocapacitors. Electrochim Acta. 2019;319:245-52.

[249] Truby RL, Lewis JA. Printing soft matter in three dimensions. Nature. 2016;540:371-8. [250] Tumbleston JR, Shirvanyants D, Ermoshkin N, Janusziewicz R, Johnson AR, Kelly D, et al. Continuous liquid interface production of 3D objects. Science. 2015;347:1349-52. 
Table captions:

Table 1. List of the M/graphene-based products produced by chemical reduction with corresponding reducing agent and surface properties.

Table 2. List of the monometallic Pt loaded graphene based electrocatalysts in MOR and EOR.

Table 3. List of common monometallic non-Pt and bimetallic graphene based electrocatalysts and their structure.

Table 4. List of the monometallic non-Pt and bimetallic graphene based electrocatalyst performance in MOR, EOR, FOR and GOR.

Table 5. List of graphene-based LIBs, Li-Air battery electrode activity.

Table 6. List of graphene-based materials for supercapacitor electrode.

Table 7. List of graphene-based supercapacitor electrode performance.

Table 8. List of best performance of graphene based DSSC.

Table 9. List of green energy research materials involving the use of 3D printed GA based materials against traditional GA based products.

Table 10. Advantage and disadvantage of existing 3D printing method for graphene based green energy applications.

Figure captions:

Fig 1. a) Photograph of a reduced GO wet gel before drying (left), after supercritical drying (middle), and after ambient drying (right). For reference, the wet gel is in a $20 \mathrm{ml}$ vial. Inset shows a GDC cylinder (cast), prism (machined), and pyramid (machined). SEM images of fracture surfaces of $b, c$ ) the GMA and d,e) GDC at b,d) low and c,e) high magnification. f) TEM images of commercial graphite at high magnification. Inset is a zoom-in of white box area and is $10 \mathrm{~nm}$ in width. g) TEM image of GDC at high magnification. Inset is a zoom-in of white box area and is $10 \mathrm{~nm}$ in width. h) Low magnification TEM image of GDC.[64] (Wiley Interscience)

Fig 2. a) Fabrication of GHCs and GHC sandwiches; Optical images of b-d) GHCs with different GA wall thicknesses, $t$, ranging from 0.25 to $1 \mathrm{~mm}$, and $\mathrm{e}-\mathrm{g}$ ) the corresponding GHC/PDMS composites; SEM images of h) GHC and i) GHC/PDMS composites.[66] (Wiley Interscience) 
Fig 3. SEM images of (a) NF, (b, c) as-prepared GOA@NF at different magnifications, and (d-f) as-prepared GA@NF at diff erent magnifications.[33] (ACS)

Fig 4. Photographs of (a) GO aqueous dispersions, (b) GO hydrogel with the insert of a NF sheet, and (c) NF, GOA@NF, and GA@NF.[33] (ACS)

Fig 5. (a) HRTEM and (b) TEM images (inset, size-distribution histogram) of PtNCs in NGA; (c) SEM image (inset, photograph of bulk PtNCs@NGA) and (d) magnified SEM image of PtNCs@NGA.[73] (RSC)

Fig 6. Low and high magnificent SEM images of (a) and (b) of GAs, and (c) and (d) of GA/Pd NP hybrids. (e) Dark-field TEM and HR-TEM images of GA/Pd NP hybrids. Inset of SAED pattern of GA/Pd NP hybrids.[122] (Elsevier B. V.)

Fig 7. (a, b) Low-magnification STEM images of $2.1 \mathrm{~nm}$ thick Pt nanoclusters coated GNFs. Inset is the corresponding EDS spectrum taken in SEM. High-resolution STEM (c, enlarged image of the marked square area of (b)) and HAADF (d) images of $2.1 \mathrm{~nm} \mathrm{Pt}$ nanoclusters on GNFs, revealing 2-4 nm monolayer Pt nanoclusters well intercoupled on both basal and edge planes of high-quality GNFs.[20] (ACS)

Fig 8. (a) Lower- and (b) higher-magnification SEM images of as-electrodeposited Pd on the rGO/CFP electrode, (c) HRTEM image, and (d) electron diffraction pattern of aselectrodeposited Pd.[47] (RSC)

Fig 9. TEM images of FS-Pd/GO composites in different scales. The inset of (A) is the sizehistogram of FS-Pd.[118] (Elsevier B. V.)

Fig 10. (a) CV curves of GM-Co-B-N in $\mathrm{O}_{2}$ - and $\mathrm{N}_{2}$-saturated electrolyte. (b) Polarization curves of G-Co, G-Co-B, G-Co-N, G-Co-B-N, and GM-Co-B-N at 1600 rpm rotation speed in $\mathrm{O}_{2}$-saturated electrolyte. (c) LSV curves of GM-Co-B-N in diff erent rotation speeds in the range from 400 to $2500 \mathrm{rpm}$, inset shows the K-L plot of GM-Co-B-N. (d) Electron transfer numbers of catalyst samples. (e) The durability of electrodes and (f) current density loss-time CA responses of GM-Co-B-N and Pt/C electrodes at -0.3 $\mathrm{V}$ in oxygen-saturated electrolyte at rotating speed of $1200 \mathrm{rpm}$. The arrow indicates the addition of $3 \mathrm{M}$ methanol into the electrochemical cells.[7] (ACS)

Fig 11. (a) Chronoamperometric responses of the PtNCs@NGA and commercial Pt/C catalysts for $12 \mathrm{~h}$ in an $\mathrm{O}_{2}$-saturated aqueous solution of $0.5 \mathrm{M} \mathrm{H}_{2} \mathrm{SO}_{4}$. (b) Chronoamperometric responses of the PtNCs@NGA and Pt/C catalysts in an $\mathrm{O}_{2}$ saturated aqueous solution of $0.5 \mathrm{M} \mathrm{H}_{2} \mathrm{SO}_{4}$ to injection of $1 \mathrm{M}$ methanol.[73] (RSC) 
Fig 12. CVs of Pd-Cu NCs/RGOs (A), RGOs (B), and Pd black (C) modified electrodes without (curve a) and with (curve b) $3.0 \mathrm{M}$ methanol in $0.1 \mathrm{M} \mathrm{KOH}$ at the scan rate of $50 \mathrm{mV} \mathrm{s}^{-1} \cdot[105]$ (Elsevier B. V.)

Fig 13.(a) Open-circuit voltage of GM-Co-B-N-based Zn-air battery. (b) Typical galvanostatic discharge curves of $\mathrm{Zn}$-air batteries with GM-Co-B-N and $\mathrm{Pt} / \mathrm{C}$ as cathode catalysts at $10 \mathrm{~mA} \mathrm{~cm}$-2 current densities. (c) Long-term galvanostatic discharge curves of Zn-air batteries and (d) galvanostatic discharge-charge cycling curves, current density $10 \mathrm{~mA} \mathrm{~cm}{ }^{-2}, 20$ min for each state.[7] (ACS)

Fig 14. First three charge/discharge curves of GA (a) and N-GA (b) at $100 \mathrm{~mA} \mathrm{~g}^{-1}$; Cycling performances of GA and N-GA at $100 \mathrm{~mA} \mathrm{~g}^{-1}$ (c) and $500 \mathrm{~mA} \mathrm{~g}^{-1}$ (d); Rate capability at different current density (e) and Nyquist plots (f) of GA and N-GA.[9] (Elsevier B. V.)

Fig 15. (A) $\mathrm{CV}$ curves of $\mathrm{AC}, \mathrm{GA}, \mathrm{GA} / \mathrm{TiO}_{2}$ in $0.1 \mathrm{M} \mathrm{NaCl}$ solution at a scan rate of $100 \mathrm{mV}$ $\mathrm{s}^{-1}$; (B) The specific capacitance of $\mathrm{AC}, \mathrm{GA}, \mathrm{GA} / \mathrm{TiO}_{2}$ at different scan rates; (C) Cycling performance of $\mathrm{GA} / \mathrm{TiO}_{2}$ at a scan rate of $100 \mathrm{mV} \mathrm{s}^{-1}$, inset presents the $\mathrm{CV}$ curves before and after the 1000 cycles. (D) Desalination capacity of AC, GA and $\mathrm{GA} / \mathrm{TiO}_{2}$ with different equilibrium concentrations and the corresponding Langmuir isotherm fitted curves; (E) Desalination efficiency of $\mathrm{AC}$, GA and $\mathrm{GA} / \mathrm{TiO}_{2}$ in 500 $\mathrm{mg} / \mathrm{L} \mathrm{NaCl}$ solution at different times; (F) Electrosorption and regeneration cycles of $\mathrm{GA} / \mathrm{TiO}_{2}$ in $500 \mathrm{mg} / \mathrm{L} \mathrm{NaCl}$.[134] (Wiley Interscience)

Fig 16. (a) Schematics of ZGR based DSSC (b) Typical energy levels of various components of ZGR based DSSC employing an iodide/triiodide based redox electrolyte and N749 as a sensitizer.[199] (Springer)

Fig 17. J-V curves of DSSCs based on (a) ZGR 0.25 (b) ZGR 0.5 (c) ZGR 1 and (d) ZGR 0 nanocomposite films.[199] (Springer)

Fig 18. Electron transfer mechanism in $\mathrm{ZnO}$ nanoparticles and graphene/ZnO nanocomposite on irradiation.[200] (Elsevier BV)

Fig 19. Mechanism of hydrogen production in the $\mathrm{GO}^{-\mathrm{TiO}_{2}}$ composite system.[139] (Wiley Interscience)

Fig 20. Common light- and ink-based 3D printing methods. a, The light-based 3D printing method known as continuous liquid interface production. (Diagram adapted from [250]) b, Light-based selective laser sintering of powders. c, Light- and ink-based photocurable inkjet printing of photopolymerizable resins. $d$, Ink-based fused deposition modelling of thermoplastic filaments. e, Direct ink writing using 
viscoelastic inks [249] (Nature group). f, Fabrication illustration of BN-GAs hydrothermal process and freeze-drying process. g, Digital images of two pairs of GO solutions with different volumes in the vials and N/B co-doped graphene hydrogels before and after hydrothermal self-assembly. Inset: digital images of the BN-GAs obtained after freeze-drying [148] (Wiley Interscience).

Fig 21. (A) Tensile strength comparison of cast and 3D-printed parts. SLA-printed complexshaped GO nanocomposites: (B) nested dodecahedron and (C) diagrid ring.[239] $(\mathrm{ACS})$

Fig 22. Tensile strength as a function of GO loading.[239] (ACS)

Fig 23. Physiochemical characterization. (a) Optical image of the 3D printing process, (b) 3D printed electrode used throughout the study. (c) FESEM image of 3DE/Au electrode, and (d) corresponding magnified cross-sectional area.[232] (Nature group)

Fig 24. Morphology and structure of graphene aerogels. (a) Optical image of a 3D printed graphene aerogel microlattice. SEM images of (b) a 3D printed graphene aerogel microlattice, (c) graphene aerogel without R-F after etching and (d) graphene aerogel with $4 \mathrm{wt} \% \mathrm{R}-\mathrm{F}$ after etching. Optical image of (e) 3D printed graphene aerogel microlattices with varying thickness and (f) a 3D printed graphene aerogel honeycomb. Scale bars, 5mm (a), 200mm (b), 100nm (c, d), 1cm (f).[243] (Nature group)

Fig 25.3D printed GO aerogels. (a)-(c) Printed GO aerogels on catkin, and (d)-(f) designs of printed GO aerogels.[244] (Wiley Interscience)

Fig 26. (a) Experimental setup for compression by DMA. (b) Loading and unloading process during compression test (50\% strain).[244] (Wiley Interscience)

Fig 27.LGO ink properties and schematic diagram of the fabrication process (a) Digital image of the LGO ink with a concentration of $20 \mathrm{mg} / \mathrm{mL}$ in a $30 \mathrm{~cm}^{3}$ barrel, (b) Viscosity as a function of the shear rate for LGO ink, (c) G0 and G00 as a function of the shear stress for LGO ink, (d) the LGO ink extrusion through micro needle (400 $\mu \mathrm{m}$ ), (e) the model of printed 3D LGO monolith, (f) chemical reduction of LGO monolith by HI, (g) thermal annealing of monolith at $1000{ }^{\circ} \mathrm{C}$ in nitrogen, (h) the optical image of the final low-density 3D graphene monolith, (i) the printed 3D letter FDU, (j) the electrical property of T-LGO monolith, (k) the compressive and resilient property of T-LGO monolith.[245] (Elsevier B. V.)

Fig 28. The photograph of the printed 3D letter FDU, fork type and hexagon.[245] (Elsevier B. V.) 
Fig 29. Schematic of the coin cell fabrication (A), charge-discharge profiles (B), cycling properties (C), coulombic efficiency (D) and rate capability of the 3D printed anode (E).[10] (Nature group)

Fig 30. Cyclic voltammetry (A) of the 3D-SC consisting of a $2 \mathrm{~mm}$ layer of solid electrolyte of PVA and $1.0 \mathrm{M} \mathrm{H}_{2} \mathrm{SO}_{4}$. Corresponding charge/discharge curves with (C) and without (B) the Kampouris' circuit in parallel are also presented. Scan Rate: $25 \mathrm{mV}$ $\mathrm{s}^{-1}$. Inset to A is a schematic of the 3D-SC utilised throughout this study.[10] (Nature group)

Fig 31. Comparative linear sweep voltammograms (LSV) (A) using 3DE compared to EPPGE, GCE, BDDE and platinum showing the onset of the HER. Stability studies of the 3DEs (B) using LSV for the initial, 10th, 100th and 1000th scans. Scan rate: 25 $\mathrm{mV} \mathrm{s}^{-1}$ (vs. SCE). Note: $3 \mathrm{DEN}=1$ is upon the initial scan and $3 \mathrm{DEN}=1000$ is upon the 1000th scan.[10] (Nature group)

Fig 32. Electrochemical performance of the coin-type symmetric microsupercapacitors. (a) $\mathrm{CV}$ curves collected at scan rates of $20-100 \mathrm{mV} / \mathrm{s}$ in $6 \mathrm{M} \mathrm{KOH}$ electrolyte. (b) GCD profiles collected at current densities from 4 to $40 \mathrm{~mA} \mathrm{~cm}^{-2}$. (c) Areal capacitance and capacitive retention versus diff erent areal current densities $\left(4-40 \mathrm{~mA} \mathrm{~cm}^{-2}\right)$. The inset shows optical images of the device before and after assembling. (d) Cycling stability measurements. The inset shows an optical image of a green LED-lighted by three coins connected in series.[12] (ACS) 